Aus dem Institut für Epidemiologie (Ärztliche Leitung Prof. Dr. Wolfgang Lieb)

im Universitätsklinikum Schleswig-Holstein, Campus Kiel an der Christian-Albrechts-Universität zu Kiel

\title{
Direkte und indirekte Kosten einer Geburt bei Insulanerinnen, wenn vor Ort keine Geburtshilfe möglich ist
}

\author{
Inauguraldissertation \\ zur Erlangung \\ der Doktorwürde der Medizin \\ der Medizinischen Fakultät \\ der Christian-Albrechts-Universität zu Kiel \\ vorgelegt von \\ Jill Charlotte Jaursch \\ aus Düsseldorf
}

Kiel 2019 
1. Berichterstatter: Prof. Dr. med. Roland Diel, Institut für Epidemiologie

2. Berichterstatter: Priv.-Doz. Dr. med. André Farrokh, Klinik für Gynäkologie und Geburtshilfe Tag der mündlichen Prüfung: 09. Oktober 2020

Zum Druck genehmigt, Kiel, den 15. Juli 2020

gez.: Prof. Dr. Johann Roider

(Vorsitzender der Prüfungskommission) 


\section{Inhaltsverzeichnis}

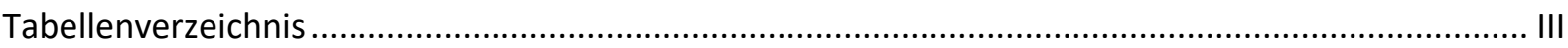

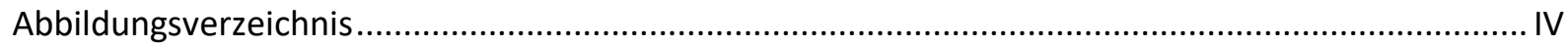

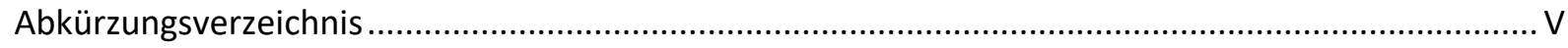

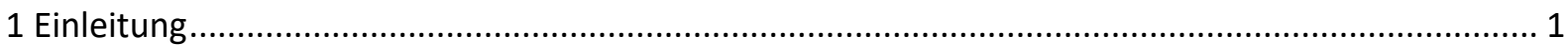

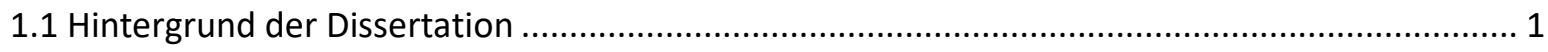

1.1.1 Geburtshilfliche Situation in Deutschland mit Fokus auf das Bundesland Schleswig-

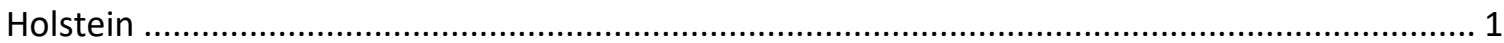

1.1.2 Geburtshilfliche Situation auf Föhr vor und nach Schließung der stationären Geburtshilfe. 8

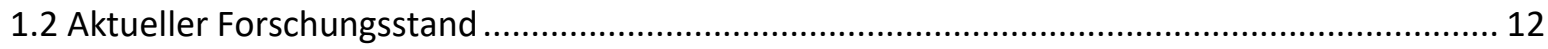

1.3 Fragestellung und Zielsetzung der vorliegenden Arbeit ........................................................ 14

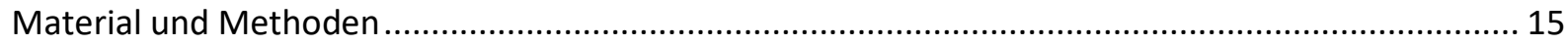

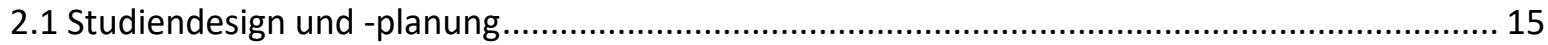

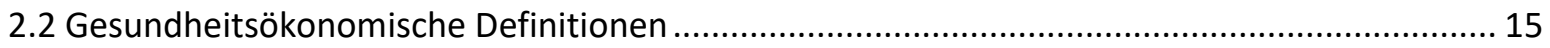

2.2.1 Identifikation relevanter Kostenkomponenten .............................................................. 16

2.2.2 Direkte medizinische und direkte nicht-medizinische Kosten ......................................... 18

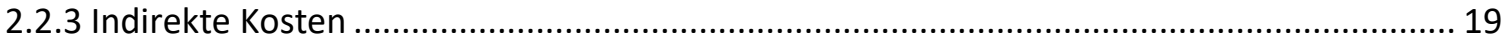

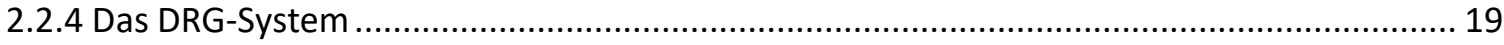

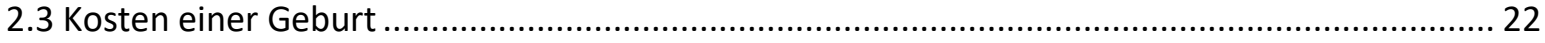

2.3.1 Fallzahlen Klinikum Nordfriesland gGmbH, Standort Wyk/ Föhr ......................................... 26

2.3.2 Erfassung der Kosten einer Geburt in einer Belegabteilung, in einer Hauptabteilung mit Beleghebammen sowie einer Hauptabteilung mit angestellten Hebammen ............................... 29

2.3.3 Messung der Ressourcenverbräuche und monetäre Bewertung der Ressourcenverbräuche

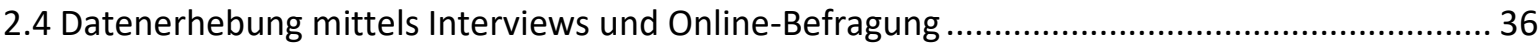

2.4.1 Vorarbeiten und Durchführung der qualitativen Interviews ............................................. 36

2.4.2 Rekrutierung und Einschlusskriterien der Teilnehmerinnen der Interviews ....................... 36

2.4.4 Auswertung und Ergebnisse der qualitativen Interviews ................................................ 37

2.4.5 Erstellen der Online-Umfrage und Einschlusskriterien ...................................................... 39

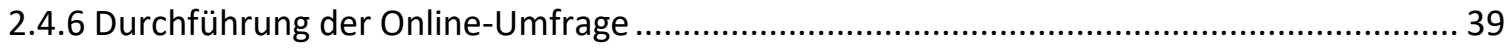

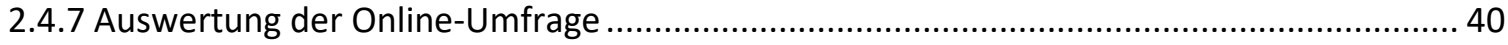

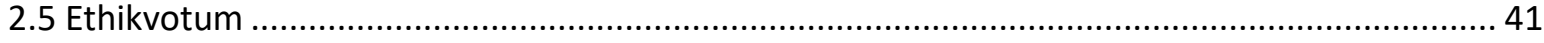

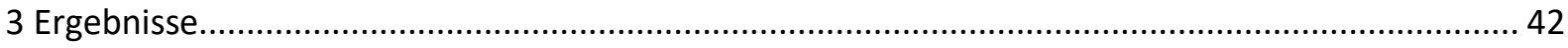




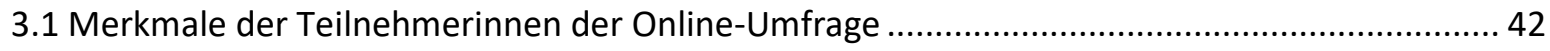

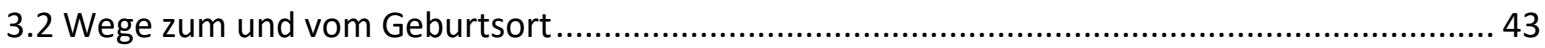

3.3 Art, Ort der Unterkunft, Verweildauern auf dem Festland und Geburtsmodi ........................... 44

3.4 Aufstellung der Kosten im Rahmen der Online-Umfrage ..................................................... 48

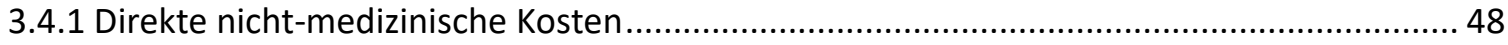

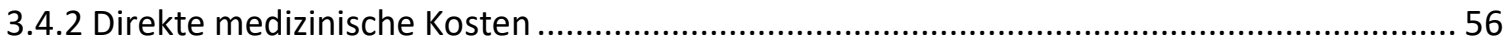

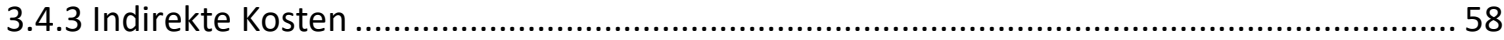

3.5 Aufstellung der Gesamtkosten einer Geburt vor und nach Schließung der stationären

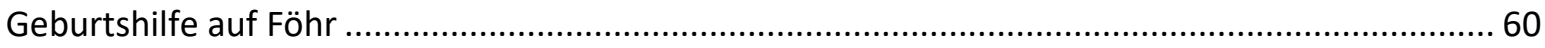

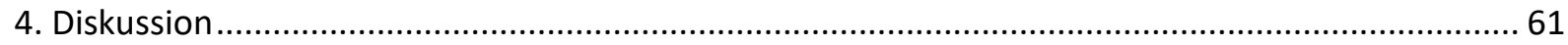

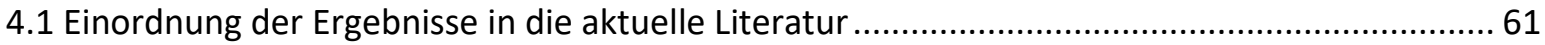

4.2 Einordung der Ergebnisse in die aktuelle gesundheitspolitische Debatte ................................ 65

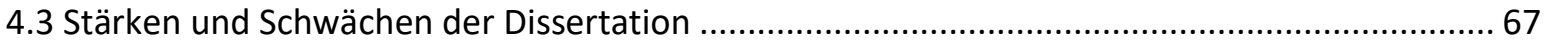

5. Zusammenfassung mit Schlussfolgerungen und Empfehlungen ................................................... 68

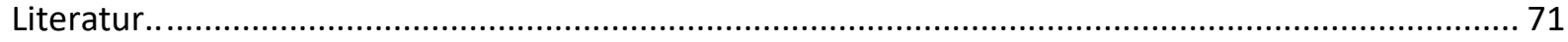

Anhang

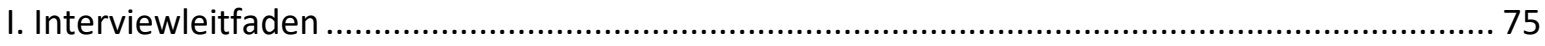

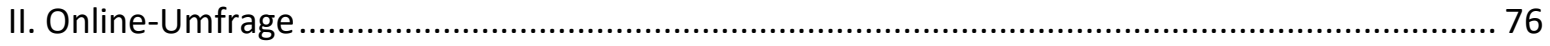

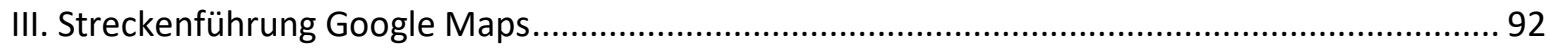

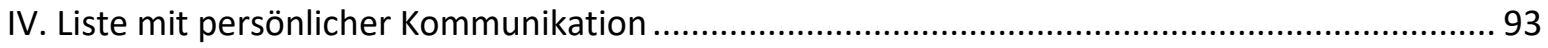

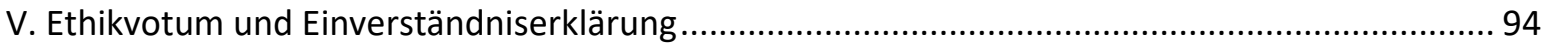

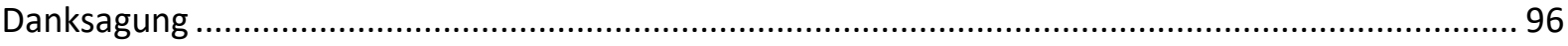

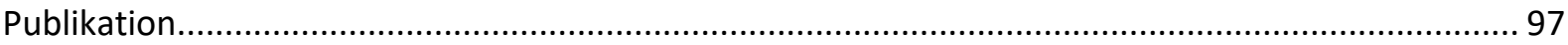




\section{Tabellenverzeichnis}

Tabelle 1: Geburtskliniken in Schleswig-Holstein. Quelle: www.g-ba.de ........................................... 6

Tabelle 2: Aufbau einer DRG als Beispiel im Rahmen der Geburtshilfe .............................................. 20

Tabelle 3: Bewertungsrelationen der DRGs aus den Fallpauschalenkatalogen und

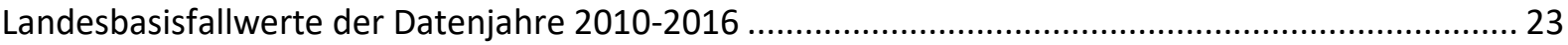

Tabelle 4: Abrechnung einer Geburt nach DRG Entgeltrechnung in Euro ........................................... 24

Tabelle 5: Abrechnung einer Geburt in Euro nach dem einheitlichen Bewertungsmaßstab (EBM) der

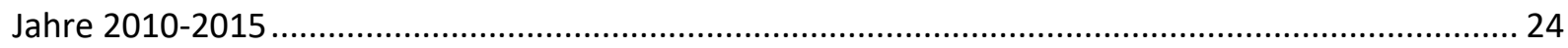

Tabelle 6: Abrechnung einer Geburt in Euro nach der Hebammen-Vergütungsvereinbarung ............ 25

Tabelle 7: Fallzahlen Klinikum Nordfriesland gGmbH, Inselklinik Föhr/ Amrum ................................ 26

Tabelle 8: Fallzahlen der geburtshilflichen DRGs in der Inselklinik Föhr/Amrum 2010-2015. Quelle: Qualitätsreporte Klinikum Nordfriesland gGmbH, Inselklinik Föhr/Amrum 2010-2015..................... 26

Tabelle 9: Fallzahlen der geburtshilflichen DRGs aus persönlicher Quelle einer Föhrer Hebamme. Quelle: Persönliche Angaben einer Föhrer Hebamme.

Tabelle 10: Geburten im Krankenhaus "Inselklinik Föhr/Amrum", entnommen aus der Drucksache 13-

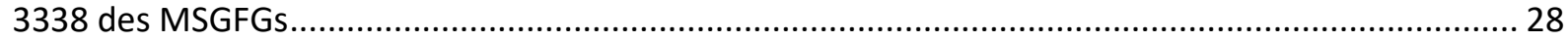

Tabelle 11: Kosten einer Geburt in Euro in einer Belegabteilung ..................................................... 29

Tabelle 12: Kosten einer Geburt in Euro in einer Hauptabteilung mit Beleghebammen ...................... 30

Tabelle 13: Kosten einer Geburt in Euro in einer Hauptabteilung ...................................................... 30

Tabelle 14: Minimale Transportkosten einer Geburt auf dem Festland, wenn die Eltern Föhrer sind 32

Tabelle 15: Verbrauch und monetäre Bewertung der Ressourcen ...................................................... 35

Tabelle 16: Charakteristika der Teilnehmerinnen an der Online-Umfrage ........................................... 42

Tabelle 17: Direkte nicht- medizinische Kosten der Teilnehmerinnen in Euro ................................... 49

Tabelle 18: Zusammenfassung der direkten nicht-medizinischen Kosten in Abhängigkeit der gewählten Unterkunft in Euro $(n=29)$

Tabelle 19: Gesamte direkte nicht-medizinische Kosten der Leistungsträger nach Schließung der

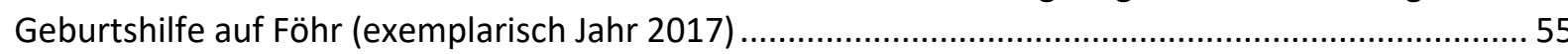

Tabelle 20: Gesamte direkte nicht- medizinische Kosten pro Jahr vor Schließung der Geburtshilfe auf Föhr

Tabelle 21: Direkte medizinischen Kosten in Euro der stationären Geburtshilfe auf Föhr.................... 57

Tabelle 22: Direkte medizinische Kosten in Euro bei Entbindung auf dem Festland............................ 58

Tabelle 23: Indirekte Kosten der Teilnehmerinnen in Euro $(n=8)$................................................... 58

Tabelle 24: Aufstellung der Gesamtkosten einer Geburt vor und nach Schließung der stationären Geburtshilfe auf Föhr... 


\section{Abbildungsverzeichnis}

Abbildung 1: Geburtsabteilungen in Schleswig-Holstein 1991-2016. Quelle: www.gbe-bund.de ......... 2

Abbildung 2: Lebendgeburten pro Jahr in Schleswig-Holstein 1991-2016. Quelle: Statistisches

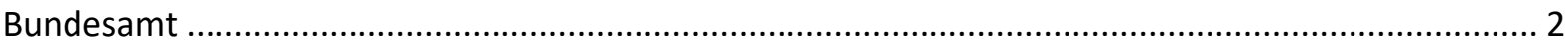

Abbildung 3: Chronologie der Schließung der Geburtshilfe auf Föhr ............................................... 11

Abbildung 4: Kostenkategorien im Rahmen der Studie ................................................................. 17

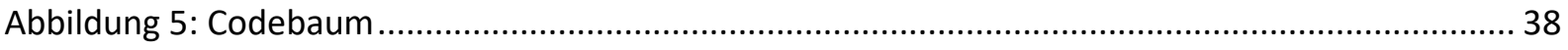

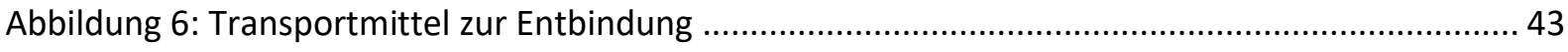

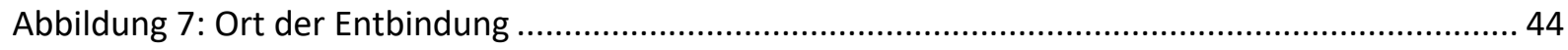

Abbildung 8: Unterkunftsorte der Teilnehmerinnen auf dem Festland vor der Geburt ...................... 45

Abbildung 9: Dauer des prästationären Aufenthaltes der Teilnehmerinnen auf dem Festland $(n=24) 46$

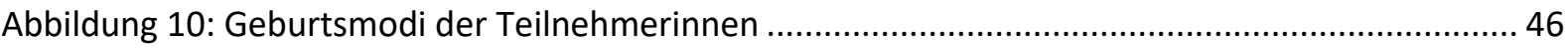

Abbildung 11: Stationäre Verweildauern der Teilnehmerinnen ....................................................... 47

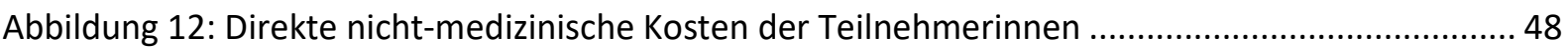

Abbildung 13: Verpflegungskosten der Teilnehmerinnen aufgeschlüsselt nach Unterkunftsart $(n=29)$

Abbildung 14: Fahrtkosten der Teilnehmerinnen aufgeschlüsselt nach Unterkunftsart $(n=29) \ldots \ldots . . . .50$

Abbildung 15: Fähr- und Parkkosten der Teilnehmerinnen aufgeschlüsselt nach Unterkunftsart ( $n=29)$

Abbildung 16: Unterkunftskosten der Teilnehmerinnen aufgeschlüsselt nach Unterkunftsart $(n=28)^{*}$

Abbildung 17: Sonstige Kosten der Teilnehmerinnen aufgeschlüsselt nach Unterkunftsart $(n=29) \ldots 52$

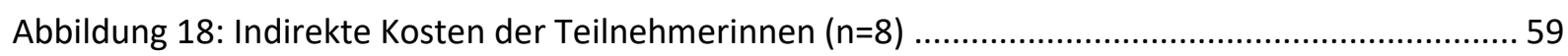




\section{Abkürzungsverzeichnis}

AOK Allgemeine Ortskrankenkasse

AWMF. .Arbeitsgemeinschaft der Wissenschaftlichen Medizinischen Fachgesellschaften

$\mathrm{CCL}$ Complication or Comorbidity Level

DGGG Deutsche Gesellschaft für Gynäkologie und Geburtshilfe

DGHWi Deutsche Gesellschaft für Hebammenwissenschaft DGPM Deutsche Gesellschaft für Perinatale Medizin

DGzRS Deutsche Gesellschaft zur Rettung Schiffbrüchiger

DKG Deutsche Krankenhausgesellschaft DRF .Deutsche Rettungsflugwacht. DRG Diagnosis Related Groups

e.V. eingetragener Verein EBM Einheitlicher Bewertungsmaßstab

E-E-Zeit Entscheidungs-Entbindungszeit

GBA ...Gemeinsamer Bundesauschuss

GBE Gesundheitsberichterstattung des Bundes GKV Gesetzliche Krankenversicherung

ICD International Classification of Diseases InEK Institut für Entgeltsystem

IQR Interquartilsabstand KBV Kassenärztliche Bundesvereinigung MDC Major Diagnostic Category OPS Operations-und Prozedurenschlüssel PCCL Patient Clinical Complexity Level PKV Private Krankenversicherungen SD. Standardabweichung UKSH Universitätsklinikum Schleswig-Holstein 


\section{Einleitung}

\subsection{Hintergrund der Dissertation}

Ausgangspunkt dieser Forschungsarbeit ist die Schließung der geburtshilflichen Abteilung auf der nordfriesischen Insel Föhr in Deutschland zum 1. Oktober 2015. Die Schließung dieser Abteilung steht im Kontext einer größeren politischen Ausrichtung, in der es um die Zentralisierung und Spezialisierung im Gesundheitswesen in Deutschland geht. In wohl keinem anderen medizinischen Fach wird die Debatte um Abteilungsschließungen von Fachleuten und der Öffentlichkeit so leidenschaftlich geführt wie in der Geburtshilfe, wo es gleich um das Wohl von zwei Menschen geht.

Diese Einleitung gibt einen Überblick über die geburtshilfliche Versorgungssituation in Deutschland, in Schleswig-Holstein und auf Föhr mit einem Schwerpunkt auf der Zentralisierung und Spezialisierung der Geburtshilfe. Weiterhin wird der aktuelle Stand der Literatur beleuchtet und schlussendlich eine Zielsetzung der vorliegenden Arbeit definiert.

\subsubsection{Geburtshilfliche Situation in Deutschland mit Fokus auf das Bundesland Schleswig- Holstein}

Die Zentralisierung und Spezialisierung in der Geburtshilfe der vergangenen Jahre lässt sich an folgenden Statistiken ablesen. Nimmt man eine Gliederung der Krankenhäuser nach ihrer Bettenzahl vor, so zeigt sich im Jahr 1991 für Kliniken mit bis zu 50 Betten noch eine Lebendgeburtenzahl von 14.979 Lebendgeburten, während es 2016 nur noch 2.426 Lebendgeburten waren. Das ist ein Rückgang von knapp 84 Prozent. Bei Krankenhäusern mit einer Bettenzahl von 50 bis 100 wurde im gleichen Zeitraum ein Rückgang der Lebendgeburten um 76,2 Prozent beobachtet und bei Krankenhäusern mit einer Bettenzahl von 100 bis 150 immerhin noch ein Rückgang der Lebendgeburten um 38,8 Prozent. Im Gegensatz dazu verzeichneten größere Krankenhäuser einen Geburtenzuwachs. Krankenhäuser mit einer Bettenanzahl von 600 bis 800 hatten im Vergleich zu 1991 im Jahre 201617 Prozent mehr Lebendgeburten und Krankenhäuser mit einer Bettenanzahl von 800 und mehr verzeichneten einen Lebendgeburtenzuwachs von 42,2 Prozent. [1]

Diese Entwicklungen sind auch an einem Rückgang der Entbindungsstationen zu beobachten. 1991 gab es in Deutschland noch 1.186 Krankenhäuser mit einer angeschlossenen Geburtsabteilung. 2016 betrug diese Zahl nur noch 690 [2]. Das ist ein Rückgang um mehr als 40 Prozent. In Schleswig-Holstein ist diese Entwicklung ebenso prägnant zu erkennen (Abbildung 1): Gab es 1991 noch 36 Geburtsabteilungen, so waren es 2016 nur noch 20, dies entspricht einem Rückgang von 44,44 Prozent [2]. Allein zwischen 2014 und 2016 haben vier Geburtsabteilungen in Schleswig-Holstein ihre Türen schließen müssen: Oldenburg in Holstein, Sylt, Föhr und Niebüll. 


\section{Krankenhäuser mit Geburtsabteilung in \\ Schleswig-Holstein}

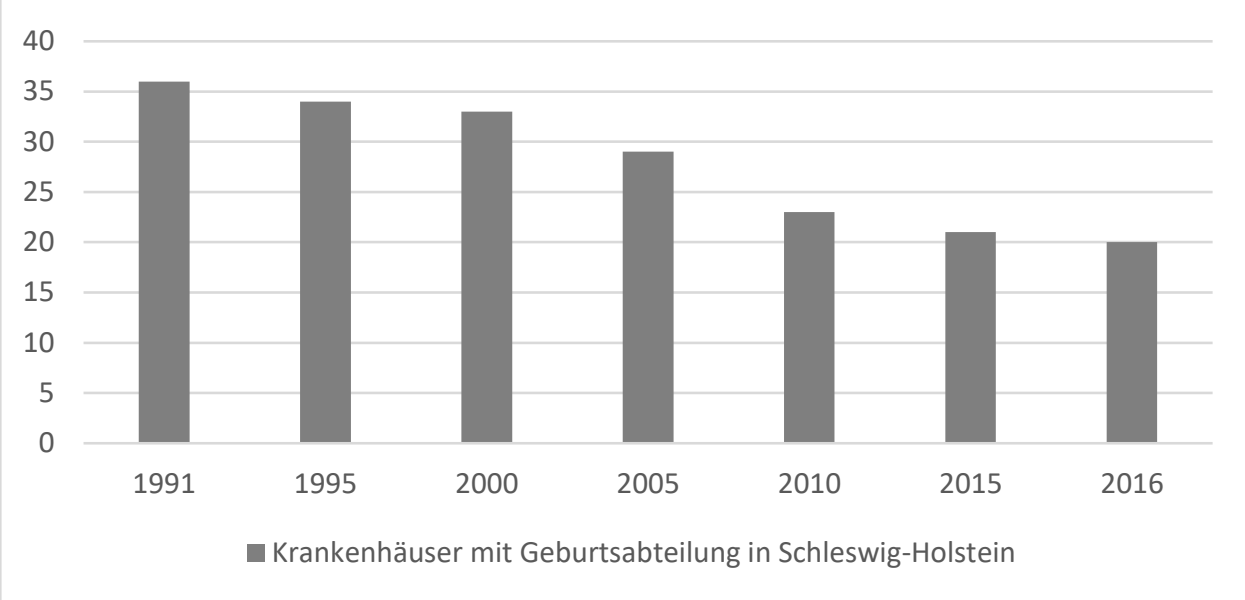

Abbildung 2: Lebendgeburten pro Jahr in Schleswig-Holstein 1991-2016. Quelle: Statistisches Bundesamt

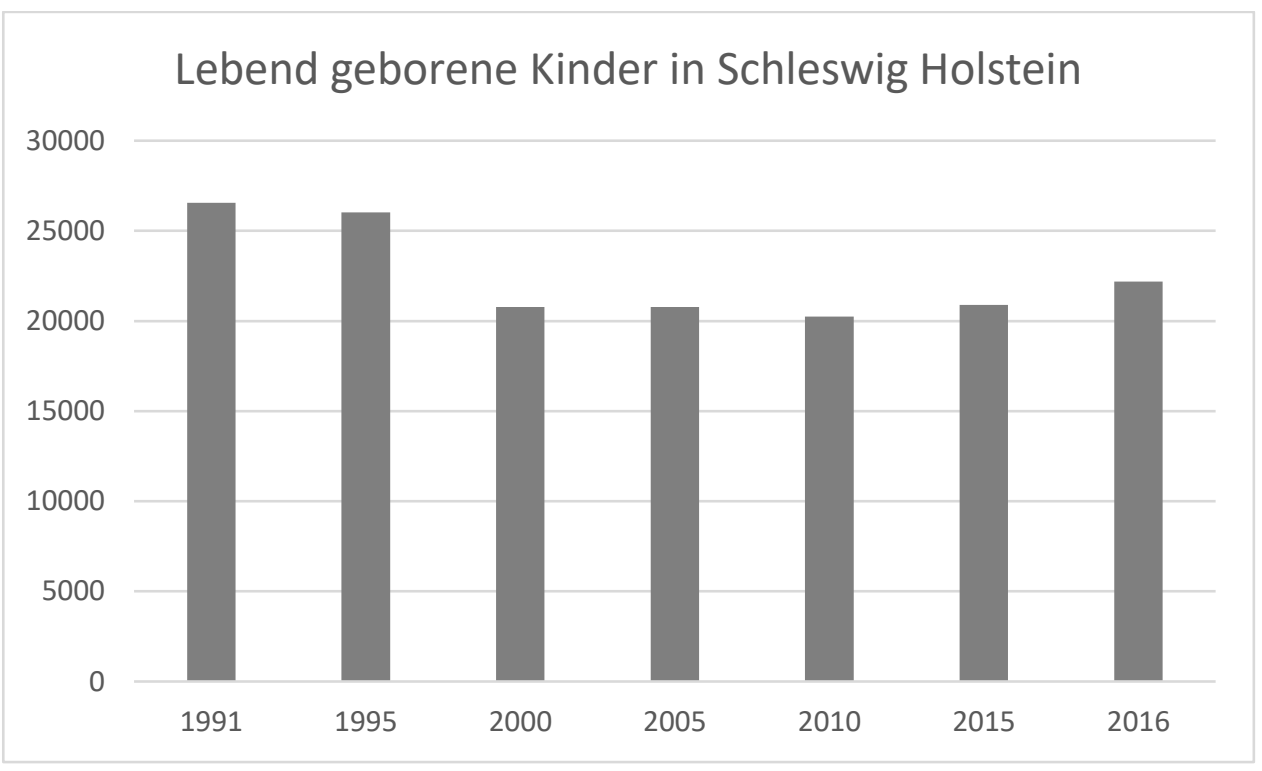

Die Gründe für die Zentralisierung und damit auch die Schließungen von Geburtshilfeabteilungen sind vielfältig. Die drei wichtigsten Gründe werden im Folgenden kurz beleuchtet. Alle drei hier aufgeführten Gründe können nicht separat voneinander evaluiert werden, sondern sind abhängig voneinander und in einem Zusammenhang zu betrachten. 


\section{1) Demographischer Wandel}

Während in Deutschland 1991 noch 830.351 Kinder lebend geboren wurden, waren es 2016 nur noch 774.464 Kinder [2]. Das entspricht einem Rückgang der Geburtenrate um 6,7 Prozent. Den Tiefpunkt erreichten die Zahlen 2011 mit 652.210 lebend geborenen Kindern in Deutschland. Das Statistische Bundesamt sagt in seiner 13. Koordinierten Bevölkerungsvorausberechnung voraus, dass die Geburtenzahl bis 2020 konstant bei 700.000 Neugeborenen pro Jahr bleiben wird. Bis zum Jahr 2060 wird die jährliche Geburtenzahl nur noch 500.000 bis 550.000 betragen. Die Geburtenziffer bleibt zunächst gleich bei 1,4 Kindern pro Frau bei der Annahme der annähernden Konstanz. Die annähernde Konstanz ist eine Annahme des statistischen Bundesamtes, die besagt, dass sich die Trends der Geburtenentwicklung ähnlich verhalten werden wie in den letzten Jahrzehnten. Allerdings erhöht sich der Anteil der Frauen, die in einem Alter von 30 oder später Kinder bekommen. Da statistisch gesehen Frauen, die ihr erstes Kind nach ihrem 30. Lebensjahr bekommen, weniger Kinder zur Welt bringen als Frauen, die vor 30 ihr erstes Kind bekommen, wird die Zahl der kinderreichen Mütter sinken. [3]

Somit kann man nicht davon ausgehen, dass es in Zukunft wieder zu einem Niveau der Geburtenrate von vor 20 Jahren kommt. Vor allem der ländliche Raum ist von dieser Entwicklung betroffen. In ländlichen Kreisen, wie zum Beispiel Ostholstein oder Nordfriesland in Schleswig-Holstein, ist die Geburtenzahl zwischen 1990 und 2015 um 31 Prozent gesunken, wohingegen in Städten wie Kiel die Geburtenzahl im gleichen Zeitraum nur um 9 Prozent gesunken ist [4].

\section{2) Qualitätssicherung}

Nicht nur der demographische Wandel trägt zu einer Zentralisierung der Geburtshilfe in Deutschland bei. Auch haben zunehmende Qualitätsanforderungen an die Geburtshilfe in den letzten Jahren dazu beigetragen, dass kleinere Geburtshilfeabteilungen geschlossen wurden.

In der Richtlinie des Gemeinsamen Bundesauschusses (GBA) über „die Maßnahmen zur Qualitätssicherung der Versorgung von Früh- und Reifgeborenen gemäß $\S 137$ Abs. 1 Nr. 2 SGB V in Verbindung mit $\S 92$ Abs. 1 Satz 2 Nr. 13 SGB V“ werden seit 2006 geburtshiffliche Einrichtungen in vier Kategorien eingeteilt. Diese Kategorien haben zum Ziel, konkrete Zuweisungs- und Aufnahmekriterien von Schwangeren in ein risikoadaptiertes Krankenhaus zu definieren sowie für eine Struktur-, Prozessund Ergebnisqualität der Geburtshilfe zu sorgen [5]. Die Kategorien sind folgende:

- Versorgungsstufe 1: Perinatalzentrum Level 1

- Versorgungsstufe 2: Perinatalzentrum Level 2

- Versorgungsstufe 3: Perinataler Schwerpunkt: Geburtshilfliche Abteilung mit angeschlossener Kinderklinik 
- Versorgungsstufe 4: Geburtsklinik: Geburtshilfliche Abteilung ohne angeschlossene Kinderklinik

Kliniken der Versorgungsstufen 1 bis 3 müssen Vorgaben des GBAs zur Struktur-, Prozess- und Ergebnisqualität erfüllen. Diese umfassen zum Beispiel Anforderungen zur ärztlichen und pflegerischen Versorgung in der Neonatologie und der Geburtshilfe sowie zu infrastrukturellen Anforderungen. Das Perinatalzentrum Level 2 darf zum Beispiel nur Frühgeborene mit einem geschätzten Geburtsgewicht von 1.250 Gramm bis 1.499 Gramm oder mit einem Gestationsalter von 29+0 bis 31+6 Schwangerschaftswochen aufnehmen. Frühgeborene, die leichter sind oder Schwangere, die in einer früheren Schwangerschaftswoche sind, werden im Perinatalzentrum Level 1 versorgt. Für die Versorgungsstufe 4 gibt es keine verbindlichen Vorschriften des GBAs; es wird lediglich darauf hingewiesen, dass die "Geburtsklinik (...) die Kriterien für eine Zuweisung in die höheren Versorgungsstufen im Rahmen ihres einrichtungsinternen Qualitätsmanagements als Prozessqualitätsmerkmal (beachtet).“ [5]

Für die Geburtskliniken der Versorgungsstufe 1, aber auch für alle anderen Versorgungsstufen, gelten daher die Leitlinien der Deutschen Gesellschaft für Gynäkologie und Geburtshilfe e.V. (DGGG). Die aktuelle S1 Leitlinie (015/078) der DGGG zu den „Mindestanforderungen an prozessuale, strukturelle und organisatorische Voraussetzungen für geburtshilfliche Abteilungen der Grund- und Regelversorgung" definiert zum Beispiel personelle Voraussetzungen für eine sichere Geburt, aber auch prozessuale Anforderungen wie eine Entscheidungs-Entbindungszeit (E-E-Zeit) von unter 20 Minuten (im Falle der Entscheidung zur Sectio) sowie ein adäquates Notfallmanagement mit einer sicheren Erstversorgung für Kind und Mutter. [6]

Weiterhin wurde im Mai 2015 eine überarbeitete Version der „Empfehlungen für die strukturellen Voraussetzungen der perinatologischen Versorgung in Deutschland“ von der Deutschen Gesellschaft für Perinatale Medizin e.V. (DGPM) veröffentlicht. Diese Empfehlungen ergänzen die Richtlinien des GBAs vor allem im Hinblick auf eine adäquate perinatologische Versorgung und thematisieren auch, „inwieweit isolierte Geburtskliniken ohne angeschlossene Kinderkliniken dem geforderten Aspekt einer maximalen Sicherheit für Mutter und Kind bei seltenen Komplikationen genügen, da insbesondere niedrige Geburtenzahlen (<500 p.a.) mit einer erhöhten neonatalen Letalität assoziiert sind." [7]

Die zitierten Richtlinien und Leitlinien bilden nicht die Gänze aller Vorschriften ab und sollen nur verdeutlichen, dass die Anforderungen an die Struktur-, Prozess- und Ergebnisqualität in den vergangenen Jahren erhöht wurden. Auch die Einführung von Mindestmengen für die Versorgung von Früh- und Neugeborenen für die Versorgungsstufen 1 und 2 im Jahre 2010 hebt hervor, dass die 
Überzeugung vorherrscht, dass eine durchgehende Sicherheit für Kind und Mutter nur in einer Geburtsabteilung mit entsprechenden Eingriffszahlen möglich ist: Je häufiger ein Eingriff erfolgt, desto sicherer wird er.

Die Forderung nach Mindestmengen für die Versorgung von Reifgeborenen in der Geburtshilfe könnte daher der nächste logische Schritt sein. Im Krankenhausreport 2017 fordert die Allgemeine Ortskrankenkasse (AOK) eine Mindestmenge in der Geburtshilfe von 500 Geburten pro Jahr [8]. Für Schleswig-Holstein würde solch eine Mindestmenge eine Schließung von drei weiteren Geburtskliniken in Schleswig, Ratzeburg und Preetz bedeuten (Tabelle 1). 


\begin{tabular}{|c|c|c|c|c|}
\hline & Kreis & $\begin{array}{r}\text { Ver- } \\
\text { sorgungs- } \\
\text { stufe }\end{array}$ & $\begin{array}{r}\text { Geburtenzahl } \\
\text { Einlinge } \\
2016 \\
\end{array}$ & $\begin{array}{r}\text { Fallzahl } \\
\text { gynäkologische Pat. } \\
\text { Vollstationär } 2016\end{array}$ \\
\hline Diako Flensburg & Flensburg & 1 & 943 & 4.797 \\
\hline UKSH Kiel & Kiel & 1 & 1.038 & 4.650 \\
\hline UKSH Campus Lübeck & Lübeck & 1 & 753 & 5.300 \\
\hline WKK Heide & Dithmarschen & 1 & 706 & 3.231 \\
\hline Klinikum Itzehoe & Steinburg & 1 & 886 & 4.650 \\
\hline $\begin{array}{l}\text { Städtisches Krankenhaus } \\
\text { Kiel }\end{array}$ & Kiel & 2 & 1.475 & 5.018 \\
\hline FEK Neumünster & Neumünster & 2 & 1.005 & 2.844 \\
\hline $\begin{array}{l}\text { Imland GmbH - imland } \\
\text { Klinik Rendsburg }\end{array}$ & $\begin{array}{l}\text { Rendsburg- } \\
\text { Eckernförde }\end{array}$ & 2 & 600 & 3.146 \\
\hline $\begin{array}{l}\text { Sana Kliniken Ostholstein } \\
\text { GmbH - Klinik Eutin }\end{array}$ & Ostholstein & 2 & 935 & 3.011 \\
\hline $\begin{array}{l}\text { HELIOS Klinik Schleswig } \\
\text { GmbH }\end{array}$ & $\begin{array}{l}\text { Schleswig- } \\
\text { Flensburg }\end{array}$ & 3 & 361 & 1.574 \\
\hline $\begin{array}{l}\text { Regio Kliniken GmbH - } \\
\text { Klinikum Pinneberg }\end{array}$ & Pinneberg & 3 & 1.077 & 4.223 \\
\hline DRK Ratzeburg & $\begin{array}{l}\text { Herzogtum } \\
\text { Lauenburg }\end{array}$ & 4 & 345 & 855 \\
\hline $\begin{array}{l}\text { Johanniter Krankenhaus } \\
\text { Geesthacht }\end{array}$ & $\begin{array}{l}\text { Herzogtum } \\
\text { Lauenburg }\end{array}$ & 4 & 526 & 2.395 \\
\hline $\begin{array}{l}\text { Klinikum Nordfriesland } \\
\text { Husum }\end{array}$ & Nordfriesland & 4 & 548 & 2.953 \\
\hline $\begin{array}{l}\text { Marienkrankenhaus } \\
\text { Lübeck }\end{array}$ & Lübeck & 4 & 1.101 & 4.318 \\
\hline Klinik Preetz & Plön & 4 & 439 & 1.788 \\
\hline $\begin{array}{l}\text { Imland GmbH - imland } \\
\text { Klinik Eckernförde }\end{array}$ & $\begin{array}{l}\text { Rendsburg- } \\
\text { Eckernförde }\end{array}$ & 4 & 603 & 1.614 \\
\hline $\begin{array}{l}\text { AK Segeberger Kliniken } \\
\text { GmbH }\end{array}$ & Segeberg & 4 & 573 & 2.374 \\
\hline $\begin{array}{l}\text { Paracelsus-Klinik } \\
\text { Henstedt-Ulzburg }\end{array}$ & Segeberg & 4 & 806 & 2.531 \\
\hline $\begin{array}{l}\text { Krankenhaus Reinbek } \\
\text { St. Adolf-Stift GmbH }\end{array}$ & Stormarn & 4 & 723 & 2.936 \\
\hline
\end{tabular}




\section{3) Kosten}

Ein weiterer Grund für die Zentralisierung der Geburtshilfe liegt darin, dass kleinere Geburtskliniken nicht wirtschaftlich arbeiten können, da die Fixkosten für die Aufrechterhaltung der Mindestvoraussetzungen bezüglich Personal und Infrastruktur, wie zum Beispiel die Vorhaltekosten für den Kreißsaal, immer anfallen und eine Klinik erst ab einer bestimmten Fallzahl kostendeckend arbeiten kann.

Augurzky et al. [9] sehen diese kostendeckende Mindestfallzahl für eine gynäkologische Abteilung ab einer Fallzahl von 2.000 pro Jahr erreicht. Diese Fallzahl beinhaltet sowohl geburtshilfliche als auch gynäkologische Fallzahlen. Augurzky et al. [10] konstruieren aufgrund dieser Beobachtung eine Basisklinik, die gerade kostendeckend arbeiten kann. Diese Basisklinik weist eine Patientenstruktur von 485 gynäkologischen und 1.115 geburtshilflichen Fällen auf, davon 432 Geburten. Tabelle 1 ist zu entnehmen, dass Geburtsabteilungen, die weniger als 500 Einlingsgeburten im Jahr haben, auch eine Gesamtfallzahl in der Gynäkologie und Geburtshilfe von weniger als 2.000 pro Jahr aufweisen. Es wird aber auch betont, dass diese Berechnungen nicht für belegärztliche Häuser herangezogen werden können und dass „weiterer Forschungsbedarf in der Regionalisierung der Ergebnisse unter Berücksichtigung der tatsächlich existierenden Klinikstandorte und der regionalen Bevölkerungsdichte (besteht).“ [10]

Zu diesen Kostenproblemen der kleinen Geburtskliniken kommen noch steigende Haftpflichtprämien, sowohl für Hebammen als auch für Ärzte beziehungsweise die Kliniken. Die Berufshaftpflichtversicherung für freiberufliche Hebammen, die Geburtshilfe leisten, stieg zuletzt zum 1. Juli 2018 auf 8.174 Euro pro Jahr. Dieser Betrag ist unabhängig von der Anzahl der begleiteten Geburten. Seit mehreren Jahren verhandeln die Hebammenverbände mit dem GKV-Spitzenverband immer wieder darüber, wie die steigendenden Prämien in die Vergütung der Hebammen einbezogen werden können. Hierbei konnten mehrere Vergütungssteigerungen ausgehandelt werden, 2017 gab es die letzte Anhebung der Vergütung der Hebammenleistungen [11]. Krankenkassen sind darüber hinaus gesetzlich dazu verpflichtet, die steigenden Prämien auszugleichen. Dies geschieht seit dem 01. Juli 2015 gemäß § 134a Abs. 1b SGB V über einen Haftpflichtausgleich, den die Hebammen beantragen können, wenn sie Geburtshilfe leisten und die Qualitätsanforderungen gemäß § 134a Abs. 1 SGB V erfüllen. Die Qualitätsanforderungen beziehen sich auf die Struktur-, Prozess- und Ergebnisqualität. Die konkrete Ausgestaltung dieses Ausgleiches oblag der GKV und den Hebammenverbänden. Uneinigkeit bestand lange bezüglich der Definition der Qualitätskriterien für Hausgeburten. Mit Hilfe einer Schiedsstelle konnte dann zum 25. September 2015 eine Einigung erzielt werden [11]. Durch den Sicherstellungszuschlag soll gewährleistet werden, dass gerade Hebammen in strukturschwachen 
Regionen mit wenigen Geburten finanziell überleben können. Allerdings können diese Bemühungen nicht über die Tatsache hinwegtäuschen, dass der Beruf der freiberuflich arbeitenden Hebamme, die Geburten betreut, finanziell wenig attraktiv ist und eine Hebamme, die wenig Geburten betreut, immer abhängig und auf finanzielle Unterstützung im Rahmen des Sicherstellungszuschlages angewiesen ist. Ein Hinweis darauf könnte sein, dass 2016 weniger als ein Drittel aller freiberuflichen Hebammen in Deutschland auch in der Geburtshilfe tätig waren. Diese Schätzung ist aus der Vertragspartnerliste des GKV Spitzenverbandes zu entnehmen. [12]

Auch in der Ärzteschaft sind steigende Haftpflichtprämien für Geburtshelfer und Geburtshelferinnen zu beobachten. Weiterhin existieren nur noch wenige Anbieter, die überhaupt eine solche Versicherung anbieten. Haftpflichtprämien für Belegärzte überschreiten bei Neuverträgen oftmals 60.000 Euro und auch alte Verträge werden schrittweise diesen Beträgen angepasst [13]. Krankenhäuser übernehmen meist nur einen Teil davon. Dies liegt vor allem daran, dass die Schadenssummen gestiegen sind. Während die Anzahl der Schadensfälle in der Geburtshilfe statistisch gesehen im Vergleich zu anderen Fachrichtungen an letzter Stelle steht, steht die Höhe der Schadensaufwände in der Geburtshilfe an erster Stelle [14]. Genaue Zahlen über geburtshilflich tätige Belegärzte waren trotz eingehender Recherche nicht aufzufinden. Ein Artikel, erschienen im Deutschen Ärzteblatt [15], geht davon aus, dass zwischen 2009 und 2014299 belegärztlich tätige Geburtshelfer ihre Haftpflichtversicherung im Rahmen des Rahmenvertrags zwischen dem Berufsverband der Frauenärzte (BVF) und den Versicherungen R+V Versicherung und Assekuranz AG kündigten [15].

Es wird deutlich, dass kleine Geburtshilfen wirtschaftlich nicht tragfähig sind und auch die Personen, die maßgeblich am Erfolg einer Geburt beteiligt sind, die ärztlichen Geburtshelfer und die Hebammen, sich finanziellen Hindernissen und Herausforderungen stellen müssen.

\subsubsection{Geburtshilfliche Situation auf Föhr vor und nach Schließung der stationären Geburtshilfe}

Ein Zusammenspiel der Faktoren demographischer Wandel, Qualitätssicherung und Kosten der Geburtshilfe, wie im vorherigen Abschnitt beschrieben, hat auch in Föhr mutmaßlich zur Schließung der Geburtshilfestation Anfang Oktober 2015 geführt. Die Schließung der Geburtshilfe von Föhr wurde in der Öffentlichkeit sehr emotional diskutiert und bekam zeitweise auch ein großes mediales Echo. Dieser Abschnitt stellt die wesentlichen Meilensteine im Prozess rund um die Schließung der Geburtshilfe auf Föhr chronologisch dar und vermeidet dabei bewusst eine Bewertung der Handlungen der Akteure im Rahmen der Schließung der Geburtshilfestation.

Vor der Schließung zum 01. Oktober 2015 gab es im Klinikum Nordfriesland, Standort Wyk, einen voll ausgestatteten Kreißsaal sowie eine direkte Anbindung an den Operationssaal für anstehende Sectiones. Es waren zwei freiberufliche Hebammen an das Krankenhaus angebunden. Weiterhin gab 
es zwei belegärztlich tätige Ärzte für Gynäkologie und Geburtshilfe, die die Geburten begleiteten, falls dies nötig war. Eine weitere Hebamme übernahm die Vor- und Nachsorge der Geburten auf Amrum, sie war jedoch nicht als Beleghebamme für Geburtshilfe tätig. Nach der Schließung wurde den betroffenen Frauen empfohlen, sich 14 Tage vor dem errechneten Entbindungstermin auf das Festland zu begeben, vorzugsweise zu den Standorten Flensburg, Heide und Husum, um dort auf die Geburt zu „warten“.

Die Schließung der Geburtshilfe auf Föhr gestaltete sich als ein komplexer Prozess, der auf Landesebene in Schleswig-Holstein schon im Jahr 2014 begann. Mit dem Bericht der Landesregierung "Situation und Perspektiven der Geburtshilfe in Schleswig-Holstein" [16] verfasste die Landesregierung einen Status Quo der Geburtshilfe in Schleswig- Holstein und zeigte nötigen Handlungsbedarf auf. Schon in diesem Bericht wurde klar, dass vor allem der Kreis Nordfriesland aufgrund der geographischen und demographischen Lage besonders berücksichtigt werden sollte. Die Kreise Nordfriesland und Ostholstein wurden als Modellregionen benannt und hier sollten Konzepte greifen, die die regionalen Versorgungsstrukturen stärken sollten. Diese beinhalteten zum Beispiel Schulungsmaßnahmen bei geburtshilflichen Notfällen im Rettungsdienst, den Ausbau und die Überprüfung von Boarding Einrichtungen sowie eine Verbesserung der Netzwerkstrukturen zwischen Ärzten, Hebammen und Geburtskliniken. Für die Umsetzung dieser Konzepte wurde vom Landtag eine Summe von 45.000 Euro bereitgestellt.

Im Herbst 2014 ließ das Klinikum Nordfriesland gGmbH die Geburtshilfestation in Wyk auf Föhr von zwei Gutachtern in Hinblick auf die Mindestanforderungen an Prozess-, Struktur- und Ergebnisqualität in der Geburtshilfe prüfen und einen Bericht anfertigen. Dieser Bericht lag der Öffentlichkeit bis zur Schließung des Kreißsaals und auch noch darüber hinaus nicht vor. Erst im Jahr 2016 wurde eine Teilabschrift veröffentlicht [17]. In dem Abschnitt, der öffentlich ist, kommen die Gutachter zu dem Schluss, dass „die Klinik nur ansatzweise den geforderten Mindeststandards für den Betrieb einer geburtshilflichen Abteilung einer Klinik der Grund- und Regelversorgung stand[hält].“ Aufgrund dessen sei das Haftungsrisiko als „ausgesprochen hoch einzustufen.“

Im August 2015 veröffentlichte die Landesregierung im Auftrag des Landtags einen Bericht zur „Versorgung von Schwangeren und Neugeborenen in Schleswig-Holstein“ [18], in dem die Situation der Geburtshilfe in Schleswig- Holstein in aktualisierter Form aufgezeigt wird und die Erfüllung von Qualitätskriterien, Perspektiven und Handlungsmöglichkeiten diskutiert wird. Zusätzlich werden Expertenmeinungen von Akteuren der Geburtshilfe integriert, die kontroverse Positionen beziehen und abermals verdeutlichen, wie sensibel das Thema ist. Diese werden im Folgenden erläutert. 
Zunächst gibt es die Expertenmeinungen, darunter unter anderem eine Stellungnahme der DGGG und eine Stellungnahme der Klinik für Frauenheilkunde des Universitätsklinikums Kiel (UKSH), die deutlich machen, dass die Abweichung von Mindeststandards auch in der Versorgungsstufe 4 als Risiko für Kind und Mutter zu bewerten ist. Die DGGG schreibt dazu: „Abweichungen von den Mindeststandards bedeuten eine potenzielle Gefährdung der Gesundheit und des Lebens von Mutter und Kind. Dies kann keine akzeptable Lösung der regionalen Versorgungsprobleme darstellen." [18] Die Beurteilung der Situation seitens der Klinik für Frauenheilkunde am UKSH Kiel lautet: „Die Aufweichung von Mindeststandards bedeutet, Zugeständnisse hinsichtlich einer Verschlechterung der Versorgungsqualität einzugehen." [18]. Prof. Dr. Rolf Kreienberg, Gynäkologe und seit Mai 2015 Präsident der Arbeitsgemeinschaft der Wissenschaftlichen Medizinischen Fachgesellschaften (AWMF), weist aber auch darauf hin, dass „die Erfüllung der Mindestanforderungen entsprechend der Leitlinie der DGGG in erster Linie ein finanzielles Problem [ist]. “ [18]

Dagegen hält Dr. Achim Niesel, damaliger Chefarzt der Geburtshilfe und Gynäkologie in der Klinik Preetz, vor, dass „aufgrund der aktuellen Daten der Perinatalstatistik Schleswig-Holstein [nicht] von einer schlechteren medizinischen Qualität in den kleineren Geburtshilfen auszugehen [ist]." [18] Die Deutsche Gesellschaft für Hebammenwissenschaft e.V. (DGHWi) zitiert Studien, die für eine „Effektivität lokaler und kontinuierlicher Betreuungsstrukturen und die Qualität von hebammengeleiteter Versorgung [sprechen].“ [18]

Des Weiteren wird in dem Bericht zur "Versorgung von Schwangeren und Neugeborenen in SchleswigHolstein“ die Entwicklung und Umsetzung eines Maßnahmenkatalogs in Auftrag gegeben, der von der OptiMedis AG bis zum 1. Quartal 2016 durchgeführt werden sollte. Auftraggeber ist das Ministerium für Soziales, Gesundheit, Wissenschaft und Gleichstellung in Schleswig-Holstein. Das erstellte Gutachten, das im Mai 2016 veröffentlicht wurde, beleuchtet und bewertet die geburtshilfliche Situation in Schleswig-Holstein und schlägt der Landesregierung verschiedene Maßnahmen zur Verbesserung der Geburtshilfe in Schleswig-Holstein vor. Diese Maßnahmen werden im Diskussionsteil der vorliegenden Arbeit aufgegriffen.

In einer Pressemitteilung des Klinikum Nordfriesland wird am 15. September 2015 bekannt gegeben, dass die Geburtshilfe auf der Insel Föhr Ende November 2015 schließt [19]. Der Aufsichtsrat folgt damit einstimmig der Entscheidung des Geschäftsführers, die geburtshilfliche Versorgung in der Klinik Wyk auf Föhr einzustellen. Die offizielle Begründung für die Schließung fußt maßgeblich auf den Ergebnissen des Gutachtens bezüglich der nicht als ausreichend empfundenen Einhaltung der Mindeststandards und das dadurch erhöhte Haftungsrisiko. Finanzielle Gründe werden auch auf spätere Nachfragen von verschiedenen Kreistagsfraktionen verneint [20]. Allerdings beziffert Dieter 
Harrsen, Landrat und Vorsitzender des Aufsichtsrates des Klinikums Nordfriesland, in einem Schreiben an die Mitglieder des Kreistages und die Mitglieder des Arbeits- und Sozialausschusses, dass eine Weiterführung einer Inselgeburtshilfe, die alle Mindestanforderungen erfüllt, circa 1.709.000 Euro Mehrkosten verursachen würde [21].

Am 02. Oktober 2015 wurde in einer Pressemittelung des Klinikum Nordfriesland bekannt gegeben, dass die Geburtshilfe auf der Insel Föhr bereits rückwirkend zum 01. Oktober 2015 schließt [22]. Dies sei dadurch bedingt, dass die gynäkologischen Belegärzte sich aufgrund des erhöhten Haftungsrisikos dazu entschlossen hätten, keine Geburten mehr durchzuführen. Eine der Belegärztinnen, Dr. Juliane Engel, schrieb dazu: „Unsere Entscheidung, die Geburtshilfe auf Föhr zunächst einzustellen, fußte auf dem Rat der von uns konsultierten RA-Kanzlei, dass durch die Aussage der Klinikleitung, organisationsrelevante Strukturen aktuell nicht vorzuhalten, bei einem möglichen Schadensfall aus dem dann resultierenden Übernahmeverschulden für uns erhebliche Konsequenzen hätten erwachsen können." [23]

Die Schließung löste eine Welle von Unmut in der Bevölkerung und auch in der politischen Opposition aus. Zahlreiche offene Briefe wurden geschrieben, Bürgerinitiativen ins Leben gerufen, politisch diskutiert und medial, auch auf nationaler Ebene [24-26], kommentiert. Mehrere Versuche, die Geburtshilfe zeitnah wieder zu eröffnen, scheiterten.

Abbildung 3: Chronologie der Schließung der Geburtshilfe auf Föhr

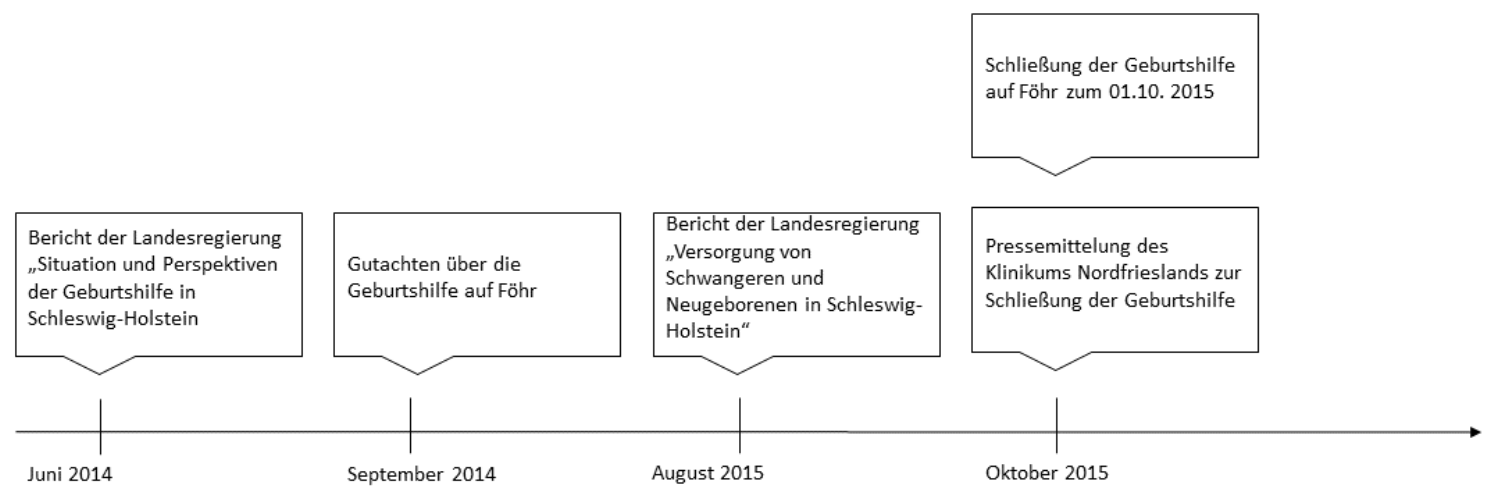




\subsection{Aktueller Forschungsstand}

Die möglichen Gründe für die Verlagerung der Geburtshilfe in größere spezialisierte Zentren wurden in den vorherigen Kapiteln diskutiert. Auch die Literatur beschäftigt sich mit diesem Wandel. Im Rahmen der Literaturrecherche fanden sich dabei drei zentrale Forschungsgebiete: der medizinische, der psychosoziale und der gesundheitsökomische Aspekt der Zentralisierung der geburtshilflichen Versorgung. Diese drei Forschungsgebiete sollen im Folgenden näher beleuchtet werden.

Einigkeit herrscht in zahlreichen wissenschaftlichen Studien darin, dass die perinatale Mortalität und Morbidität bei der Untergruppe „Hochrisikogeburten“ durch die Versorgung in spezialisierten und somit zentralisierten Geburtskliniken sinken. Dies wird in einer Metaanalyse von Lasswell [27] deutlich. Hochrisikogeburten werden in der Arbeit von Lasswell [27] definiert als Lebendgeborene mit einem Geburtsgewicht von unter 1.500g oder einem Gestationsalter von unter 32 Wochen.

Uneinigkeit herrscht allerdings darüber, ob die perinatale Mortalität und Morbidität bei Normalgeburten ebenso mit einer Versorgung in großen Perinatalzentren sinken würde und somit Schließungen aus medizinischer Sicht indiziert sind. [28-30]

Eine Studie wird in diesem Zusammenhang immer wieder zitiert. Es handelt sich dabei um die Studie von Heller (2002). Die Daten hierfür stammen aus der hessischen Perinatalstatistik zwischen 1990 und 2000. Heller kommt zu dem Resultat, dass sich das Mortalitätsrisiko in Krankenhäusern mit weniger als 500 Geburten pro Jahr im Vergleich zu Krankenhäusern mit mehr als 1.500 Geburten pro Jahr verdreifacht. Heller sah damals schon die Notwendigkeit der Umstrukturierung der Geburtshilfe in Deutschland, um das Risiko der frühen neonatalen Mortalität zu senken.

Zu einem ähnlichen Ergebnis kam eine schwedische Studie [28]. Die Mortalität von NichtRisikogeburten innerhalb der ersten 28 Tage nach Geburt sank, je höher die Zentralisierung und Zugang zur neonatalen Versorgung war. Merlo sieht somit eine Zentralisierung der Geburtshilfe aus strikt medizinischer Sicht für vertretbar. Dennoch weist der Autor darauf hin, dass es unabdingbar ist, mögliche Schließungen aus einer ganzheitlichen und gesundheitsökonomischen Perspektive zu analysieren. [28]

Andere Studien hingegen sehen keine erhöhte Mortalität bei Nicht-Risikogeburten in kleineren Geburtshilfen [31, 29]. Grytten [31] argumentiert, dass kleine Geburtshilfen eine Berechtigung haben, Normalgeburten durchzuführen, solange sie durch qualitativ hochwertige Betreuung in der Lage sind, Hochrisikogeburten zu filtern und von vornerein an entsprechende spezialisierte Zentren weiterzuleiten. Der Forscher weist auch darauf hin, dass die meisten kleinen geburtshilflichen Einrichtungen in Norwegen zwischen 1980 und 1990 aus ökonomischen Gründen und nicht aufgrund 
von Qualitätsmängeln geschlossen wurden. Es sei daher auch weiterhin nötig, kontinuierlich die Wirtschaftlichkeit der kleineren Geburtshilfeabteilungen zu bewerten.

Tracy et al. [29] analysieren ebenfalls, ob die Zahl der durchgeführten Geburten eines Krankenhauses in Australien Einfluss auf die perinatale Mortalität von Normalgeburten hat. Auch in dieser Studie konnte kein inverser Effekt von Krankenhausgröße auf die perinatale Mortalität nachgewiesen werden. Darüber hinaus finden in den von Tracy beobachteten Krankenhäusern mit einer Geburtenzahl von weniger als 100 Geburten pro Jahr weniger Geburtseinleitungen, weniger intrathekale Anästhesien, weniger sekundäre Sectiones und weniger instrumentelle Geburten statt.

Ein zweites wissenschaftliches Forschungsfeld in diesem Kontext beleuchtet die psychosozialen Konsequenzen für die Mütter, wenn keine wohnortnahe Geburtshilfe stattfindet.

Kornelsen et al. [32] resümieren, dass das Aufgeben einer wohnortnahen Geburtshilfe erhebliche psychische Folgen für die betroffenen Frauen hat. In einer Folgestudie [33] werden diese psychischen Folgen konkretisiert: Gebärende Frauen, die mehr als eine Stunde zum Ort der Geburt fahren müssen, haben ein erhöhtes Stress- und Angstlevel verglichen mit Frauen, die wohnortnah versorgt werden können. Kornelsen et al. [33] machen ebenfalls darauf aufmerksam, dass den Frauen durch den Aufenthalt außerhalb ihres Wohnortes finanzielle Nachteile entstehen und vor allem sozial schwache Familien benachteiligt sind.

Besonders deutlich wird die Situation in einer Studie von Roach [34], die die Situation der Gebärenden in den australischen Territorien im Indischen Ozean aufarbeitet. Seit 1998 müssen auch dort die Frauen die Inseln in der 36. Schwangerschaftswoche verlassen und zur Geburt auf das Festland fliegen. In der qualitativen Analyse von Roach [34] wird deutlich, dass die betroffenen Frauen erhebliche soziokulturelle und finanzielle Bürden auf sich nehmen müssen, um zur Geburt auf das Festland zu kommen. Die finanziellen Schwierigkeiten entstanden hauptsächlich durch die Kosten des "Umzugs" auf das Festland für mehrere Wochen und den Verdienstausfall während dieser Zeit.

Ebenso wird die Notwendigkeit in der aktuellen Literatur erkannt, gesundheitsökonomische Aspekte in der gynäkologischen und geburtshilflichen Versorgung zu diskutieren [35, 36]. Hierzu wird auf die Ausführungen im vorherigen Kapitel verwiesen.

Aus der vorangegangen Literaturrecherche wird deutlich, dass die Diskussionen rund um den möglichen Zusammenhang zwischen perinataler Mortalität bei Nicht-Risikogeburten und der Krankenhausgröße noch nicht abgeschlossen sind. Eine Schließung kleinerer Geburtshilfeabteilungen aus medizinischer Sicht bleibt daher noch diskussionswürdig. Einigkeit hingegen herrscht in dem Punkt, dass gerade kleine Geburtshilfeabteilungen zunehmend höheren Kostendrücken ausgesetzt sind und 
eine Evaluierung der bisherigen Strukturen erfolgen muss. Ein weiterer Aspekt, der in der Forschung Beachtung findet, sind die psychosozialen Folgen der werdenden Eltern, wenn keine wohnortnahe Geburtshilfe vorhanden ist.

Auffällig ist jedoch auch, dass in keiner der analysierten Studien eine Quantifizierung der Kosten für die betroffenen Eltern, wenn vor Ort keine Geburtshilfe mehr stattfinden kann, erfolgt. Viele Studien sprechen zwar finanzielle Schäden für die Familien im Rahmen der Schließung der wohnortnahen Geburtshilfe an, jedoch findet keine Quantifizierung der Kosten statt [33, 34]. Merlo et al. [28] fordert explizit eine genaue Aufarbeitung der Kosten, die den Patientinnen nach Schließung der Geburtshilfe entstehen.

Um diesen Forderungen zu entsprechen, bedarf es einer konkreten wissenschaftlichen und aktuellen Aufarbeitung der Faktoren, die Frauen nach der Schließung einer wohnortnahen Geburtshilfestation bewältigen müssen und vor allem einer Quantifizierung der Kosten, die auf die Frauen zukommen. Nur so ist es möglich, eine umfassende gesundheitsökomische Evaluation zu erhalten.

Keine der oben genannten Studien konnte bisher die Kosten, die den Frauen nach der Schließung der Geburtshilfestation im ländlichen Raum entstehen, quantifizieren. Eine wesentliche Aufgabe der vorliegenden Arbeit ist es, diese „Lücke“ in der bisherigen Forschung zu schließen.

\subsection{Fragestellung und Zielsetzung der vorliegenden Arbeit}

Ziel der vorliegenden Arbeit ist es, die Schließung der Geburtshilfestation auf Föhr in einen gesundheitsökonomischen Zusammenhang zu stellen. Dafür wird eine Aufarbeitung der Schließung der Geburtshilfestation auf Föhr in drei Schritten erfolgen.

(1) Es werden die anfallenden direkten und indirekten Kosten der Insulanerinnen quantifiziert. Zudem werden auch die direkten medizinischen und direkten nicht-medizinischen Kosten der weiteren Kostenträger erfasst, die im Zusammenhang mit der Schließung der Geburtshilfe auftreten.

(2) Durch (1) ist es möglich, einen Vorher-Nachher-Vergleich zu erstellen und mögliche inkrementelle Kosten oder Kostenverschiebungen zu erkennen.

(3) Darüber hinaus soll geprüft werden, ob die Kostenerfassung dieser regionalen Analyse gesundheitsökonomische Perspektiven für ähnliche Fälle in der Zukunft aufzeigen kann. 


\section{Material und Methoden}

\subsection{Studiendesign und - planung}

Die vorliegende Dissertation „Direkte und indirekte Kosten einer Geburt bei Insulanerinnen, wenn vor Ort keine Geburtshilfe möglich ist" ist ein Teil eines größeren Projektes zum Thema "Geburtserfahrungen von Insulanerinnen nach Schließung der stationären Geburtshilfe auf Föhr", in welches eine weitere Promotionsstudentin einbezogen war. Das Studiendesign umfasst einen qualitativ-empirischen Teil sowie einen quantitativ-empirischen Teil. Diese Methodenkombination kann auch als sequenzielles quantitativ-qualitatives Design beschrieben werden [37]. Zur primären Datensammlung wurde das Studiendesign zusammen mit der zweiten Promotionsstudentin erarbeitet und der erste Teil, die semi-strukturierten Interviews, wurden gemeinsam durchgeführt.

Der qualitativ-empirische Teil, in dem semi-strukturierte Interviews geführt wurden, war nötig, um einen Überblick über das gesellschaftlich und emotional hoch komplexe Thema zu bekommen und zu filtern, welche Hypothesen und Theorien bestehen und gegebenenfalls vertieft analysiert werden sollten [38]. Darauf aufbauend wurde ein quantitativ-empirischer Teil in Form einer Online-Umfrage entwickelt, um die erarbeiteten Kategorien und Hypothesen aus den Interviews auf eine mögliche „Verallgemeinerbarkeit und Geltungsreichweite“ [37] hin zu überprüfen.

Parallel dazu wurden relevante Kostenkomponenten vor, aber vor allem nach der Schließung der Geburtshilfestation auf Föhr identifiziert, so dass die Arbeit mit diesen drei erarbeiteten Teilen in einen gesundheitsökonomischen Kontext gestellt werden kann.

\subsection{Gesundheitsökonomische Definitionen}

Gesundheitsökonomie ist eine interdisziplinäre Wissenschaft, die sich mit der Knappheit von Gesundheitsleistungen auseinandersetzt [39]. Dabei befindet sie sich permanent in einem Spannungsfeld von Politik, Wirtschaft und Recht. Vor diesem Hintergrund und auch der Tastsache, dass medizinische Leistungen nicht unbegrenzt verfügbar sind, sind gesundheitsökonomische Evaluationen von hoher Relevanz. Gesundheitsökomische Evaluationen versuchen, medizinische Maßnahmen nach ökonomischen Maßstäben zu bewerten [40] und eine möglichst effiziente Verteilung von gesundheitlichen Ressourcen zu erreichen. Die Evaluationen machen es auch möglich, gesundheitspolitische Empfehlungen auszusprechen und somit wieder eine Brücke zwischen Politik, Wirtschaft und Recht zu schlagen.

Wie in 1.3 formuliert, ist es Ziel dieser Arbeit, die Schließung der stationären Geburtshilfe auf Föhr in einen gesundheitsökonomischen Kontext zu stellen und die potenziellen Mehrkosten, die nach der Schließung entstanden sind unter einer gesellschaftlichen Perspektive zu evaluieren. Dafür ist es nötig, 
eine für diese Arbeit relevante Taxonomie der Kosten aufzustellen. Zur Ermittlung der Kosten wurde wie folgt vorgegangen [41, 40]: Zuerst wurden die relevanten Kostenkomponenten identifiziert, danach die Ressourcenverbräuche gemessen und abschließend erfolgte eine monetäre Bewertung der Ressourceneinheiten.

\subsubsection{Identifikation relevanter Kostenkomponenten}

Die relevanten Kostenkomponenten wurden zunächst mittels der Durchführung der qualitativen Interviews ermittelt. Mit Hilfe der qualitativen Inhaltsanalyse konnten erste Kostenkomponenten der Studienteilnehmerinnen, aber auch der weiteren beteiligten Akteure erfasst werden. Parallel wurde auch in der Literatur nach Studien mit ähnlichen Rahmenbedingungen [33] gesucht und gefiltert, welche Kosten für unsere Studie zu berücksichtigen sind. Die erarbeiteten Kostenkomponenten sind in Abbildung 1 dargestellt, die in Anlehnung an Fleßa [39] erstellt wurde. 


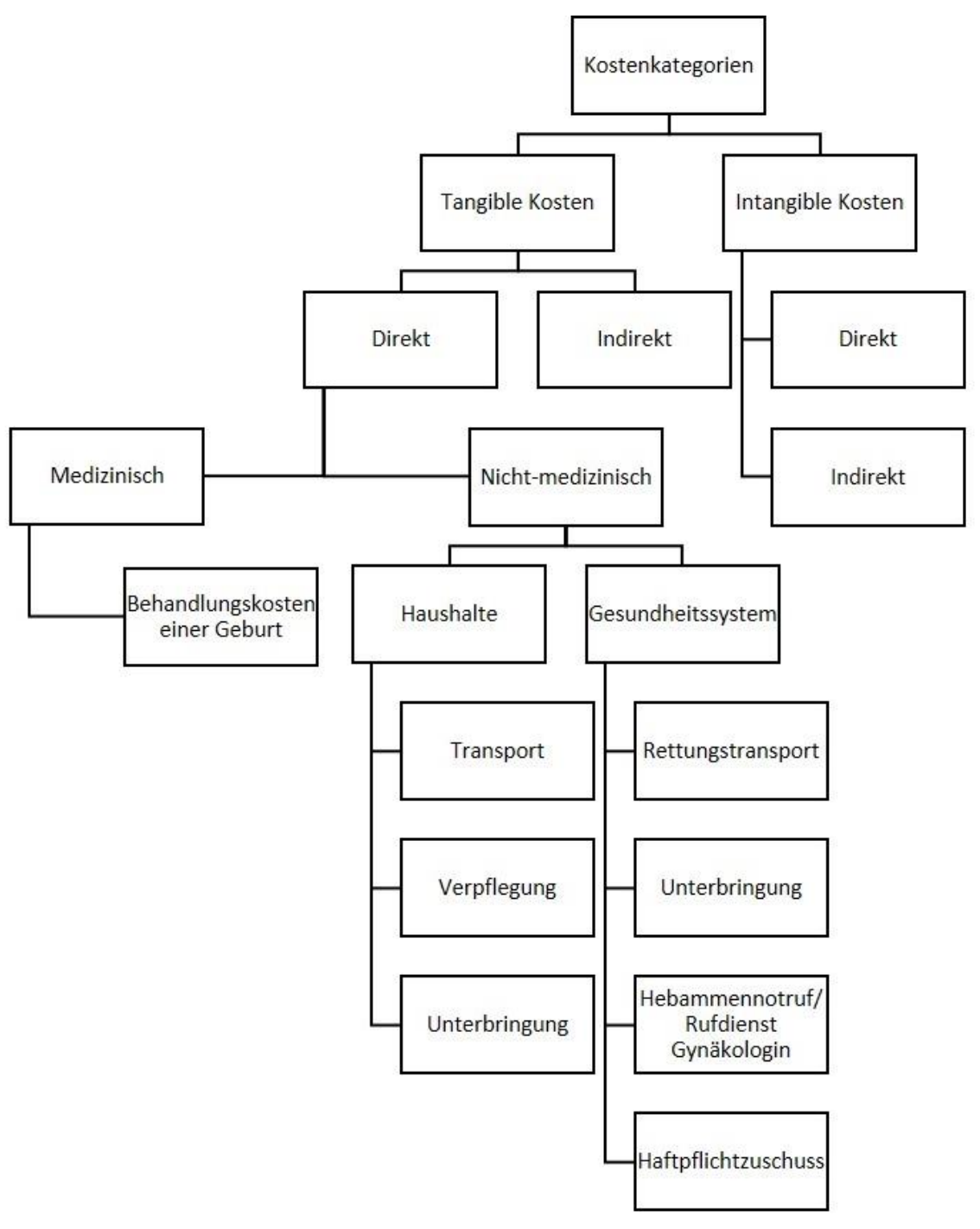

In der Gesundheitsökonomie kann man zunächst unter tangiblen und intangiblen Kosten differenzieren. Die intangiblen Kosten beinhalten zum Beispiel Schmerzen (direkte intangible Kosten) oder Einbußen der Lebensqualität (indirekte intangible Kosten) nach einer erfolgten medizinischen Intervention [42]. Diese Kosten sind nur sehr schwer in monetäre Einheiten zu fassen, werden aber auch durch gesundheitsökonomische Evaluation erfasst. Für die vorliegende Dissertation jedoch wurden diese Kosten nicht berücksichtigt, da diese Dissertation den Hauptfokus darauf legt, monetäre Kosten zu identifizieren und zu bewerten.

Die tangiblen Kosten beinhalten Kosten, die in der Regel problemlos monetär bewertet werden können. Diese Kosten können entweder direkt oder indirekt entstehen. Die Differenzierung zwischen indirekten und direkten Kosten ist eine weitere Möglichkeit, Kosten in gesundheitsökonomischen Evaluationen zu kategorisieren. 


\subsubsection{Direkte medizinische und direkte nicht-medizinische Kosten}

Unter den direkten Kosten versteht man den direkt zuordnungsbaren Ressourcenverzehr, den man in Geldeinheiten bewerten kann [40]. In der Gesundheitsökonomie werden unter den direkten Kosten die direkten nicht-medizinischen Kosten und die direkten medizinischen Kosten zusammengefasst [40]. Die direkten medizinischen Kosten stehen in unmittelbarem Zusammenhang mit der Durchführung der medizinischen Leistung, wie zum Beispiel einem Krankenhausaufenthalt. Die direkten nicht-medizinischen Kosten entstehen aus den Folgen medizinischer Interventionen. Hierbei handelt es sich etwa um Rettungstransportkosten.

In der vorgelegten Dissertation werden als direkte medizinische Kosten die Kosten einer Geburt im „Diagnosis Related Groups“ (DRG) System, im einheitlichen Bewertungsmaßstab als Vergütungssystem der vertragsärztlichen Versorgung (EBM) und im Hebammenhilfevertrag erfasst. Näheres dazu wird in Kapitel 2.4. erläutert.

Direkte nicht-medizinische Kosten dieser Studie werden noch einmal differenziert in die direkten nichtmedizinischen Kosten der Haushalte, also der privaten Haushalte der werdenden Eltern, sowie in die direkten nicht-medizinischen Kosten des Gesundheitssystems. Es handelt sich bei den Kosten der privaten Haushalte ausschließlich um selbst getragene Kosten der betroffenen Familien, die unmittelbar im Zusammenhang mit dem Festlandaufenthalt zur Geburt des Kindes entstanden sind. Bei den direkten nicht-medizinischen Kosten handelt es sich um die Kosten, die den Kostenträgern des Gesundheitssystems unmittelbar im Zusammenhang mit dem Festlandaufenthalt einer Föhrer Frau zur Geburt ihres Kindes entstanden sind.

Unter die direkten nicht-medizinischen Kosten der privaten Haushalte fallen hauptsächlich Transport-, Verpflegungs- und Unterbringungskosten. Die Transportkosten enthalten alle Kosten, die angefallen sind, um zur Entbindung auf das Festland zu kommen. Sie enthalten daher Kosten für die Fährfahrt sowie Park- und Benzinkosten.

Als Verpflegungskosten werden nur die Verpflegungskosten aufgenommen, die zusätzlich zu den von den Krankenkassen übernommenen Verpflegungskosten entstanden sind.

Als Unterbringungskosten werden die Kosten ermittelt, die für die Unterkunft auf dem Festland entstanden sind. Darunter fallen zum Beispiel die Kosten für den Partner für das Boarding, wenn dieser nach der Geburt des Kindes weitere Nächte in dem Boarding Haus verbracht hat. Aus den Interviews geht hervor, dass die Krankenversicherung zwar den Aufenthalt im Boarding Haus für die Mütter und Partner zahlt, jedoch nur bis zur Geburt des Kindes. Weitere, über die Geburt hinaus- gehende Aufenthaltskosten des Partners im Boarding Haus werden nicht übernommen. Als 
Unterbringungskosten werden auch Kostenzuschüsse zur Unterkunft der werdenden Mütter gerechnet, wenn diese bei Freunden oder der Familie auf dem Festland untergekommen sind.

Unter die direkten nicht-medizinischen Kosten des Gesundheitssystems fallen die Kosten für den Rettungstransport, die Kosten für die Unterbringung der Mütter in den Boarding Einrichtungen der jeweiligen Entbindungskliniken sowie die Kosten des Hebammennotrufs und des geburtshilflichen Bereitschaftsdienstes auf der Insel Föhr. Ebenfalls fallen unter diese Kategorie Zuschüsse zu den Haftpflichtversicherungen der Hebammen und der Gynäkologin. Der Rettungstransport kann auf drei verschiedenen Wegen erfolgen. Per Rettungstransport auf der Fähre, per Seenotrettungskreuzer oder per Luftrettung durch einen Helikopter.

\subsubsection{Indirekte Kosten}

Die indirekten Kosten sind Kosten, die durch „volkswirtschaftlichen Produktionsverlust zum Beispiel durch krankheitsbedingte Abwesenheit vom Arbeitsplatz, Invalidität oder vorzeitigen Tod" [40] entstehen. Indirekte Kosten entstanden bei der vorliegenden Dissertation immer dann, wenn es bei Selbstständigkeit durch die, im Vergleich zu der Situation vor der Schließung der stationären Geburtshilfe, frühzeitige Reise auf das Festland zu Einkommensverlusten kam, entweder der werdenden Mutter oder des Partners oder beider. Hierbei ist auch die spezielle Arbeitsmarktsituation auf der Insel Föhr zu berücksichtigen, da viele Föhrer/innen selbständig in kleinen Handwerksbetrieben oder in der Gastronomie arbeiten. Ein mehrtägiges Schließen des Betriebes bedeutet für sie einen finanziellen Aufwand. Indirekte Kosten wurden auch berücksichtigt, wenn der Partner extra für die Begleitung der Partnerin auf das Festland „krank machen“ musste. Auch hier handelt es sich um eine Verringerung der gesamtwirtschaftlichen Produktivität, die es vor der Schließung der stationären Geburtshilfe auf Föhr nicht gegeben hätte, da der Partner vor Ort gewesen wäre.

\subsubsection{Das DRG-System}

Die diagnosebezogenen Fallgruppen (DRGs) sind die Basis des „durchgängigen, leistungsorientierten und pauschalisierenden Vergütungssystems“ ( $\$ 17 b$ KHG), nach dem die deutschen Akutkrankenhäuser seit 2004 verpflichtet sind, stationäre Leistungen abzurechnen. Optimalerweise sollte das Entgelt den tatsächlich entstandenen Kosten entsprechen. Die DRGs folgen dabei einem Fallklassifikationssystem, in dem klinische Patientengruppen gebündelt werden, die in ihren Behandlungskosten, also ökonomisch, und medizinisch-klinisch als homogen anzusehen sind [43]. Die Gruppierungsmerkmale folgen dabei den Diagnosen nach ICD-10 (Internationale statistische Klassifikation der Krankheiten und verwandter Gesundheitsprobleme) sowie Operationen und Prozeduren nach dem Operations- und Prozedurenschlüssel-301 (OPS) (§§ 295, 301 Sozialgesetzbuch V). Die deutschen Kodierrichtlinien, herausgegeben von der Deutschen Krankenhausgesellschaft (DKG), dem Spitzenverband der 
gesetzlichen Krankenversicherungen (GKV), dem Verband der privaten Krankenversicherung (PKV) und dem Institut für das Entgeltsystem im Krankenhaus (InEK GmbH) ergeben sich aus den OPS-301 und den ICD-10 Kodierungen. Die DRGs werden nach den deutschen Kodierrichtlinien verschlüsselt.

Eine DRG besteht aus einer Buchstaben-Zahlen-Kombination mit insgesamt vier Ziffern. Die erste Ziffer repräsentiert die Hauptdiagnose, die sogenannte Major Diagnostic Category (MDC). Die MDCs sind im Wesentlichen nach dem Organsystem definiert, auf das sich die Hauptdiagnose stützt und können Werte zwischen A und Z annehmen. Die Hauptdiagnose "O" repräsentiert die Kategorie „Schwangerschaft, Geburtshilfe, Wochenbett". Die folgenden zweiten und dritten Stellen einer DRG unterteilt die MDC in eine operative (01-39), andere (40-59) oder medizinische (60-99) Partition, die sogenannten sub-MDCs. Aus diesen drei Ziffern resultiert die Basis-DRG. Die vierte Stelle einer DRG kennzeichnet den Schweregrad innerhalb der Basis-DRG. Dabei bezeichnet „ $A$ “ den höchsten Schweregrad und damit auch den höchsten Ressourcenverbrauch, "B“ den zweithöchsten etc. „"“ kennzeichnet den niedrigsten Schweregrad. Die Zuteilung zum Schweregrad erfolgt unter anderem durch den „Patient Clinical Complexity Level“ (PCCL). Alle relevanten Nebendiagnosen tragen zur Ermittlung des Gesamtschweregrads einer Begleiterkrankung (CCL) bei. Durch die Verrechnung aller CCLs ergibt sich dann schließlich die PCCL, der medizinische, fallbezogene Schweregrad. Dieser wiederum kann Werte zwischen 0 (Fall ohne Komplikation) und 4 (äußerst schwere Komplikation und Begleiterkrankung) annehmen. Diese Zuordnungen werden anhand einer speziellen "Grouper"Software in der Klinik übernommen. Jedem stationären Patienten wird nach seiner Entlassung genau eine DRG zugeordnet. Diese berücksichtigt neben aller abgelaufenen Diagnostik und Therapie, die nach den Kodierleitlinien verschlüsselt werden dürfen, des Weiteren auch persönliche Daten, Aufnahmeund Entlassart, Beatmungszeiten, Verlegungen und die Dauer des stationären Aufenthalts.

Der Aufbau einer DRG wird anhand einer relevanten DRG dieser Dissertation im Folgenden kurz dargestellt.

Tabelle 2: Aufbau einer DRG als Beispiel im Rahmen der Geburtshilfe

\begin{tabular}{|l|l|}
\hline O60D & $\begin{array}{l}\text { Vaginale Entbindung ohne komplizierende Diagnose, Schwangerschaftsdauer mehr als } \\
33 \text { vollendete Wochen }\end{array}$ \\
\hline O & MDC 14: Schwangerschaft, Geburt und Wochenbett \\
\hline 60 & Sub-MDC: medizinische Partition (60-99) \\
\hline D & Schweregrad \\
\hline
\end{tabular}


Weitere DRGs, mit denen im Rahmen der vorliegenden Dissertation gearbeitet wird, sind:

- O01E: Sekundäre Sectio caesarea mit komplizierender Diagnose, Schwangerschaftsdauer mehr als 33 vollendete Wochen (SSW), ohne komplexe Diagnose

- O01H: Primäre Sectio caesarea ohne komplizierende Diagnose, Schwangerschaftsdauer mehr als 33 vollendete Wochen (SSW), ohne komplexe Diagnose

Das DRG-System wird nicht nur zur Klassifikation von Diagnosen benutzt, sondern auch als Abrechnungseinheit der sogenannten Fallpauschale. Jeder Basis-DRG ist eine bestimmte Bewertungsrelation, das Relativ- oder Kostengewicht, zugeordnet, die jährlich auf Basis der Kostenkalkulationen von Stichprobenkrankenhäusern des Instituts für Entgeltsystem im Krankenhaus (InEK) ermittelt werden. Die Kostenzurechnung folgt dabei einem Vollkostenansatz auf Ist-KostenBasis. Zusatzentgelte werden nicht berücksichtigt. Je höher der durchschnittliche Behandlungsaufwand ist, desto höher ist die Bewertungsrelation.

Um das Entgelt pro DRG zu bestimmen, wird das relative DRG-spezifische Kostengewicht mit einem landesspezifischen Basisfallwert (Tabelle 3) multipliziert. Ab dem Jahr 2009 gilt in jedem Bundesland ein einheitlicher Basisfallwert, der jährlich neu von den Landesverbänden der Krankenkassen und den Landeskrankenhausgesellschaften ausgehandelt wird. Die Berechnung des Basisfallwertes übernimmt die InEK. Das Entgelt im DRG System wird nach folgender Gleichung bestimmt:

Landesbasisfallwert x Bewertungsrelation = Entgelt im DRG System

Zudem erhalten Krankenhäuser nur die volle Pauschale, wenn der Fall innerhalb der unteren und oberen Grenzverweildauer der betreffenden DRG liegt. Die Grenzverweildauern werden durch die Kalkulationsdaten der InEk ermittelt. 


\subsection{Kosten einer Geburt}

In der vorliegenden Dissertation wurden die direkten medizinischen Kosten einer Geburt zunächst über das G-DRG System ermittelt. Dabei wurden jeweils für die DRGs O60D, O01E und O01H die Bewertungsrelationen der Jahre 2010 bis 2015 aus den zugehörigen Fallpauschalkatalogen mit den Landesbasisfallwerten ohne Ausgleiche von Schleswig-Holstein der Jahre 2010 bis 2015 multipliziert, um ein durchschnittliches DRG-spezifisches Entgelt zu erhalten (Tabellen 3 und 4). Dieses Entgelt spiegelt nicht den Ressourcenverbrauch und die tatsächliche Kostenstruktur des Krankenhauses wider, sondern die direkten medizinischen Kosten einer Geburt aus Kostenträger- bzw. Krankenkassenperspektive.

Weiterhin wurde bei den Abrechnungsdaten zwischen Hauptabteilungen, Hauptabteilungen mit Beleghebammen sowie Belegabteilungen mit Belegoperateur und Beleghebamme unterschieden. Die stationäre Geburtshilfe auf Föhr war bis zur Schließung Ende September 2015 eine belegärztlich geführte Geburtshilfe. Das DIAKO Flensburg verfügt über eine geburtshilfliche Hauptabteilung mit angestellten Hebammen, das WKK Heide verfügt sowohl über Beleg- als auch angestellte Hebammen [16]. Maßgeblicher Unterschied zwischen Beleg- und Hauptabteilung in der Berechnung der direkten medizinischen Kosten sind die Personalkosten, da belegärztliche Tätigkeiten und die Kosten der Versorgung durch eine Hebamme laut des Kalkulationshandbuchs 4.0 der InEK nicht kalkulationsrelevant sind. Diese werden in der vorliegenden Dissertation über den einheitlichen Bewertungsmaßstab (EBM) der kassenärztlichen Bundesvereinigung beziehungsweise über den Hebammenhilfevertrag abgerechnet.

Der einheitliche Bewertungsmaßstab ist das Vergütungssystem medizinischer Leistungen auf vertragsärztlicher Ebene in Deutschland. Der EBM gilt für die ambulante Versorgung von gesetzlich Versicherten und ordnet jeder Leistung, die ein Arzt/eine Ärztin im Rahmen der medizinischen Behandlung unternimmt, eine Ziffer und Punktzahl zu. Diese Punkte werden dann mit einem kalkulatorischen Punktwert multipliziert. Dieser Punktwert ist allerdings nur eine Orientierung, sofern kein Centwert bereits im jeweiligen EBM festgelegt ist. Der endgültige Centwert des Punktes wird erst nach dem Abrechnungszeitraum festgelegt. Es resultiert dann die abzurechnende Leistung, die von dem Kostenträger erstattet wird. In dieser Dissertation wird von den Orientierungspunktwerten der Jahre 2010 bis 2015 jeweils des ersten Quartals ausgegangen und zwischen spontaner Geburt und primärer Sectio unterschieden. Es werden nur die minimalen, obligaten Leistungen während einer Geburt angegeben (Tabelle 5).

Der Hebammenhilfevertrag ist ein Vertrag zwischen den Hebammenverbänden und dem GKVSpitzenverband, der die Versorgung der Hebammenhilfe laut §134a SGB V regelt. Hierbei werden auch 
die abrechenbaren Leistungen definiert. Diese Leistungen finden sich in der Hebammenvergütungsvereinbarung. Da es sich für diese Dissertation um den zu beobachtenden Zeitraum von 2010 bis 2015 handelt, wurden die abzurechnenden Leistungen entsprechend des letzten, seit 01. Januar 2018 nicht mehr gültigen Hebammenvergütungsverzeichnisses zum Vertrag §134a SGB V, entnommen. Ein Unterschied in der Abrechnung zwischen Spontangeburt und Kaiserschnitt findet sich hier nicht. Es werden nur die minimalen, obligaten Leistungen während einer Geburt angegeben (Tabelle 6).

Tabelle 3: Bewertungsrelationen der DRGs aus den Fallpauschalenkatalogen und Landesbasisfallwerte der Datenjahre 20102016

\begin{tabular}{|c|c|c|c|c|c|c|c|}
\hline & 2010 & 2011 & 2012 & 2013 & 2014 & 2015 & 2016 \\
\hline $\begin{array}{l}\text { Landesbasisfallwert } \\
\text { Schleswig-Holstein } \\
\text { ohne Ausgleiche }\end{array}$ & $2.855,49$ & $2.884,86$ & $2.945,74$ & $3.012,04$ & $3.117,36$ & $3.190,81$ & $3.278,19$ \\
\hline \multicolumn{8}{|l|}{ Belegabteilung } \\
\hline O60D & 0,327 & 0,32 & 0,321 & 0,317 & 0,32 & 0,321 & - \\
\hline $\mathrm{O} 01 \mathrm{H}$ & 0,622 & 0,6 & 0,594 & 0,59 & 0,6 & 0,609 & - \\
\hline O01E & 0,79 & 0,721 & 0,709 & 0,707 & 0,711 & 0,711 & - \\
\hline \multicolumn{8}{|l|}{$\begin{array}{l}\text { Hauptabteilung mit } \\
\text { Beleghebammen }\end{array}$} \\
\hline O60D & 0,412 & 0,413 & 0,435 & 0,433 & 0,44 & 0,449 & 0,449 \\
\hline $\mathrm{O} 01 \mathrm{H}$ & 0,764 & 0,766 & 0,786 & 0,773 & 0,79 & 0,786 & 0,78 \\
\hline O01E & 0,993 & 0,986 & 0,99 & 0,99 & 0,973 & 0,976 & 0,978 \\
\hline \multicolumn{8}{|l|}{$\begin{array}{l}\text { Hauptabteilung mit } \\
\text { angestellten } \\
\text { Hebammen }\end{array}$} \\
\hline O60D & 0,507 & 0,51 & 0,537 & 0,54 & 0,545 & 0,566 & 0,566 \\
\hline $\mathrm{O} 01 \mathrm{H}$ & 0,803 & 0,811 & 0,835 & 0,823 & 0,846 & 0,849 & 0,84 \\
\hline O01E & 1,14 & 1,139 & 1,136 & 1,133 & 1,119 & 1,114 & 1,118 \\
\hline
\end{tabular}


Tabelle 4: Abrechnung einer Geburt nach DRG Entgeltrechnung in Euro

\begin{tabular}{|c|c|c|c|c|c|c|c|}
\hline & 2010 & 2011 & 2012 & 2013 & 2014 & 2015 & 2016 \\
\hline \multicolumn{8}{|c|}{ Belegabteilung } \\
\hline O60D & 933,75 & 923,16 & 945,58 & 954,82 & 997,56 & 1024,25 & - \\
\hline $\mathrm{O} 01 \mathrm{H}$ & $1.776,11$ & $1.730,92$ & $1.749,77$ & $1.777,10$ & $1.870,42$ & $1.943,20$ & - \\
\hline O01E & $2.255,84$ & $2.079,98$ & $2.088,53$ & $2.129,51$ & $2.216,44$ & $2.268,67$ & - \\
\hline \multicolumn{8}{|c|}{$\begin{array}{l}\text { Hauptabteilung } \\
\text { mit } \\
\text { Beleghebammen }\end{array}$} \\
\hline O60D & $1.176,46$ & $1.191,48$ & $1.281,4$ & $1.304,21$ & $1.371,64$ & $1.432,67$ & $1.471,91$ \\
\hline $\mathrm{O} 01 \mathrm{H}$ & $2.181,59$ & $2.209,80$ & $2.315,35$ & $2.328,31$ & $2.462,71$ & $2.507,98$ & $2.556,99$ \\
\hline O01E & $2.835,50$ & $2.844,47$ & $2.916,28$ & $2.981,92$ & $3.033,19$ & $3.114,23$ & $3.206,07$ \\
\hline \multicolumn{8}{|c|}{$\begin{array}{l}\text { Hauptabteilung } \\
\text { mit angestellten } \\
\text { Hebammen }\end{array}$} \\
\hline O60D & $1.447,73$ & $1.471,28$ & $1.581,86$ & $1.626,50$ & $1.698,96$ & 1.806 & $1.855,46$ \\
\hline $\mathrm{O} 01 \mathrm{H}$ & $2.292,96$ & $2.339,62$ & $2.459,69$ & $2.478,91$ & $2.637,29$ & 2.708 & $2.753,68$ \\
\hline O01E & $3.255,26$ & $3.285,86$ & $3.346,36$ & $3.412,64$ & $3.488,33$ & $3.554,56$ & $3.665,02$ \\
\hline
\end{tabular}

Tabelle 5: Abrechnung einer Geburt in Euro nach dem einheitlichen Bewertungsmaßstab (EBM) der Jahre 2010-2015

\begin{tabular}{|l|l|r|r|r|r|r|r|}
\hline & & $\mathbf{2 0 1 0}$ & $\mathbf{2 0 1 1}$ & $\mathbf{2 0 1 2}$ & $\mathbf{2 0 1 3}$ & $\mathbf{2 0 1 4}$ & $\mathbf{2 0 1 5}$ \\
\hline 08211 & Grundpauschale & 14,72 & 14,37 & 14,37 & 14,50 & 14,69 & 14,89 \\
\hline 08231 & $\begin{array}{l}\text { Zuschlag zur Grundpauschale } \\
\text { im Rahmen der Geburtshilfe, } \\
\text { bei denen } \\
\text { Gebührenordnungspositionen } \\
\text { des Abschnitts 8.4 berechnet } \\
\text { werden }\end{array}$ & 8,94 & 8,94 & 8,94 & 9,02 & 9,12 & 9,24 \\
\hline 08410 & $\begin{array}{l}\text { Verweilen im Gebärraum } \\
\text { ohne Erbringung weiterer } \\
\text { berechnungsfähiger } \\
\text { Leistungen, wegen der } \\
\text { Betreuung einer Geburt } \\
\text { erforderlich, je vollendete } 30 \\
\text { Minuten }\end{array}$ & 24,36 & 24,36 & 24,36 & 24,58 & 24,92 & 25,27 \\
\hline 08411 & $\begin{array}{l}\text { Betreuung und Leitung einer } \\
\text { Geburt }\end{array}$ & 126,17 & 201,18 & 201,18 & 202,98 & 205,64 & 208,52 \\
\hline 08415 & $\begin{array}{l}\text { Zuschlag zur } \\
\text { Gebührenordnungsposition } \\
\text { o8411 bei Leitung und } \\
\text { Betreuung einer Geburt bei } \\
\text { Schnittentbindung }\end{array}$ & 68,34 & 68,34 & 68,34 & 68,96 & 69,90 & 70,88 \\
\hline Gesamt Spontangeburt & 174,19 & 248,84 & 248,84 & 251,08 & 254,37 & 257,92 \\
\hline Gesamt primäre Sectio & 242,53 & 317,18 & 317,18 & 320,04 & 324,27 & 328,80 \\
\hline
\end{tabular}


Tabelle 6: Abrechnung einer Geburt in Euro nach der Hebammen-Vergütungsvereinbarung

\begin{tabular}{|l|l|r|}
\hline Ziffer & Leistung & Gebühr in Euro \\
\hline 090 & Hilfe bei der Geburt eines Kindes in einem Krankenhaus & 224,40 \\
\hline 320 & $\begin{array}{l}\text { Wegegeld bei einer Entfernung von mehr als zwei } \\
\text { Kilometern zwischen der Wohnung oder Praxis der } \\
\text { Hebamme und der Stelle der Leistung, für jeden } \\
\text { zurückgelegten Kilometer (Annahme 10km) }\end{array}$ & 5,90 \\
\hline 360 & Materialpauschale Geburtshilfe & 14,69 \\
\hline 260 & Überwachung, je angefangene halbe Stunde & 7,65 \\
\hline 240 & $\begin{array}{l}\text { Erstuntersuchung des Kindes einschließlich Eintragung } \\
\text { der Befunde in das Untersuchungsheft für Kinder (U 1) } \\
\text { nach den Richtlinien des Bundesausschusses der Ärzte } \\
\text { und Krankenkassen über die Früherkennung von } \\
\text { Krankheiten bei Kindern bis zur Vollendung des 6. } \\
\text { Lebensjahres (Kinder-Richtlinien) in der jeweils } \\
\text { geltenden Fassung }\end{array}$ & 286,64 \\
\hline Gesamt & & \\
\hline
\end{tabular}




\subsubsection{Fallzahlen Klinikum Nordfriesland gGmbH, Standort Wyk/ Föhr}

Die Klinikum Nordfriesland gGmbh hat ihren Sitz in Husum und Standorte in Niebüll, Tönning und Wyk auf Föhr. Krankenhausträger ist zu 100\% der Kreis Nordfriesland. Zum Berichtsjahr 2015 verfügte das Krankenhaus Nordfriesland über 387 vollstationäre Planbetten in den Festlandkliniken, sowie 18 vollstationäre Betten in der Inselklinik Wyk auf Föhr. Für das Budget 2015 konnte ein Sicherstellungszuschlag für die Inselklinik Wyk auf Föhr in Höhe von 2.150.000 Euro ausgehandelt werden. Das Klinikum Nordfriesland betreibt weiterhin vier Tochtergesellschaften. Die für die Dissertation relevanten Zahlen sind den veröffentlichten Qualitätsberichten auf Grundlage von 137 Abs. 3 Satz 1 Nr. 4 SGB V entnommen und in Tabelle sieben und acht abgebildet. Für das Berichtsjahr 2011 ist kein Qualitätsbericht erschienen, da erst ab dem Jahr 2012 eine jährliche Pflicht zur Veröffentlichung bestand.

Tabelle 7: Fallzahlen Klinikum Nordfriesland gGmbH, Inselklinik Föhr/ Amrum

\begin{tabular}{|l|r|r|r|r|r|}
\hline & $\mathbf{2 0 1 0}$ & $\mathbf{2 0 1 2}$ & $\mathbf{2 0 1 3}$ & $\mathbf{2 0 1 4}$ & $\mathbf{2 0 1 5}$ \\
\hline Anzahl Betten & 18 & 18 & 18 & 18 & 18 \\
\hline Fallzahlen vollstationär & 1.543 & 1.543 & 1.446 & 1.362 & 1.332 \\
\hline Ärzte/Ärztinnen gesamt & 8 & 8,1 & 7,9 & 6,5 & 5,63 \\
\hline Fachärzte (Vollkräfte) & 4 & 4,3 & 4,2 & 3,3 & 3 \\
\hline Belegärzte & 1 & 1 & 1 & 1 & 1 \\
\hline
\end{tabular}

Ferner wurden die Fallzahlen der Geburtshilfe der Inselklinik Föhr/Amrum aus den Qualitätsberichten entnommen. Hierbei ist anzumerken, dass Fallzahlen kleiner als vier aufgrund datenschutzrechtlicher Aspekte nicht in den Qualitätsberichten abgebildet werden.

Tabelle 8: Fallzahlen der geburtshilflichen DRGs in der Inselklinik Föhr/Amrum 2010-2015. Quelle: Qualitätsreporte Klinikum Nordfriesland gGmbH, Inselklinik Föhr/Amrum 2010-2015

\begin{tabular}{|l|l|r|r|r|r|r|}
\hline \multicolumn{2}{|l|}{ Diagnosen/DRG } & $\mathbf{2 0 1 0}$ & $\mathbf{2 0 1 2}$ & $\mathbf{2 0 1 3}$ & $\mathbf{2 0 1 4}$ & $\mathbf{2 0 1 5}$ \\
\hline Z38.0 (ICD-10) & 64 & 45 & 58 & 54 & 40 \\
\hline DRG & Code & \multicolumn{5}{|l|}{} \\
\hline O60D & O80 & 31 & 13 & 17 & 39 & 19 \\
\hline O60D & O81 & & & & 4 & 14 \\
\hline O01H & O82 & 15 & 12 & 20 & 18 & 14 \\
\hline
\end{tabular}


Parallel dazu konnte Einsicht in die Unterlagen einer auf Föhr arbeitenden Hebamme genommen werden, die alle Geburten, die in der geburtshilflichen Abteilung des Krankenhauses auf Föhr zwischen 2011 und 2015 stattgefunden haben, archiviert hat. Hausgeburten werden nicht berücksichtigt.

Tabelle 9: Fallzahlen der geburtshilflichen DRGs aus persönlicher Quelle einer Föhrer Hebamme. Quelle: Persönliche Angaben einer Föhrer Hebamme

\begin{tabular}{|c|c|c|c|c|c|c|}
\hline \multicolumn{2}{|c|}{ Diagnosen/DRG } & 2011 & 2012 & 2013 & 2014 & 2015 \\
\hline \multicolumn{2}{|c|}{ Z38.0 (ICD-10) } & 49 & 47 & 58 & 64 & 45 \\
\hline DRG & Code & & & & & \\
\hline O60D & 080 & 35 & 33 & 33 & 40 & 19 \\
\hline O60D & 081 & & & 2 & 4 & 4 \\
\hline $\mathrm{O} 01 \mathrm{H}$ & 082 & 6 & 8 & 8 & 10 & 13 \\
\hline \multicolumn{2}{|l|}{ O01E } & 8 & 6 & 15 & 10 & 9 \\
\hline
\end{tabular}

Erklärungen zu den Tabellen 8 und 9:

Z38: Einling, Geburt im Krankenhaus (ICD-10)

O01E: Sekundäre Sectio caesarea mit komplizierender Diagnose, Schwangerschaftsdauer mehr als 33 vollendete Wochen (SSW), ohne komplexe Diagnose

O01H: Primäre Sectio caesarea ohne komplizierende Diagnose, Schwangerschaftsdauer mehr als 33 vollendete Wochen (SSW), ohne komplexe Diagnose

O60D: Vaginale Entbindung ohne komplizierende Diagnose

O80: Spontangeburt eines Einlings

081: Geburt eines Einlings durch Zangen- oder Vakuumextraktion

082: Geburt eines Einlings durch Schnittentbindung

Es fallen Abweichungen in den Angaben zu den jährlichen Geburtenfallzahlen auf. Dies mag zum Teil darin begründet sein, dass in den Qualitätsberichten aus Datenschutzgründen Fallzahlen kleiner vier nicht abgebildet sind. Ein Vergleich mit den Daten des Ministeriums für Soziales, Gesundheit, Jugend und Senioren [18] macht deutlich, dass es keine vollständige Übereinstimmung zwischen den drei genannten Quellen gibt. 
Tabelle 10: Geburten im Krankenhaus "Inselklinik Föhr/Amrum", entnommen aus der Drucksache 13-3338 des MSGFGs

\begin{tabular}{|l|r|r|r|r|r|}
\hline & 2010 & 2011 & 2012 & 2013 & 2014 \\
\hline $\begin{array}{l}\text { Anzahl Geburten im Krankenhaus Inselklinik } \\
\text { Föhr/Amrum }\end{array}$ & 64 & 48 & 45 & 57 & 64 \\
\hline
\end{tabular}

Zudem kann das Jahr 2015 nur bedingt betrachtet werden, da die Schließung der Geburtshilfe auf Föhr bereits zum 01. Oktober 2015 erfolgte. Für die weiteren Berechnungen im Rahmen der vorliegenden Dissertation wurden die Angaben der Hebamme zu den Geburtsfallzahlen übernommen, da diese 2011 und 2013 nur eine Abweichung von einer Geburt und 2012 von zwei Geburten im Vergleich zu den Daten des MSGFGs zeigen. Die Geburtsfallzahlen aus den Qualitätsberichten zeigen eine deutlich höhere Abweichung, vor allem im Jahr 2014. Zudem ist durch die Angaben der Hebamme eine genaue Aufschlüsselung der Geburtsmodi möglich ist. Für das Jahr 2010 werden die Angaben des MSGFGs übernommen. 


\subsubsection{Erfassung der Kosten einer Geburt in einer Belegabteilung, in einer Hauptabteilung mit}

\section{Beleghebammen sowie einer Hauptabteilung mit angestellten Hebammen}

Auf Basis der vorhergehenden Kapitel können nun für alle drei Abteilungsarten in der Geburtshilfe die Kosten einer Geburt aus Kostenträgerperspektive errechnet werden. Die belegärztliche Tätigkeit bei primärer und sekundärer Sectio wird mit der gleichen Gebühr verrechnet.

Tabelle 11: Kosten einer Geburt in Euro in einer Belegabteilung

\begin{tabular}{|l|r|r|r|r|r|r|}
\hline & $\mathbf{2 0 1 0}$ & $\mathbf{2 0 1 1}$ & $\mathbf{2 0 1 2}$ & $\mathbf{2 0 1 3}$ & $\mathbf{2 0 1 4}$ & $\mathbf{2 0 1 5}$ \\
\hline O60D & 933,75 & 923,16 & 945,58 & 954,82 & 997,56 & 1024,25 \\
\hline O01H & $1.776,11$ & $1.730,92$ & $1.749,77$ & $1.777,10$ & $1.870,42$ & $1.943,20$ \\
\hline O01E & $2.255,84$ & $2.079,98$ & $2.088,53$ & $2.129,51$ & $2.216,44$ & $2.268,67$ \\
\hline $\begin{array}{l}\text { Belegärztliche } \\
\text { Tätigkeiten } \\
\text { Spontangeburt }\end{array}$ & 174,19 & 248,84 & 248,84 & 251,08 & 254,37 & 257,92 \\
\hline $\begin{array}{l}\text { Belegärztliche } \\
\text { Tätigkeiten } \\
\text { Primäre Sectio }\end{array}$ & 242,53 & 317,18 & 317,18 & 320,04 & 324,27 & 328,80 \\
\hline $\begin{array}{l}\text { Gebühr } \\
\text { Beleghebamme }\end{array}$ & 286,64 & 286,64 & 286,64 & 286,64 & 286,64 & 286,64 \\
\hline \hline $\begin{array}{l}\text { Gesamt } \\
\text { Spontangeburt }\end{array}$ & $1.394,58$ & $1.458,64$ & $1.481,06$ & $1.492,54$ & $1.538,57$ & $1.568,81$ \\
\hline $\begin{array}{l}\text { Gesamt primäre } \\
\text { Sectio }\end{array}$ & $2.305,28$ & $2.334,74$ & $2.353,59$ & $2.383,78$ & $2.481,33$ & $2.558,64$ \\
\hline $\begin{array}{l}\text { Gesamt sekundäre } \\
\text { Sectio }\end{array}$ & $2.785,01$ & $2.683,8$ & $2.692,35$ & $2.736,19$ & $2.827,35$ & $2.884,11$ \\
\hline
\end{tabular}


Tabelle 12: Kosten einer Geburt in Euro in einer Hauptabteilung mit Beleghebammen

\begin{tabular}{|l|r|r|r|r|r|r|r|}
\hline & $\mathbf{2 0 1 0}$ & $\mathbf{2 0 1 1}$ & $\mathbf{2 0 1 2}$ & $\mathbf{2 0 1 3}$ & $\mathbf{2 0 1 4}$ & $\mathbf{2 0 1 5}$ & $\mathbf{2 0 1 6}$ \\
\hline $\mathrm{O} 60 \mathrm{D}$ & $1.176,46$ & $1.191,48$ & $1.281,4$ & $1.304,21$ & $1.371,64$ & $1.432,67$ & $1.471,91$ \\
\hline $\mathrm{O} 01 \mathrm{H}$ & 2181,59 & $2.209,80$ & $2.315,35$ & $2.328,31$ & $2.462,71$ & $2.507,98$ & $2.556,99$ \\
\hline O01E & $2.835,50$ & $2.844,47$ & $2.916,28$ & $2.981,92$ & $3.033,19$ & $3.114,23$ & $3.206,07$ \\
\hline $\begin{array}{l}\text { Gebühr } \\
\text { Beleghebamme }\end{array}$ & 286,64 & 286,64 & 286,64 & 286,64 & 286,64 & 286,64 & 286,64 \\
\hline $\begin{array}{l}\text { Gesamt } \\
\text { Spontangeburt }\end{array}$ & $1.463,1$ & $1.478,12$ & $1.568,04$ & $1.590,85$ & $1.658,28$ & $1.719,31$ & $1.758,55$ \\
\hline $\begin{array}{l}\text { Gesamt primäre } \\
\text { Sectio }\end{array}$ & $2.468,23$ & $2.496,44$ & $2.601,99$ & $2.614,95$ & $2.749,35$ & $2.794,62$ & $2.843,63$ \\
\hline $\begin{array}{l}\text { Gesamt sekundäre } \\
\text { Sectio }\end{array}$ & $3.122,14$ & $3.131,11$ & $3.202,92$ & $3.268,56$ & $3.319,83$ & $3.400,87$ & $3.492,71$ \\
\hline
\end{tabular}

Tabelle 13: Kosten einer Geburt in Euro in einer Hauptabteilung

\begin{tabular}{|l|r|r|r|r|r|r|r|}
\hline & $\mathbf{2 0 1 0}$ & $\mathbf{2 0 1 1}$ & $\mathbf{2 0 1 2}$ & $\mathbf{2 0 1 3}$ & $\mathbf{2 0 1 4}$ & $\mathbf{2 0 1 5}$ & $\mathbf{2 0 1 6}$ \\
\hline $\begin{array}{l}\text { Gesamt } \\
\text { Spontangeburt }\end{array}$ & $1.447,73$ & $1.471,28$ & $1.581,86$ & $1.626,50$ & $1.698,96$ & 1.806 & $1.855,46$ \\
\hline $\begin{array}{l}\text { Gesamt primäre } \\
\text { Sectio }\end{array}$ & $2.292,96$ & $2.339,62$ & $2.459,69$ & $2.478,91$ & $2.637,29$ & 2.708 & $2.753,68$ \\
\hline $\begin{array}{l}\text { Gesamt sekundäre } \\
\text { Sectio }\end{array}$ & $3.255,26$ & $3.285,86$ & $3.346,36$ & $3.412,64$ & $3.488,33$ & $3.554,56$ & $3.665,02$ \\
\hline
\end{tabular}




\subsubsection{Messung der Ressourcenverbräuche und monetäre Bewertung der}

\section{Ressourcenverbräuche}

Zur Messung der Ressourcenverbräuche wurde ein "top-down orientierter macro-costing Ansatz“ [40] gewählt, da für viele Kostenkategorien nur Durchschnittskosten zur Verfügung standen und eine differenzierte Auflistung der Einzelkosten nicht möglich war. Eine potenzielle Zusammenarbeit mit dem Klinikum Nordfriesland Standort Wyk kam aufgrund der gesundheitspolitischen Lage nicht in Frage und auch unser Kooperationspartner vor Ort konnte uns über die konkreten Einzelkosten des Kreißsaals, als dieser noch in Betrieb war, keine Auskunft geben. Die Benutzung von Durchschnittswerten für die Messung des Ressourcenverbrauchs, wenn andere Möglichkeiten zur Einzelkostenerfassung nicht möglich sind, ist explizit durch die deutschen Empfehlungen zur gesundheitsökonomischen Evaluation, dem Hannoveraner Konsens, erlaubt [44].

Die direkten medizinischen Kosten konnten so über die Gebührenordnungen im Rahmen der DRGs, der Hebammenvergütungsvereinbarung sowie des EBM der Kassenärztlichen Bundesvereinigung (KBV) erfasst werden. Diese Gebührenordnungen spiegeln zwar nicht den Ressourceneinsatz wider, zeigen aber die realen Kosten, die den Kostenträgern, in diesem Fall den gesetzlichen Krankenkassen, entstehen. Daher ist es akzeptabel, mit den Kostensätzen nach DRG, EBM und Hebammenvergütungsvereinbarung zu arbeiten, da diese budgetrelevant für die Kostenträger sind.

Ebenso verhält es sich mit den direkten nicht-medizinischen Kosten für die Haushalte. Anhand der Ergebnisse der Online-Umfrage kann man durchschnittliche Angaben zur Häufigkeit von Fahrten, Verteilung der Unterkünfte und auch Dauer des Aufenthalts in der Boarding Einrichtung erhalten. Außerdem wurden die Frauen gebeten, quantitative Angaben zu den drei Kostenkomponenten Transport, Verpflegung und Unterkunft zu machen. Hieraus wurden Durchschnittswerte gebildet. Diese Durchschnittswerte spiegeln die Kosten aus Perspektive der Studienteilnehmerinnen wider. Wenn die Studienteilnehmerinnen angaben, bei der Familie oder bei Freunden auf dem Festland gewohnt zu haben, wurde gemäß § 9 des Einkommenssteuergesetzes ein Pauschbetrag von 24 Euro pro 24 Stunden angenommen.

Parallel dazu wurde eine Beispielrechnung durchgeführt, die ein Minimum an den entstandenen Kosten für die Föhrer Familien annimmt, um eine Überschätzung der Kosten zu vermeiden [44]. Folgende Annahmen wurden getroffen:

- Es erfolgt eine Fahrt mit dem PKW von der Insel Föhr zur Entbindungsklinik.

- Der prästationäre Aufenthalt dauert zwölf Tage.

- Der stationäre Aufenthalt dauert zwei Tage.

- Es erfolgt eine Fahrt mit dem PKW von der Entbindungsklinik auf die Insel Föhr. 
- Für die Kosten der Fähre wird der PKW Tarif 2018 der Wyker Dampfschiffs-Reederei FöhrAmrum GmbH gewählt. Dieser PKW Tarif wird nach Länge berechnet. Der Tarif beträgt für zwei Personen sowie einen Kleinwagen (Volkswagen Golf) 64,35 Euro für eine Überfahrt. Registrierten Insulanern wird ein „Insulanertarif“ angeboten, der 50\% Nachlass auf den gewählten Tarif gewährt [45].

- Für die Fahrtkosten wird eine Kilometerpauschale von 0,30 Euro angesetzt entsprechend der Entfernungspauschale 2018 nach dem deutschen Einkommenssteuerrecht [46].

- Die Parkplatzkosten werden mit fünf Euro pro 24 Stunden angegeben.

- Die Kilometeranzahl wird ab Dagebüll zu den entsprechenden Entbindungskliniken mit Google Maps (siehe Anhang III) berechnet.

- Es wurden exemplarisch nur die Entbindungskliniken in Flensburg und Heide berücksichtigt.

Tabelle 14: Minimale Transportkosten einer Geburt auf dem Festland, wenn die Eltern Föhrer sind

\begin{tabular}{|l|l|l|l|l|l|}
\hline & Fähre & Kilometer & Fahrtkosten & $\begin{array}{l}\text { Parkkosten } \\
\text { für 14 Tage }\end{array}$ & Gesamt \\
\hline $\begin{array}{l}\text { Fahrt zur Geburt DIAKO } \\
\begin{array}{l}\text { Flensburg } \\
\text { hin und zurück }\end{array}\end{array}$ & $64,35 €$ & 56,8 & $34,08 €$ & $70 €$ & $168,43 €$ \\
\hline $\begin{array}{l}\text { Fahrt zur Geburt WKK Heide } \\
\text { hin und zurück }\end{array}$ & $64,35 €$ & 84,7 & $50,82 €$ & $70 €$ & $185,17 €$ \\
\hline
\end{tabular}

Hierbei ist anzumerken, dass diese Berechnung nur das Minimum an Kosten aus der Perspektive der Föhrer Frauen anzeigt. Zusätzliche Verpflegungs- und Unterbringungskosten wurden nicht berechnet. Zudem werden mögliche Rückerstattungen des Fahrtgeldes der Krankenversicherung nicht berücksichtigt. Erfahrungen aus den Interviews und der Auswertung der Online Umfrage zeigen jedoch, dass dennoch durch verschiedene Faktoren erheblich mehr Kosten entstanden sind. Nähere Erläuterungen dazu erfolgen im Ergebnis- und im Diskussionsteil.

Die Messung der Ressourcenverbräuche der direkten nicht-medizinischen Kosten für das Gesundheitssystem wurde ebenfalls mit Durchschnittswerten bewertet. Hier sei angemerkt, dass es durch fehlende Transparenz bei den Interessenvertretern sehr schwierig war, überhaupt konkrete Angaben über Ressourcenverbräuche zu erhalten.

Folgende Annahmen wurden dabei getroffen: 
Der Rettungstransport einer schwangeren Frau von der Insel Föhr auf das Festland kann auf drei verschiedenen Wegen erfolgen: per Rettungswagen, per Seenotrettungskreuzer oder per Luftrettung mit einem Helikopter.

Die Kosten des Rettungswagens werden durch eine Pauschale und ein Entgelt pro Beförderungskilometer berechnet. Grundlage dafür ist die „Vereinbarung über Benutzungsentgelte für die Leistungen des Rettungsdienstes gemäß Ziffer 6.2. der Vereinbarung von Eckpunkten zur Umsetzung der Vereinbarungslösung im Rettungsdienst Schleswig-Holstein nach §8a des Rettungsdienstgesetzes zwischen dem Kreis Nordfriesland als Träger des Rettungsdiensts sowie den gesetzlichen und privaten Krankenkassen als Kostenträger" [47]. Es wurde weiterhin festgelegt, dass es sich bei einem Schwangerentransport von der Insel immer um einen Notfall handelt und daher ein Rettungstransport in die Kostenberechnung eingeht und kein Krankentransport. Die Pauschale für den Rettungstransport mit dem Rettungswagen beträgt 797,03 Euro, das Entgelt pro Beförderungskilometer 6,76 Euro. Die Distanzen von Dagebüll zu den einzelnen, relevanten Entbindungskliniken wurden durch Berechnungen mittels Google Maps (kürzeste Strecke) erhoben.

Der Seenotrettungskreuzer wird von der „Deutschen Gesellschaft zur Rettung Schiffbrüchiger (DGzRS)“ betrieben und ist in Amrum stationiert. Ein Einsatz wird pauschal pro Stunde abgerechnet. Diese Pauschale beträgt circa 300 Euro pro Stunde. [48] Der Seenotrettungskreuzer muss nach Benachrichtigung erst von Amrum nach Föhr, dann nach Dagebüll und wieder nach Amrum zurückfahren. Hinzu kommt die Zeit zur Be- und Entladung der Schwangeren. Es wird daher angenommen, dass ein Einsatz zwei Stunden dauert.

Die Luftrettung erfolgt durch die DRF-Luftrettung (Deutsche Rettungsflugwacht e.V.). Helikopter, die Föhr anfliegen, sind in Niebüll und Rendsburg stationiert. Von Niebüll beträgt die reine Flugzeit nach Föhr unter fünfzehn Minuten. Es wird daher angenommen, dass ein kompletter Einsatz mit Flugzeit nach Föhr, Schwangerenrettung auf Föhr, Schwangerentransport auf das Festland zurück und Übergabe der Schwangeren an die entsprechende Entbindungsklinik eine Stunde in Anspruch nimmt. Laut Presseberichten wird die Flugzeit pauschal mit 45 Euro pro Minute abgerechnet [49]. Konkretere Angaben von offiziellen Stellen der DRF über die Einsatzzeit und Pauschale wurden bei der Recherche leider verweigert.

Die Unterbringung der schwangeren Frauen auf Föhr erfolgt in kliniknahen Boarding-Häusern oder Ferienwohnungen. Die durchschnittliche Aufenthaltsdauer in solch einer Einrichtung können wir der durchgeführten Umfrage entnehmen. Nach ausführlicher Recherche erhielt man die Information, dass diese Unterbringungskosten als kostendeckender Pauschalbetrag in die Krankenhausbudgets nach $\S 18$ KHG eingerechnet werden. Der Grund dafür ist, dass es sich für die Krankenhäuser um Vorhaltekosten 
und Aufenthaltskosten, nicht aber um Behandlungskosten handelt [50]. Diese kostendeckenden Pauschalbeträge weichen dementsprechend auch von Krankenhaus zu Krankenhaus ab. Die verschiedenen Pauschalbeträge wurden auch nach intensiven Gesprächen mit dem Verband deutscher Ersatzkassen sowie anderen Versicherungen nicht mitgeteilt. Für diese Dissertation wird daher angenommen, dass eine Unterbringung in einem Boarding-Haus pro Nacht durchschnittlich 35 Euro kostet. Dies ist laut Auswertung der Umfragebögen der Betrag, den der Partner für eine weitere Unterbringung in dem Boarding-Haus nach der Geburt des Kindes selbst zahlen muss. Dieses Szenario trifft ein, wenn in der Klinik nach der Geburt kein Familienzimmer frei ist.

Nach Schließung der stationären Geburtshilfe auf Föhr wurde die Versorgung der schwangeren Frauen durch einen Hebammennotruf sichergestellt, an dem zunächst zwei der drei Hebammen von Föhr/Amrum teilnahmen. Der Notruf war an 365 Tagen pro Jahr erreichbar und wurde Anfang 2016 installiert. Die Hebammen erhielten 45 Euro pro 24 Stunden Rufbereitschaft. Die 45 Euro wurden aus freiwilligen Mitteln des Kreises Nordfriesland getragen. Die Krankenkassen finanzierten den Notruf mit einem Maximum von 9.600 Euro pro Jahr mit. Weiterhin trat der Kreis Nordfriesland in Vorleistung und übernahm die Versicherungsbeiträge der zwei an dem Notruf teilnehmenden Hebammen mit insgesamt 14.463,48 Euro pro Jahr [51]. Diese Art der Finanzierung des Notrufs wurde zum 01. Januar 2018 umgestellt [52] und der Finanzierung des Notrufs der Insel Sylt angepasst. Für diese Dissertation wird jedoch die aufgezeigte Finanzierung des Hebammennotrufs beleuchtet, da sie für den Zeitraum der Studie in Kraft war.

Zusätzlich übernimmt der Kreis Nordfriesland die Förderung des Rufdienstes der durchführenden Fachärztin für Frauenheilkunde und Geburtshilfe in Höhe von 131 Euro pro 24-Stunden-Rufdienst sowie einen Anteil von 5.820,75 Euro an der Haftpflichtversicherung für den Zeitraum vom 01. April 2017 bis zum 31.12.2017 (275 Ruftage) [53]. Für den Zeitraum nach der Schließung bis zum 31. März 2017 übernimmt das Krankenhaus Nordfriesland diese Rufdienstpauschale (90 Ruftage im Jahr 2017). 
Tabelle 15: Verbrauch und monetäre Bewertung der Ressourcen

\begin{tabular}{|c|c|c|c|c|c|}
\hline \multirow[t]{2}{*}{ Ressource } & \multicolumn{2}{|c|}{ Ressourcenverbrauch } & \multicolumn{2}{|c|}{ Monetäre Bewertung der Ressource } & \multirow[t]{2}{*}{ Kostenträger } \\
\hline & & & Pauschalentgelt & $\begin{array}{r}\text { Entgelt } \\
\begin{array}{r}\text { Beförderungskilometer/- } \\
\text { stunde/-minute/-tag }\end{array}\end{array}$ & \\
\hline \multirow[t]{2}{*}{ Rettungswagen } & $\begin{array}{r}\text { DIAKO } \\
\text { Flensburg }\end{array}$ & $56,8 \mathrm{~km}$ & $797,03 €$ & 6,76€/ Kilometer & Krankenversicherung \\
\hline & $\begin{array}{l}\text { WKK } \\
\text { Heide }\end{array}$ & $84,7 \mathrm{~km}$ & & & \\
\hline $\begin{array}{l}\text { Seenotrettungs- } \\
\text { kreuzer }\end{array}$ & \multicolumn{2}{|c|}{2 Stunden pro Einsatz } & & $300 € /$ Stunde & Krankenversicherung \\
\hline Luftrettung & \multicolumn{2}{|c|}{1 Stunden pro Einsatz } & & $45 € /$ Minute & Krankenversicherung \\
\hline Boarding & & & & $35 € /$ Tag & Krankenversicherung \\
\hline Hebammennotruf & & 365 Tage & & $45 € /$ Tag & Kreis NF \\
\hline Hebammennotruf & & 365 Tage & $9600 €$ & & Krankenversicherung \\
\hline $\begin{array}{l}\text { Versicherung } \\
\text { Hebamme }\end{array}$ & $2 \mathrm{H}$ & bammen & $7181,74 €$ & & Kreis NF \\
\hline Rufdienst Gynäkologin & $1 \mathrm{~Gy}$ & läkologin & & $131 € /$ Tag & $\begin{array}{r}\text { Kreis NF (ab 01.April } \\
2017) \\
\text { Klinikum NF (bis } \\
31.03 .2017) \\
\end{array}$ \\
\hline $\begin{array}{l}\text { Versicherung } \\
\text { Gynäkologin }\end{array}$ & $1 \mathrm{~Gy}$ & läkologin & $5820,75 €$ & & Kreis NF \\
\hline
\end{tabular}




\subsection{Datenerhebung mittels Interviews und Online-Befragung}

\subsubsection{Vorarbeiten und Durchführung der qualitativen Interviews}

Zur Vorarbeit im Rahmen der Dissertation wurde eine umfassende Literatur- und eine Medienrecherche betrieben, in der speziell die Umstände und chronologischen Abläufe der Schließung der Geburtshilfestation auf Föhr identifiziert wurden. Die Abläufe der Schließung und der geburtshilflichen Situation wurden schon in der Einleitung näher beleuchtet.

Die Literaturrecherche beinhaltete vor allem die Suche nach vergleichbaren Studien, in deren Rahmen ebenfalls Interviews oder Fragebögen zu Geburtserfahrungen von Müttern erstellt worden waren. Eine gute Übersicht bot dabei das Review „Measuring women's childbirth experiences" von Nilvér [54]. Die Interviews sollten so offen wie möglich gestaltet werden, jedoch aber ein für alle Interviews reproduzierbares Rahmengerüst enthalten. Daher fiel die Entscheidung für ein qualitatives, leitfadengestütztes Interview. Anhand des 4 Schritte Prinzips von Helfferich [55] wurde nach den Vorarbeiten ein Interviewleitfaden erarbeitet. Die vier Schritte beinhalten das Sammeln von Fragen, das Prüfen der gesammelten Fragen in Hinblick auf eine weitere Reduzierung, das Sortieren der Fragen nach zeitlichen und inhaltlichen Aspekten sowie das Zusammenfassen der Aspekte zu Erzählaufforderungen [55]. Für den qualitativen Teil wurden semi-strukturierte Interviews, die sich an dem Interviewleitfaden orientierten, durchgeführt. Dieser wurde benutzt, um ein episodisches Interview [56, 57] mit den Teilnehmerinnen durchzuführen, in dem die Erfahrungen der Teilnehmerinnen rund um die Geburt ihres Kindes auf dem Festland erfasst wurden. Das Studiendesign wurde mit der Durchführung von qualitativen Interviews begonnen, da zu dieser expliziten Fragestellung noch keine Daten erhoben wurden. So musste man zunächst explorativ vorgehen und eine „Alltagswirklichkeit des Untersuchungsobjekts“ finden [56]. Hierbei ist immer die Gefahr, dass der Beobachter, in diesem Fall der Interviewer, die Wahrheit subjektiv verzerrt. Um diese Verzerrung zu minimieren wurde ein Interviewleitfaden benutzt.

Der Interviewleitfaden für die Interviews im Rahmen dieser Dissertation ist im Anhang (Anhang I) aufgeführt.

\subsubsection{Rekrutierung und Einschlusskriterien der Teilnehmerinnen der Interviews}

Die Teilnehmerinnen der Interviews waren Frauen auf Föhr, die nach der Schließung der Geburtshilfestation zum 01. Oktober 2015 ein Kind auf dem Festland bekommen haben. Hierbei geht es stets um das erste Kind, das nach der Schließung der Geburtshilfestation geboren wurde. Die Teilnehmerinnen wurden von unserem Kooperationspartner vor Ort, dem Inselgeburt e.V., durch einen allgemeinen Aufruf rekrutiert. An jeweils zwei Tagen im März 2017 und im April 2017 wurden insgesamt 19 Frauen in Einzelinterviews persönlich befragt. Die Teilnehmerinnen wurden über die 
Teilnahme an der Studie aufgeklärt. Die Einverständniserklärungen der Teilnehmerinnen der Interviews liegen dem Institut für Allgemeinmedizin, Universität zu Kiel, vor (Anhang V).

\subsubsection{Auswertung und Ergebnisse der qualitativen Interviews}

Die Interviews hatten eine Durchschnittsdauer von 34 Minuten und 51 Sekunden und wurden, um eine erhöhte Validität in der Auswertung zu erhalten, mit einem digitalen Tonaufnahmegerät aufgezeichnet. Die Interviews wurden von drei Interviewern unabhängig voneinander durchgeführt.

Anschließend wurden die Interviews mit MAXQDA 12 transkribiert und eine qualitative Inhaltsanalyse [38] vorgenommen. Die Transkription erfolgte von zwei voneinander unabhängigen Promotionsstudentinnen. Es wurden keine sprachlichen Feinheiten, wie etwa Dialekte, transkribiert, da die Auswertung der Interviews auf den inhaltlichen Aussagen, nicht den sprachlichen Feinheiten der Teilnehmerinnen beruhte. Darauffolgend wurde eine zusammenfassende Inhaltsanalyse durchgeführt mit dem Ziel, das Material systematisch auf das Wesentliche zu reduzieren (Mayring, 2010). So wurde induktiv ein Kategoriensystem erarbeitet, das schließlich in einem ersten Codebaum resultierte. Neu aufkommende Kategorien wurden bei Analyse der Interviews in das Coding System übernommen (Abbildung 5). Um die Gütekriterien der Objektivität und Reliabilität zu wahren, wurde die Analyse von zwei voneinander unabhängigen Promotionsstudentinnen durchgeführt (Intercoderreliabilität). Das Ergebnis des Prozesses ist in der Abbildung 5 zu sehen. 


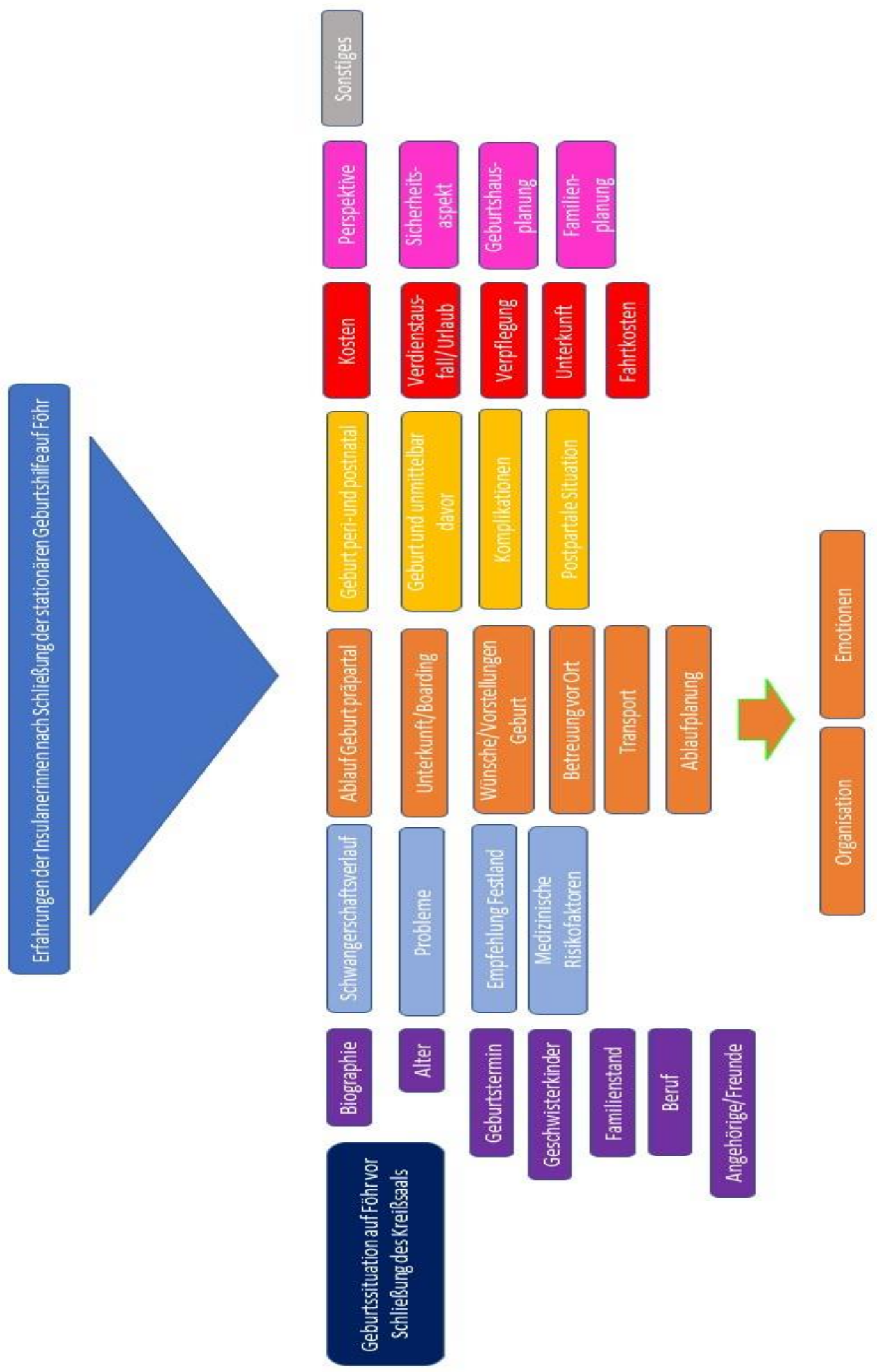




\subsubsection{Erstellen der Online-Umfrage und Einschlusskriterien}

Auf den qualitativ-empirischen Teil folgte die Ausarbeitung des quantitativ-empirischen Teils in Form einer Online-Umfrage. Die Erarbeitung des Online-Fragebogens fußte auf den Ergebnissen der qualitativen Inhaltsanalyse. Additiv wurden auch andere Fragebögen, die im Rahmen von Studien zu Geburtserfahrungen erstellt worden waren, gesichtet. Eine gute Orientierungshilfe bot ein Fragebogen zur Schwangerschaft, Geburt und zum poststationären Aufenthalt, der im Rahmen einer nationalen norwegischen Studie entwickelt worden war [58]. Eingeschlossen in die Online-Umfrage waren alle auf Föhr lebenden und gemeldeten Frauen, die ein Kind zwischen dem 01. Oktober 2015 und dem 30. September 2017 auf dem Festland geboren hatten. Es ging stets nur um das erste Kind der Teilnehmerin, das in diesem Zeitraum geboren wurde.

Die Online Umfrage wurde mit dem Open Source Programm „Lime Survey“, das von der ChristianAlbrechts-Universität zu Kiel zur Verfügung gestellt wurde, erstellt. Die Umfrage bestand aus sieben Frageblöcken mit insgesamt 64 Fragen (Online-Umfrage im Anhang II). Die Frageblöcke beinhalteten sowohl geschlossene Fragen, vor allem zur Biografie der Teilnehmerinnen und den Eckdaten zur Geburt des Kindes, als auch offene Fragen zur Erfahrung rund um die Geburt auf dem Festland. Um die persönlichen Einstellungen der Teilnehmerinnen zu erfassen, wurden zudem fünf-stufige Likertskalen verwendet

\subsubsection{Durchführung der Online-Umfrage}

Anfang Dezember 2017 wurden die Teilnehmerinnen postalisch zur Umfrage eingeladen. Dies erfolgte anhand eines offiziellen Anschreibens von Prof. Dr. med. Hanna Kaduszkiewicz vom Institut für Allgemeinmedizin der Universität zu Kiel, das an den Inselgeburt e.V. geschickt wurde. Der Verein hatte am 11. Dezember 2017 eine Vereinssitzung, in der die Anschreiben verteilt wurden. In dieser Sitzung waren Interessierte am Thema anwesend, darunter auch potenzielle Teilnehmerinnen sowie die Frauenärztin und zwei aktive Hebammen der Insel Föhr. Eine Hebamme sowie die Frauenärztin sicherten uns nach persönlichem Kontakt zu, die Anschreiben an alle potenziellen Teilnehmerinnen zu verteilen. Die Anschreiben wurden auch in der Frauenarztpraxis der Insel Föhr ausgelegt.

Die Teilnehmerinnen konnten wie folgt an der Umfrage teilnehmen. Interessierte Frauen mussten eine E-Mail an unsere Kontaktadresse schicken und bekamen daraufhin per E-Mail einen vollständig individualisierten und anonymisierten Zugangsschlüssel, mit dem sie an der Online-Umfrage teilnehmen konnten. Vor der Umfrage wurden die Teilnehmerinnen über die datenschutzrechtlichen Aspekte der Online-Umfrage sowie über ihre Einwilligung in die Nutzung der personenbezogenen Daten schriftlich aufgeklärt. 
Die Durchführung einer elektronischen Umfrage hatte für unsere Studie mehrere Vorteile. Zum einen nahmen wir an, dass das Teilnehmerinnenkollektiv sehr jung sei und somit gut über die web-basierte Umfrageplattform erreicht werden konnte, zum anderen konnten wir durch die Versendung von individualisierten Zugangsschlüsseln eine Doppelteilnahme an der Studie nahezu ausschließen und die Anonymität der Teilnehmerinnen zuverlässig sicherstellen. Vorteilhaft war auch, dass die Umfrage zeitlich und räumlich unabhängig ausgefüllt werden konnte. Ein weiterer Vorteil war, dass individualisiert je nach Antwort der Frauen automatische Filterfunktionen in den Fragebogen eingebaut werden konnten. Uns schien es auch sinnvoller, die Umfrage online in Umlauf zu bringen, da wir selbst nicht vor Ort sein konnten und keine postalischen Adressen der Teilnehmerinnen hatten. Weiterhin können durch das Durchführen einer elektronischen Umfrage keine Fehler durch manuelle Datenerfassung passieren und die Daten sind zur Auswertung sofort auf dem Server verfügbar $[59,60]$.

Der elektronische Fragebogen wurde am 10. Dezember 2017 freigeschaltet. Parallel zur Verteilung der Anschreiben durch die Hebamme und die Frauenärztin der Insel Föhr wurde über soziale Medien auf die Studie aufmerksam gemacht. Hier wurde vor allem die Homepage und die Facebook-Seite des Inselgeburt e.V. genutzt. Mitte Januar 2018 wurden alle Frauen, die an den Interviews teilgenommen und eingewilligt hatten bei Bedarf nochmal kontaktiert zu werden, nochmals telefonisch kontaktiert. Die Frauen wurden gebeten, weitere potenzielle Teilnehmerinnen über die Studie zu informieren. Des Weiteren erschien Ende Januar ein Artikel zur Dissertation im „Inselboten“, der lokalen Ausgabe der Schleswig Holsteiner Zeitung auf Föhr [61]. Um eine weitere Erhöhung der Rücklaufquote zu erreichen, wurden Mitte Februar 2018 nochmal alle Kontaktpersonen telefonisch angesprochen und ein weiterer Aufruf zur Teilnahme über Facebook geschaltet. Am 04. März 2018 wurde die Online-Umfrage beendet.

\subsubsection{Auswertung der Online-Umfrage}

Der Datensatz der Umfrage lag nach Abschluss der Umfrage in elektronischer Form vor. Die Bearbeitung des Datensatzes wurde zwischen den beiden Promotionsstudentinnen, die diese Studie betreuen, gemäß dem Schwerpunkt im Forschungsbereich aufgeteilt und die Ergebnisse wurden jeweils getrennt berechnet. Der Datensatz wurde zur weiteren statistischen Bearbeitung von „Lime Survey“ in Microsoft Excel 2016 exportiert. 


\subsection{Ethikvotum}

Das positive Votum der Ethikkommission der Medizinischen Fakultät der Christian-AlbrechtsUniversität zu Kiel zur Durchführung der Online-Befragung liegt vor (D564/17). Das Projekt wurde aus Eigenmitteln des Instituts für Allgemeinmedizin der Universität Kiel finanziert. Teilnehmerinnen der Studie erhielten keine Aufwandsentschädigung. Die in der Studie verwendeten Einverständniserklärungen zur Durchführung der Interviews liegen dem Institut für Allgemeinmedizin vor (siehe Anhang V). 


\section{Ergebnisse}

\subsection{Merkmale der Teilnehmerinnen der Online-Umfrage}

An der Online-Umfrage nahmen 29 Föhrer Frauen teil. In dem Beobachtungszeitraum vom 30. September 2015 bis zum 01. Oktober 2017 wurden auf der Insel Föhr laut des Amts Föhr/Amrum 111 Kinder geboren. Wenn man davon ausgeht, dass jeweils eine beantwortete Umfrage einem neugeborenen Kind entspricht, entspricht dies einer Rücklaufquote von 26,13 Prozent. Mehrlingsschwangerschaften finden dabei keine Berücksichtigung.

Das Teilnehmerinnenkollektiv gestaltete sich wie folgt:

24 Teilnehmerinnen waren gesetzlich versichert, fünft privat versichert. Das Durchschnittsalter bei Entbindung betrug 29,86 Jahre (SD \pm 4,45). Fünf Kinder wurden 2015 geboren, 132016 und zehn 2017. Ein Geburtstermin wurde von einer Teilnehmerin so angegeben, dass eine Zuordnung nicht möglich war, sodass hier nur eine Grundgesamtheit von 28 Teilnehmerinnen betrachtet werden kann. 20 Teilnehmerinnen haben in dem Beobachtungszeitraum ihr erstes Kind geboren. Für acht Teilnehmerinnen war die Geburt des Kindes in dem zu beobachtenden Zeitraum die zweite, für eine Teilnehmerin die dritte Geburt. 18 Teilnehmerinnen waren zum Zeitpunkt der Geburt verheiratet, 11 ledig, davon acht in einer festen Beziehung.

Tabelle 16: Charakteristika der Teilnehmerinnen an der Online-Umfrage

\begin{tabular}{|c|c|c|c|c|c|}
\hline & Anzahl & Prozent & & Anzahl & Prozent \\
\hline \begin{tabular}{|l} 
Versicherungsstatus $(n=29)$ \\
\end{tabular} & & & Beziehungsstatus ( $n=29$ ) & & \\
\hline Gesetzlich & 24 & 82,76 & Verheiratet & 18 & 62,07 \\
\hline Privat & 5 & 17,24 & In einer festen Beziehung & 11 & 37,93 \\
\hline Entbindungstermin $(n=28)$ & & & Das wievielte Kind ( $n=29$ ) & & \\
\hline 2015 & 5 & 17,86 & Das Erste & 20 & 68,97 \\
\hline 2016 & 13 & 46,43 & Das Zweite & 8 & 27,59 \\
\hline 2017 & 10 & 35,71 & Das Dritte & 1 & 3,45 \\
\hline \multicolumn{4}{|c|}{ Durchschnittsalter zum Zeitpunkt der Geburt } & \multicolumn{2}{|c|}{29,86 Jahre $\pm 4,45$ SD } \\
\hline
\end{tabular}




\subsection{Wege zum und vom Geburtsort}

18 Teilnehmerinnen besichtigten die Klinik im Vorfeld. Acht Teilnehmerinnen besichtigten auch die von ihnen gewählte Unterkunft im Vorfeld. Es wurde durchschnittlich eine Besichtigungsfahrt zur Klinik und/oder der Boarding-Einrichtung unternommen.

Zur Geburt selbst fuhren 23 Teilnehmerinnen mit dem Auto, eine wurde mit dem Hubschrauber ausgeflogen, zwei mit dem Seenotrettungskreuzer transportiert und im Anschluss mit dem Rettungswagen, zwei mit dem Rettungswagen.

Nach der Geburt wurden ausschließlich private PKWs benutzt, um auf die Insel Föhr zurück zu fahren.

Abbildung 6: Transportmittel zur Entbindung

\section{Transportmittel zur Entbindung}

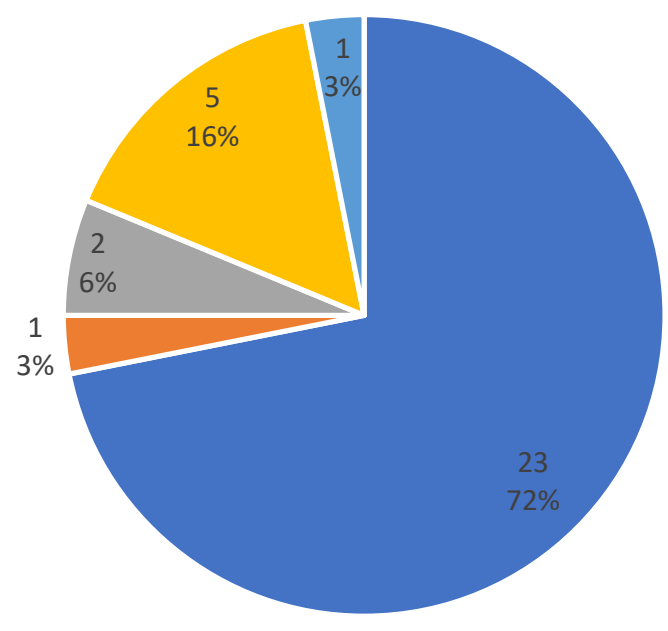

- Auto/Fähre - Hubschrauber \& Seenotrettungskreuzer \& Rettungswagen —Zu Fuß 


\subsection{Art, Ort der Unterkunft, Verweildauern auf dem Festland und Geburtsmodi}

14 Teilnehmerinnen haben das Diakonissenkrankenhaus Flensburg (Diako Flensburg) als Entbindungsort gewählt, elf Teilnehmerinnen wurden im Westküsten Klinikum (WKK) am Standort Heide entbunden, zwei Teilnehmerinnen in Bremen, eine im Klinikum Nordfriesland Standort Husum und eine Teilnehmerin in Offenburg.

\section{Abbildung 7: Ort der Entbindung}

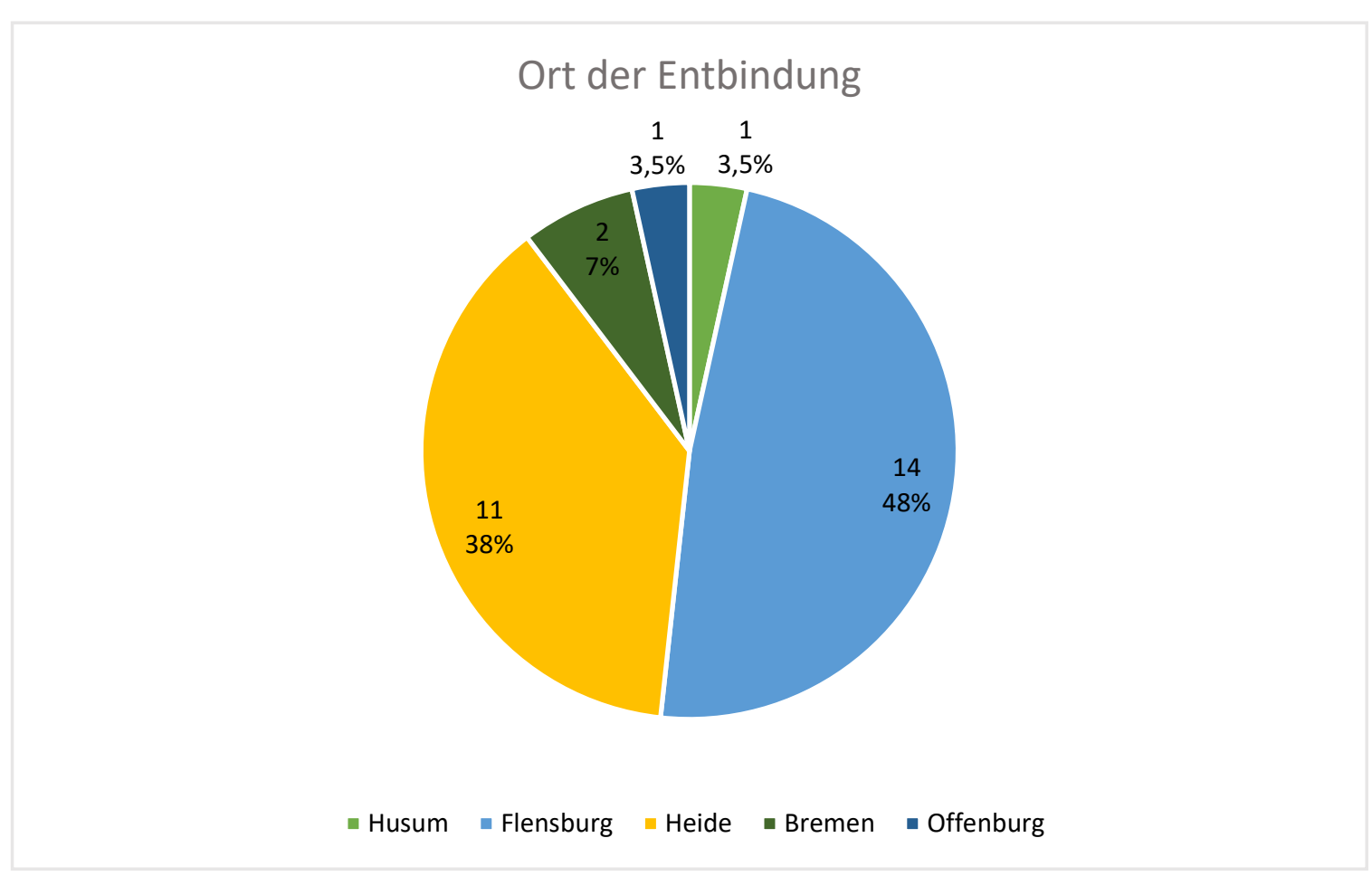

16 Teilnehmerinnen nahmen vor Ort eine Boarding-Einrichtung in Anspruch. Zu einer BoardingEinrichtung zählen alle Unterkünfte, die von der Entbindungsklinik im Vorfeld vermittelt werden, also auch Ferienwohnungen (FeWo). Unter der Annahme, dass die Nutzung des Hotels nicht im Vorfeld vermittelt wurde, sondern eher eine Notlösung darstellte, zählt das Hotel nicht zu einer BoardingEinrichtung. Acht Teilnehmerinnen wohnten bei Angehörigen der Familien oder Freund*innen auf dem Festland. Eine Teilnehmerin wohnte im Hotel, vier Teilnehmerinnen verbrachten die Zeit vor und nach der Geburt ausschließlich im Krankenhaus und nahmen daher kein Boarding in Anspruch. 


\section{Art der Unterkunft $(n=29)$}

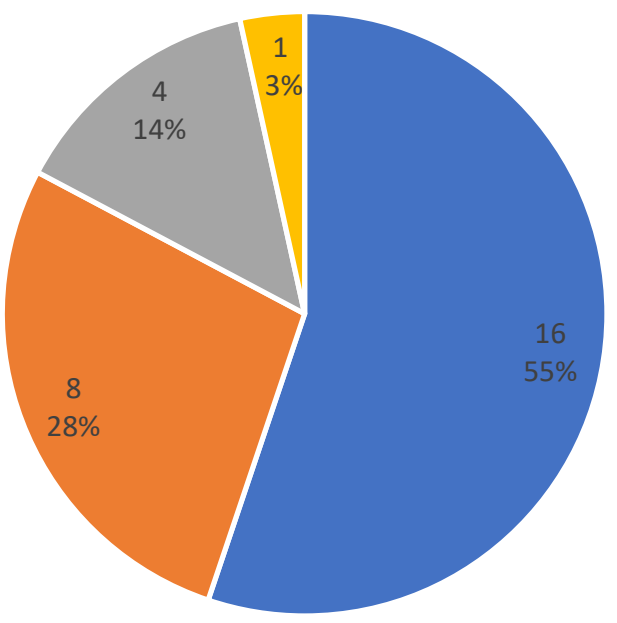

- Boarding/FeWo - Familie/Freunde - Krankenhaus = Hotel

Die durchschnittliche Aufenthaltsdauer auf dem Festland vor der Entbindung entsprach 9,55 Tage (IQR=13). Teilnehmerinnen, die in einer Boarding-Einrichtung unterkamen, verweilten prästationär kürzer auf dem Festland (im Durchschnitt acht Tage (IQR=12)) als Teilnehmerinnen, die bei der Familie wohnten (im Durchschnitt 17,625 Tage (IQR=11,25)). Frauen, die direkt ins Krankenhaus eingeliefert wurden, sowie die eine Teilnehmerin, die im Hotel wohnte, werden nicht in der unten dargestellten Grafik (Abbildung 9) dargestellt. 


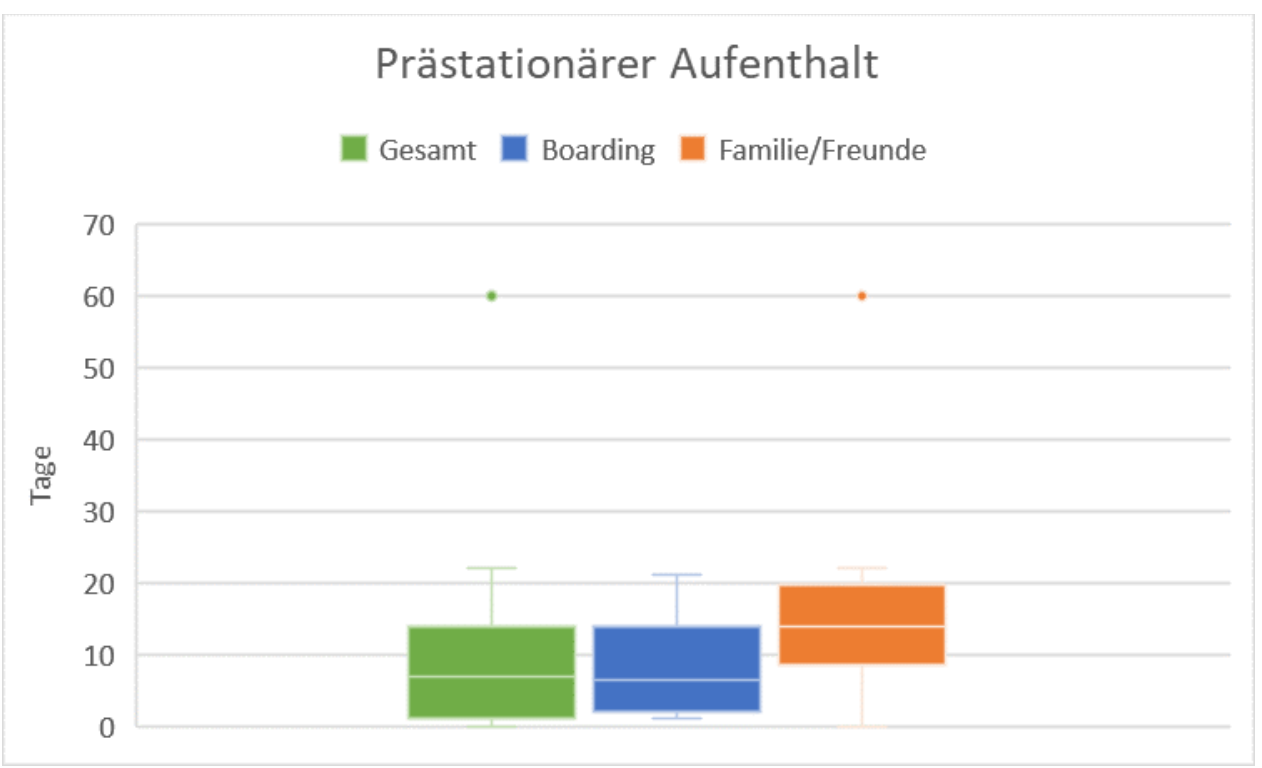

(Nicht dargestellt: Eine Teilnehmerin mit Hotelaufenthalt, sowie Frauen mit Krankenhausaufenthalt als einzigen Aufenthaltsort)

18 Teilnehmerinnen wurden spontan entbunden, bei sieben Teilnehmerinnen kam eine Saugglocke zum Einsatz und vier Teilnehmerinnen erhielten einen Kaiserschnitt. Aus dem Datensatz ist nicht ersichtlich, ob es sich um eine primäre oder sekundäre Sectio handelte.

Abbildung 10: Geburtsmodi der Teilnehmerinnen

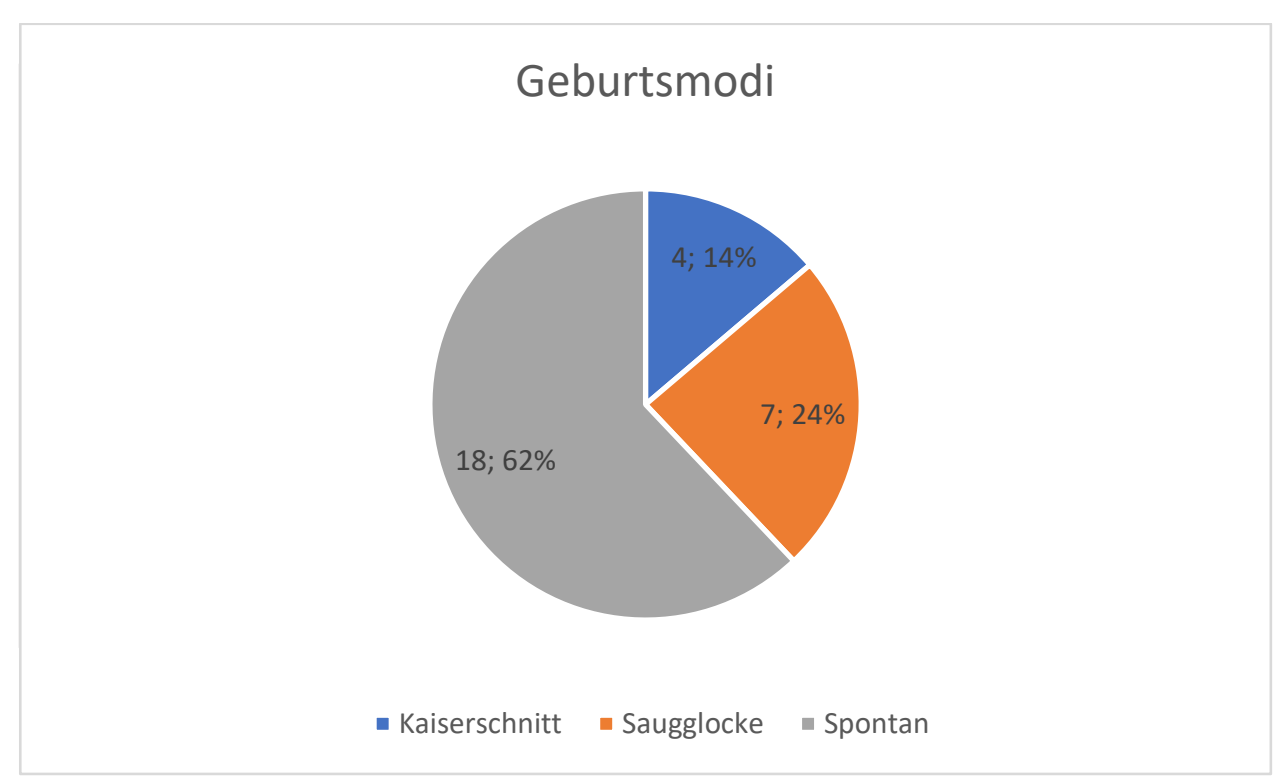


Die durchschnittliche Verweildauer in der Klinik lag bei 4,62 Tagen (IQR=2). Teilnehmerinnen, die spontan entbunden wurden, lagen im Durchschnitt 4,67 Tage (IQR=2) in der Klinik. Wurde mit einer Saugglocke entbunden, so lag die mittlere Verweildauer bei 4,71 Tagen (IQR=2). Frauen, die einen Kaiserschnitt bekamen, lagen im Durchschnitt 4,25 Tage $(\mathrm{IQR}=1,75)$ in der Entbindungsklinik (Abbildung 10).

Abbildung 11: Stationäre Verweildauern der Teilnehmerinnen

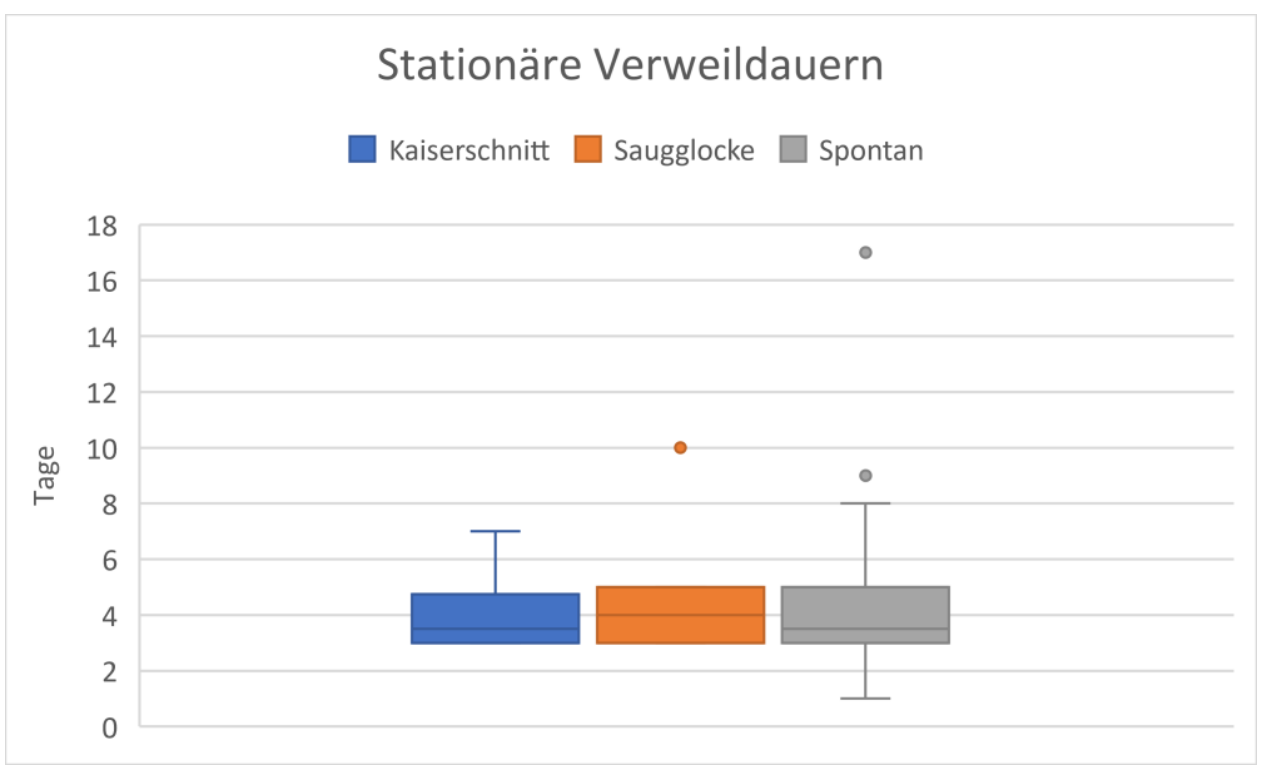




\subsection{Aufstellung der Kosten im Rahmen der Online-Umfrage}

\subsubsection{Direkte nicht-medizinische Kosten}

\subsubsection{Direkte nicht-medizinische Kosten der Teilnehmerinnen}

Die direkten nicht-medizinischen Kosten der Teilnehmerinnen wurden in der Umfrage unter den Kategorien „Verpflegungskosten“, „Fahrtkosten“, „Fähr- und Parkkosten“, , Unterkunftskosten“, sowie der Kategorie "andere Kosten“ abgefragt. Zudem wurden die Frauen gebeten nur die Kosten anzugeben, die nicht im Nachhinein von der Versicherung übernommen worden sind. Im Mittel betrugen die direkten nicht-medizinischen Kosten der Teilnehmerinnen 632 Euro (IQR=455).

Abbildung 12: Direkte nicht-medizinische Kosten der Teilnehmerinnen

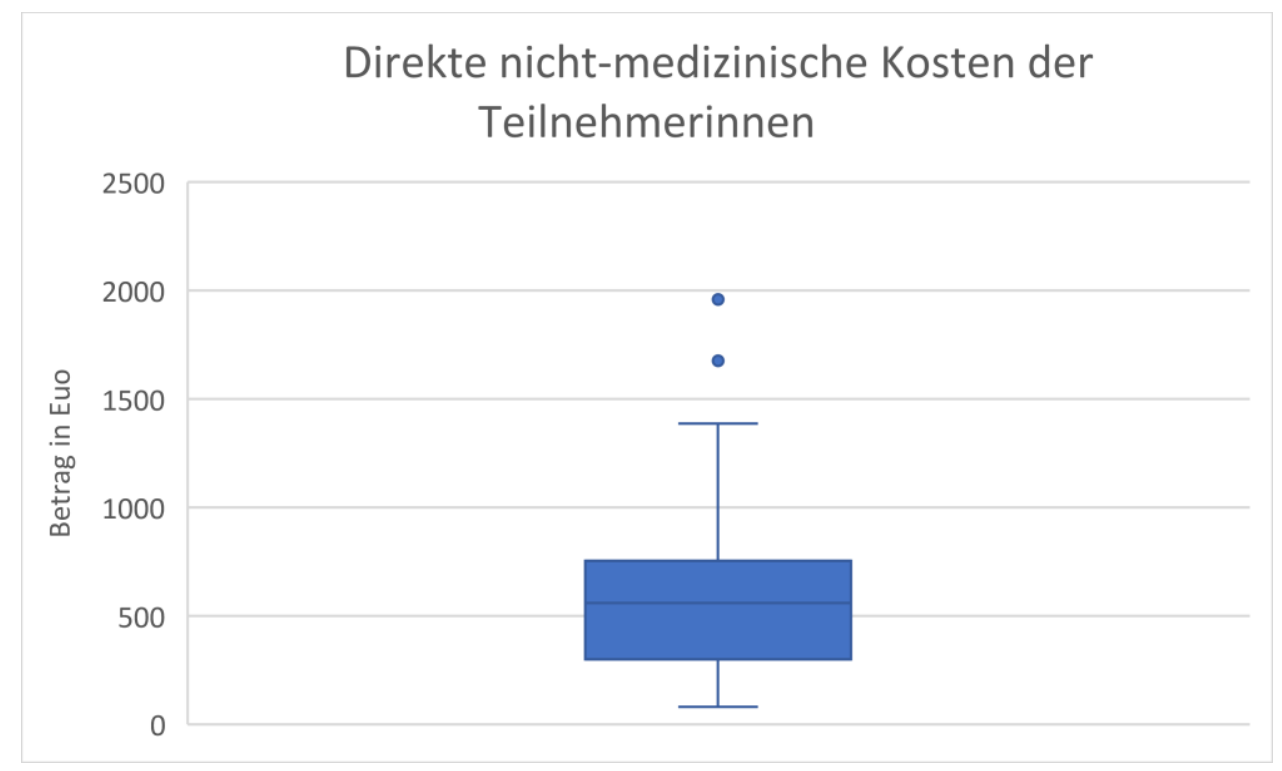


Tabelle 17: Direkte nicht- medizinische Kosten der Teilnehmerinnen in Euro

\begin{tabular}{lrrrrrrr}
\hline & Minimum & Maximum & 1. Quartil & Median & 3.Quartil & Mittelwert & SD \\
\hline Gesamt & 82 & 1.960 & 300 & 560 & 755 & 631,79 & 452,26 \\
\hline Verpflegung & 0 & 500 & 45 & 100 & 200 & 155,89 & 152,08 \\
\hline Fahrtkosten & 10 & 500 & 60 & 100 & 200 & 142,41 & 118,92 \\
\hline Fähr- und & 0 & 280 & 75 & 110 & 150 & 118,72 & 64,70 \\
Parkkosten & & & & & & & \\
\hline Unterkunft & 0 & 1.440 & 0 & 65 & 200 & 161,29 & 288,49 \\
\hline Sonstige & 0 & 400 & 0 & 0 & 200 & 75,18 & 112,54 \\
Kosten & & & & & & & \\
\hline
\end{tabular}

Da sich die Ausgaben für die verschiedenen Kostenkategorien in Abhängigkeit der Unterkunftsart unterscheiden, werden in den folgenden Boxplot-Grafiken noch einmal die Kostenkategorien anhand der gewählten Unterkunftsart aufgeschlüsselt (Abbildungen 12-16).

Abbildung 13: Verpflegungskosten der Teilnehmerinnen aufgeschlüsselt nach Unterkunftsart ( $n=29)$

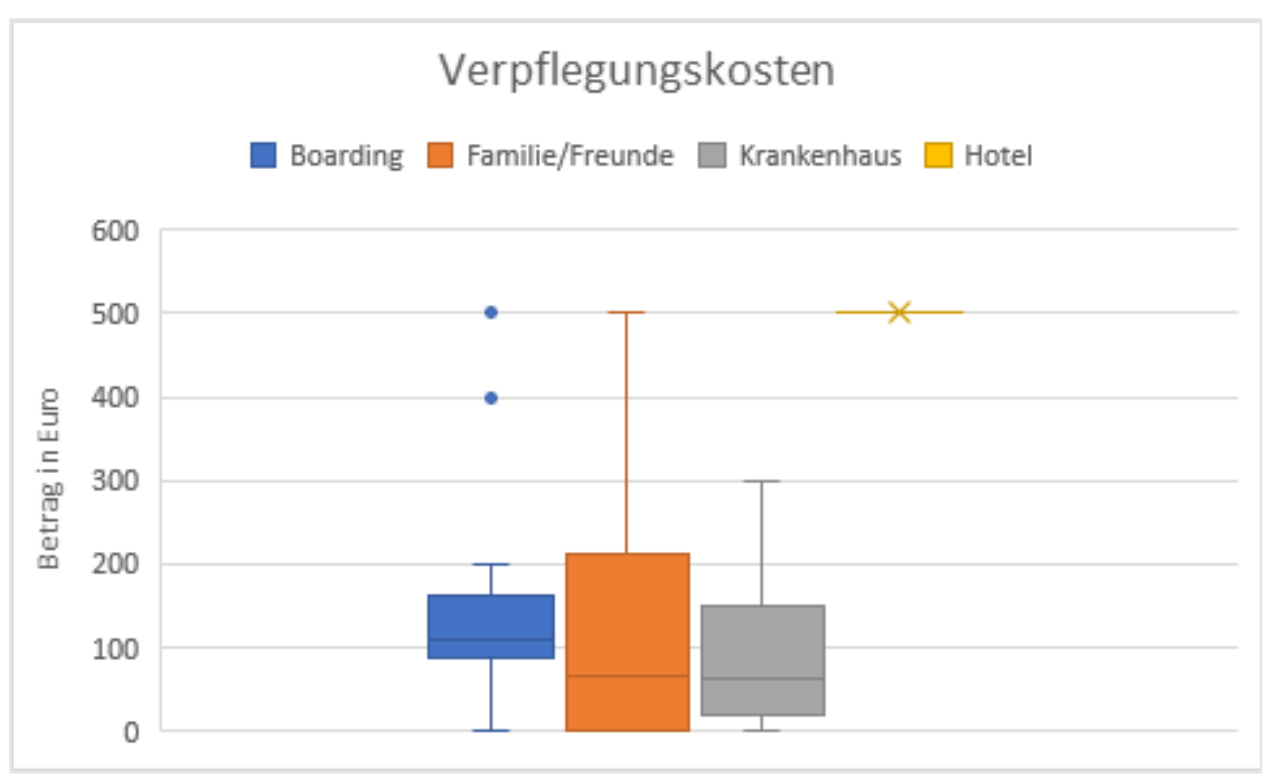


Abbildung 14: Fahrtkosten der Teilnehmerinnen aufgeschlüsselt nach Unterkunftsart ( $n=29)$

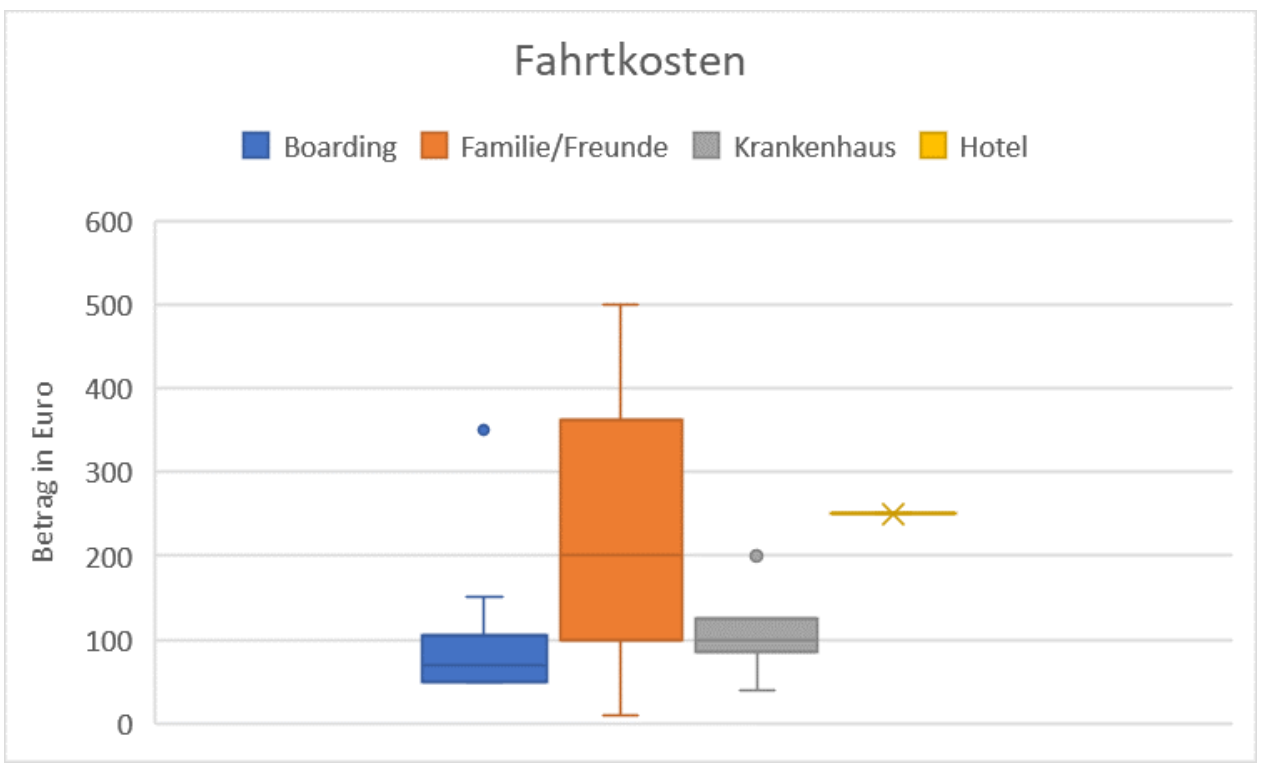

Abbildung 15: Fähr- und Parkkosten der Teilnehmerinnen aufgeschlüsselt nach Unterkunftsart ( $n=29)$

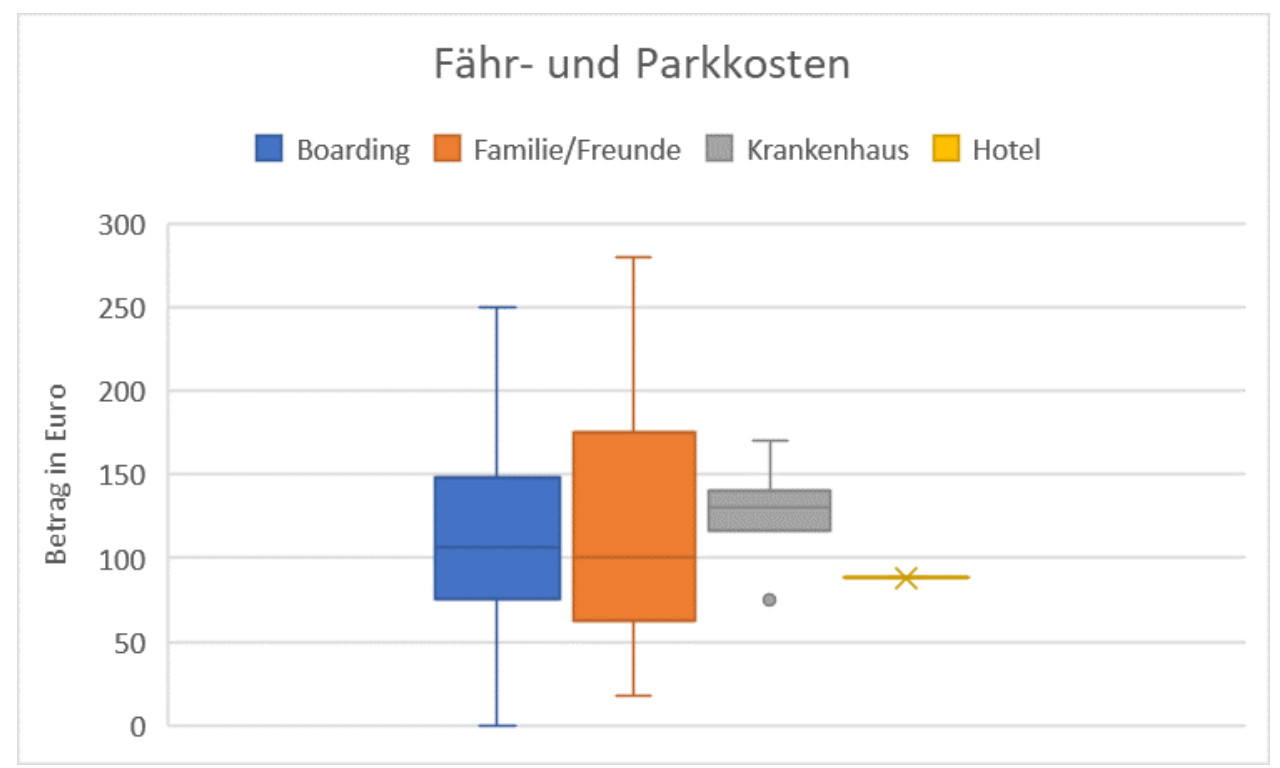


Abbildung 16: Unterkunftskosten der Teilnehmerinnen aufgeschlüsselt nach Unterkunftsart $(n=28)^{*}$

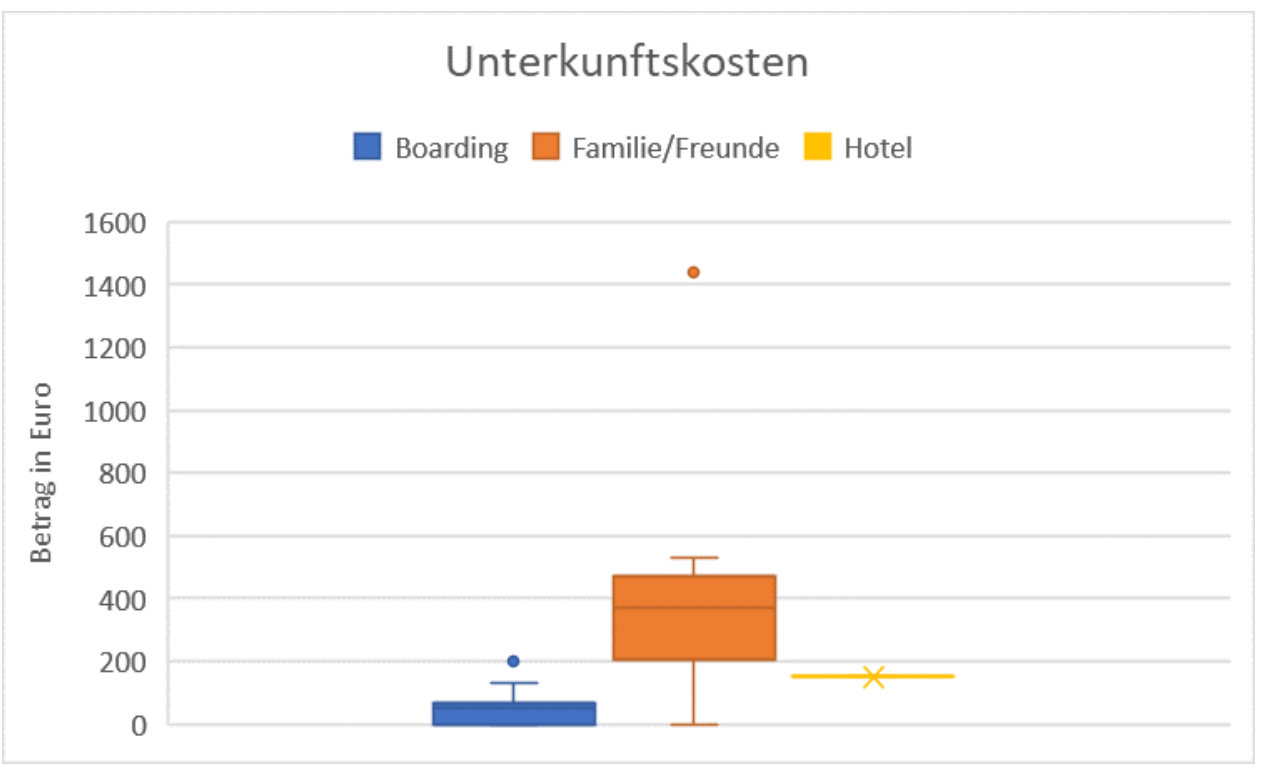

*Da bei einem direkten Krankenhausaufenthalt den Teilnehmerinnen keine Kosten bezüglich der Unterkunft entstanden, wurde diese Unterkunftsart nicht in Abbildung 16 aufgenommen.

Bei den Frauen, die das Boarding-Angebot in Anspruch nahmen, entstanden die zusätzlichen Kosten hauptsächlich dadurch, dass der Partner nach der Entbindung die Boarding-Unterkunft selbst zahlen musste und nur bis zur Entbindung kostenlos mit der Partnerin in der Boarding-Einrichtung wohnen durfte (Transkript 1; Absatz 141). Für die Unterkunftskosten bei Familien und Freund*innen wurde eine Übernachtungspauschale von 24 Euro pro Kalendertag gemäß §9 des Einkommenssteuergesetzes angenommen. 
Abbildung 17: Sonstige Kosten der Teilnehmerinnen aufgeschlüsselt nach Unterkunftsart ( $n=29)$

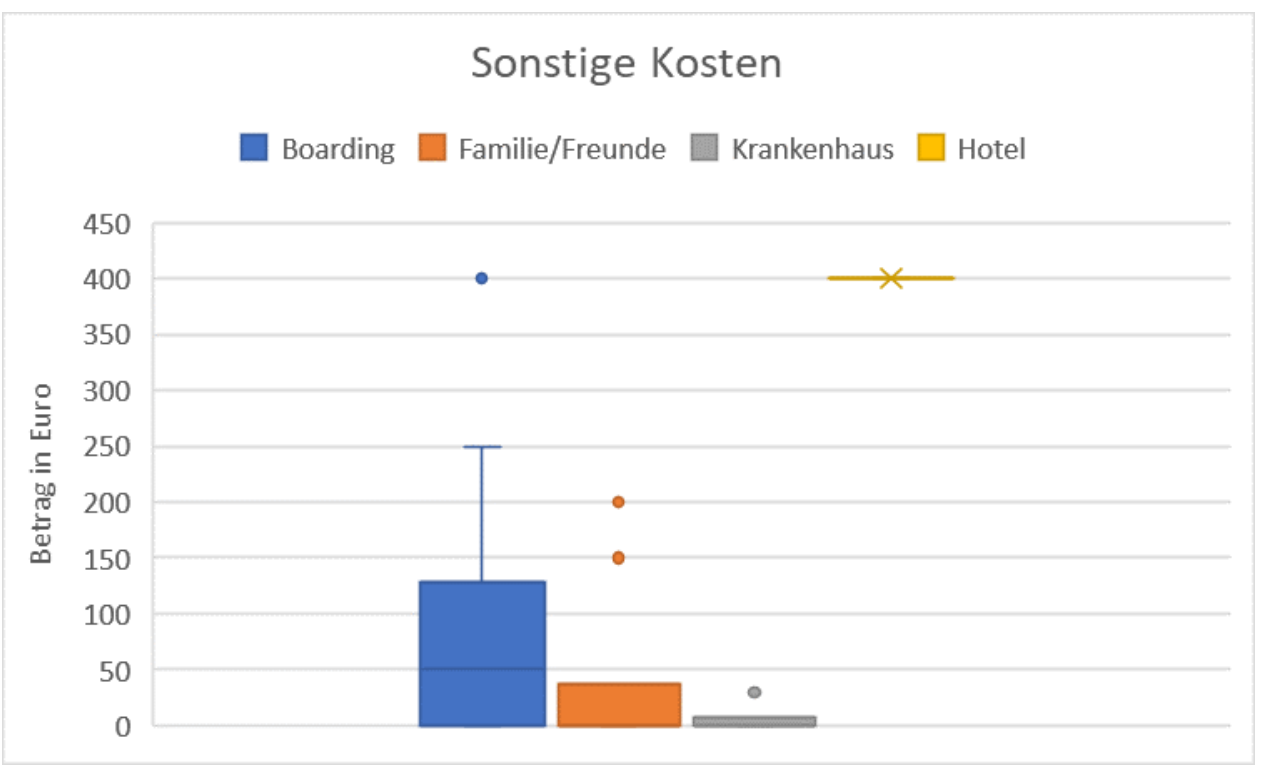

Die sonstigen Kosten umfassten Kosten, die zur Freizeitgestaltung anfielen, wie zum Beispiel Besuche im Freizeitpark, im Schwimmbad, in Museen oder im Kino. Hierbei ist anzumerken, dass bei neun Frauen der Stichprobe auch Geschwisterkinder mit auf dem Festland waren und dementsprechend mitversorgt werden mussten oder für deren Betreuung durch Angehörige gesorgt werden musste. Allerdings lassen sich in diesen Fällen nur schwer konkrete Kosten zuordnen, so dass dieser Aspekt nur schlecht in den „sonstigen Kosten“ repräsentiert ist. 
Tabelle 18: Zusammenfassung der direkten nicht-medizinischen Kosten in Abhängigkeit der gewählten Unterkunft in Euro $(n=29)$

\section{Minimum Maximum $\quad$ 1. Median 3.Quartil Mittelwert SD Quartil}

\begin{tabular}{|c|c|c|c|c|c|c|c|}
\hline Verpflegung & & & & & & & \\
\hline Boarding & 0 & 500 & 87,5 & 110 & 162,5 & 147,5 & 133,14 \\
\hline Familie/Freunde & 0 & 500 & 0 & 65 & 212,5 & 135 & 176,31 \\
\hline Krankenhaus & 0 & 300 & 18,75 & 62,5 & 150 & 106,25 & 135,98 \\
\hline Hotel & - & - & - & - & - & 500 & - \\
\hline Fahrtkosten & & & & & & & \\
\hline Boarding & 50 & 350 & 50 & 70 & 105 & 98,75 & 75,53 \\
\hline Familie/Freunde & 10 & 500 & 100 & 200 & 362,5 & 232,5 & 169,09 \\
\hline Krankenhaus & 40 & 200 & 85 & 100 & 125 & 110 & 66,33 \\
\hline Hotel & - & - & - & - & - & 250 & - \\
\hline
\end{tabular}

Fähr- und

Parkkosten

\begin{tabular}{|c|c|c|c|c|c|c|c|}
\hline Boarding & 0 & 250 & 75 & 106,5 & 147,75 & 113,13 & 63,45 \\
\hline Familie/Freunde & 18 & 280 & 62,25 & 100 & 175 & 124,63 & 89,77 \\
\hline Krankenhaus & 75 & 170 & 116,25 & 130 & 140 & 126,25 & 39,02 \\
\hline Hotel & - & - & - & - & - & 88 & - \\
\hline \multicolumn{8}{|c|}{ Unterkunftskosten } \\
\hline Boarding & 0 & 200 & 0 & 50 & 70 & 56,88 & 68,29 \\
\hline Familie/Freunde & 0 & 1440 & 204 & 372 & 474 & 432 & 450,65 \\
\hline Hotel & - & - & - & - & - & 150 & - \\
\hline \multicolumn{8}{|l|}{ Sonstige Kosten } \\
\hline Boarding & 0 & 400 & 0 & 50 & 127,5 & 87,5 & 111,15 \\
\hline Familie/Freunde & 0 & 200 & 0 & 0 & 37,5 & 43,75 & 82,1 \\
\hline Krankenhaus & 0 & 30 & 0 & 0 & 7,5 & 7,5 & 15 \\
\hline Hotel & - & - & - & - & - & 400 & - \\
\hline
\end{tabular}

\subsubsection{Direkte nicht-medizinische Kosten der Leistungsträger nach Schließung der Geburtshilfestation auf Föhr}

Um nach der Schließung der Geburtshilfestation auf Föhr eine Gesamtkostenrechnung der direkten nicht-medizinischen Kosten zu erstellen, wird in dieser Dissertation davon ausgegangen, dass pro Jahr 
eine Luftrettung, eine Seenotrettung mit konsekutiver Rettungsfahrt zur Entbindungsklinik und eine Rettung im Rettungswagen jeweils in das Diakonissenkrankenhaus nach Flensburg durchgeführt werden. Diese Annahmen sind realistisch, da die Daten aus unserer Umfrage belegen, dass innerhalb des Umfragezeitraums von zwei Jahren fünf Teilnehmerinnen durch den Rettungsdienst zur Entbindung auf das Festland gebracht werden mussten. Eine Teilnehmerin wurde mit dem Rettungswagen zum Flugplatz Wyk auf Föhr gebracht (als Distanz werden zehn Kilometer angenommen) und dann mit dem Rettungshubschrauber zur Geburt ausgeflogen. Zwei Teilnehmerinnen wurden mit dem Seenotrettungskreuzer von der Insel und anschließend mit einem Rettungswagen in das Zielkrankenhaus gebracht. Zwei weitere wurden ausschließlich mit dem Rettungswagen zum Ort der Entbindung auf dem Festland transportiert.

Des Weiteren fallen für die Versicherungen als direkte nicht-medizinische Kosten die Kosten für das Boarding und eine Pauschale für den Hebammennotruf an. Aus der Umfrage geht hervor, dass die Teilnehmerinnen im Schnitt acht Tage prästationär in einer Boarding-Einrichtung gewohnt hatten. Im Mittel fanden auf Föhr von 2010 bis 2014 jährlich 56 Geburten statt. Wenn wir diese Geburtenzahl nun als Richtwert für die Jahre nach der Schließung annehmen, ergeben sich 448 Boardingtage pro Jahr, die die Krankenkassen übernehmen. Hinzu kommt der Zuschuss der Krankenkassen zum Hebammennotruf in Höhe von 9.600 Euro pro Jahr.

Als weiterer Leistungsträger tritt der Kreis Nordfriesland auf. $\mathrm{Er}$ übernimmt die Haftpflichtversicherungen von zwei Hebammen auf Föhr und zahlt 45 Euro pro Tag für den Hebammennotruf. Zusätzlich übernimmt der Kreis Nordfriesland die Förderung des Rufdienstes der durchführenden Fachärztin für Frauenheilkunde und Geburtshilfe sowie einen Anteil von 5820,75 Euro an der Haftpflichtversicherung für den Zeitraum vom 01. April 2017 bis zum 31.12.2017 (275 Ruftage) [53]. Zwischen der Schließung der Geburtshilfe zum 01. Oktober 2015 und dem 31.03.2017 (90 Ruftage im Jahr 2017) wurde der Rufdienst weiterhin vom Klinikum Nordfriesland finanziert.

Daraus ergeben sich, gemäß in der Tabelle 15 „Verbrauch und monetäre Bewertungen der Ressourcen" in Kapitel 2 aufgeführten Ressourcenbewertungen folgende direkte nicht-medizinische Kosten der Leistungsträger nach Schließung der Geburtshilfe auf Föhr. Die zuletzt genannten Zahlen beziehen sich ausschließlich auf die Situation nach der Schließung der Geburtshilfe im Jahr 2017. 
Tabelle 19: Gesamte direkte nicht-medizinische Kosten der Leistungsträger nach Schließung der Geburtshilfe auf Föhr (exemplarisch Jahr 2017)

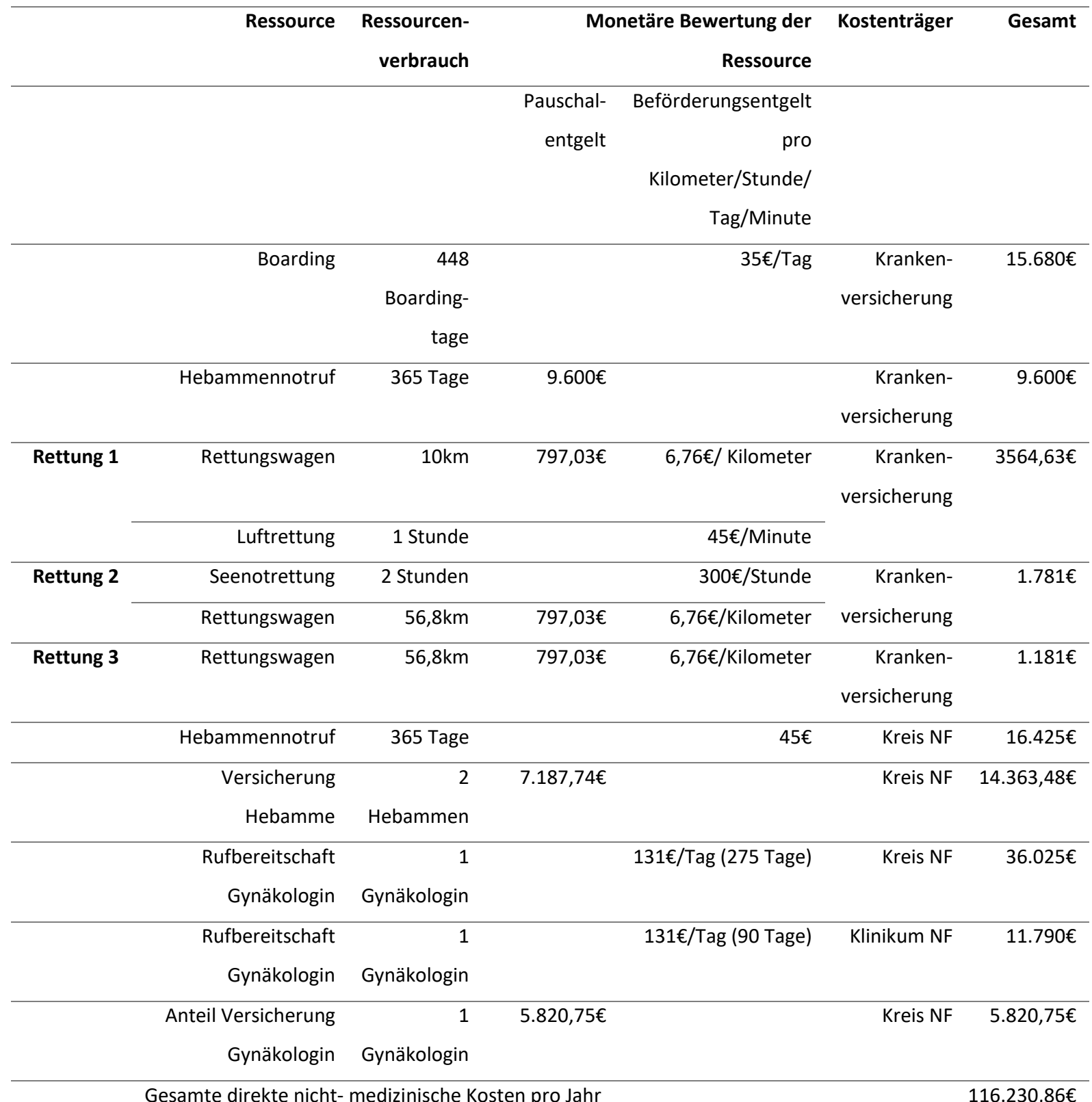

\subsubsection{Direkte nicht- medizinische Kosten der Leistungsträger vor Schließung der Geburtshilfestation auf Föhr}

Um einen Vorher-Nachher-Vergleich zu erstellen und mögliche inkrementelle Kosten oder Kostenverschiebungen zu erkennen ist es nötig, soweit möglich, auch die direkten nicht-medizinischen Kosten der Leistungsträger vor Schließung der Geburtshilfe auf Föhr zu berücksichtigen. Die Erfassung dieser Kosten gestaltete sich als höchst schwierig, da nach eingehender Recherche widersprüchliche Angaben vom Klinikum Nordfriesland, dem Kreis Nordfriesland und Angaben einer Hebamme bestehen. Daher geben diese Zahlen nur einen sehr groben Richtwert an. 
Der Kreis Nordfriesland gab auf Nachfrage an, dass vor Schließung der Geburtshilfe auf Föhr die zwei Hebammen, die Geburtshilfe auf Föhr leisteten, je einen Betrag von 355Euro pro Monat für die Sicherstellung der Rufbereitschaft auf Föhr vom Klinikum Nordfriesland erhalten haben, sowie ein Anteil an der Haftpflichtversicherung der Hebammen übernommen wurde [62]. Diese Angabe konnte aber nach einem Telefonat mit dem Geschäftsführer des Klinikums Nordfriesland nicht bestätigt werden. Auch eine befragte Hebamme verneinte einen Zuschuss zur Haftpflichtversicherung durch das Klinikum Nordfriesland. Daher wird der Anteil an der Haftpflichtversicherung nicht als Kostenparameter in der nachstehenden Tabelle berücksichtigt.

Allerdings gab der Geschäftsführer des Klinikums an [63], dass das Klinikum Nordfriesland die Haftpflichtversicherung der Gynäkologin bis zu einem Maximalbetrag von 20.000 Euro pro Jahr mitfinanzierte und der Gynäkologin eine Einsatzpauschale von 131 Euro pro 24-Stunden-Rufdienst vor Schließung der Geburtshilfestation auf Föhr zahlte. Es wird davon ausgegangen, dass die Rufbereitschaft 365 Tage im Jahr bestand und im Falle einer Abwesenheit der Gynäkologin durch eine/n Vertretungsarzt/Vertretungsärztin übernommen wurde.

Tabelle 20: Gesamte direkte nicht- medizinische Kosten pro Jahr vor Schließung der Geburtshilfe auf Föhr

\begin{tabular}{|c|c|c|c|c|c|}
\hline \multirow[t]{3}{*}{ Ressource } & \multirow{3}{*}{$\begin{array}{l}\text { Ressourcen- } \\
\text { verbrauch }\end{array}$} & \multicolumn{2}{|c|}{ Monetäre Bewertung der Ressource } & \multirow[t]{3}{*}{ Kostenträger } & \multirow[t]{3}{*}{ Gesamt } \\
\hline & & Pauschal- & Beförderungsentgelt pro & & \\
\hline & & entgelt & Kilometer/Stunde/Tag/Minute & & \\
\hline \multirow[t]{2}{*}{ Hebammennotruf } & 2 & & $355 € /$ Monat & Klinikum NF & $8.520 €$ \\
\hline & Hebammen & & & & \\
\hline Rufbereitschaft & 1 & & $131 € /$ Tag & Klinikum NF & $47.815 €$ \\
\hline Gynäkologin & Gynäkologin & & & & \\
\hline Haftpflichtversicherung & 1 & $20.000 €$ & & Klinikum NF & $20.000 €$ \\
\hline Gynäkologin & Gynäkologin & & & & \\
\hline & Gesam & e direkte nic & ht medizinische Kosten pro Jahr & & $76.335 €$ \\
\hline
\end{tabular}

\subsubsection{Direkte medizinische Kosten}

Multipliziert man nun die Kosten für eine einzelne Geburt in einer belegärztlich geführten Geburtshilfe (siehe Kapitel 2.4.2) mit den Fallzahlen der Inselklinik Föhr/Amrum für den Zeitraum 2010 bis 2015, resultieren hieraus die direkten jährlichen medizinischen Kosten der stationären Geburtshilfe auf Föhr aus der Sicht der Krankenkassen. Da für das Jahr 2010 keine genauen Angaben zu Geburtsmodi vorhanden sind, wird von der deutschlandweiten Sectiorate von 31,9 Prozent im Jahr 2010 [1] ausgegangen und es werden nur primäre Sectiones betrachtet. Das Jahr 2015 kann nur bedingt 
betrachtet werden, da die Schließung der Geburtshilfe auf Föhr bereits am 30. September 2015 erfolgte.

Tabelle 21: Direkte medizinischen Kosten in Euro der stationären Geburtshilfe auf Föhr

\begin{tabular}{|c|c|c|c|c|c|c|c|c|c|c|c|c|}
\hline & \multicolumn{2}{|c|}{2010} & \multicolumn{2}{|c|}{2011} & \multicolumn{2}{|c|}{2012} & \multicolumn{2}{|c|}{2013} & \multicolumn{2}{|c|}{2014} & \multicolumn{2}{|c|}{2015} \\
\hline $\begin{array}{l}\text { Spontan- } \\
\text { geburt }\end{array}$ & $\begin{array}{l}4 \\
4\end{array}$ & $\begin{array}{l}61.895,6 \\
8\end{array}$ & 35 & $51.477,3$ & 33 & $49.275,6$ & 35 & $52.663,8$ & 44 & $\begin{array}{l}68.231,2 \\
4\end{array}$ & 23 & $\begin{array}{l}36.361,8 \\
5\end{array}$ \\
\hline $\begin{array}{l}\text { Primäre } \\
\text { Sectio }\end{array}$ & $\begin{array}{l}2 \\
0\end{array}$ & $46.348,4$ & 6 & $\begin{array}{l}14.081,2 \\
8\end{array}$ & 8 & $\begin{array}{l}18.923,4 \\
4\end{array}$ & 8 & $\begin{array}{l}19.167,3 \\
6\end{array}$ & 10 & $24.934,7$ & 13 & $\begin{array}{l}33.420,1 \\
4\end{array}$ \\
\hline $\begin{array}{l}\text { Sekundäre } \\
\text { Sectio }\end{array}$ & & & 8 & $\begin{array}{l}21.567,5 \\
2\end{array}$ & 6 & $\begin{array}{l}16.226,9 \\
4\end{array}$ & 15 & $\begin{array}{l}41.224,9 \\
5\end{array}$ & 10 & $28.394,9$ & 9 & $\begin{array}{l}26.066 .2 \\
5\end{array}$ \\
\hline Gesamt & & $8.244,08$ & 87. & 26,1 & 84. & 25,98 & & 056,11 & 121 & 560,84 & 95. & 48,24 \\
\hline
\end{tabular}

Im Durchschnitt betragen die direkten medizinischen Kosten der stationären Geburtshilfe auf Föhr für spontane Geburten sowie primäre und sekundäre Sectiones pro Jahr demnach 101.710,23 Euro ( \pm SD $13609,2614)$.

Für die direkten medizinischen Kosten nach der Schließung der stationären Geburtshilfe auf Föhr nehmen wir wieder eine Geburtenzahl von 56 pro Jahr an. Aus den Daten der Umfrage geht hervor, dass Flensburg als Entbindungsort von 48 Prozent der Teilnehmerinnen bevorzugt gewählt wurde. 38 Prozent der Teilnehmerinnen entschieden sich für das WKK Heide. Die restlichen Teilnehmerinnen wählten überwiegend aufgrund familiärer Begebenheiten weiter weg gelegene Krankenhäuser. Wir nehmen daher an, dass von den oben genannten 56 Geburten pro Jahr 26 Geburten in dem Diakonissenkrankenhaus Flensburg, einer gynäkologischen Hauptabteilung, stattfinden. 21 Geburten finden demnach im WKK Heide statt, das eine gynäkologische Abteilung mit Beleghebammen besitzt. Für die restlichen neun Geburten wurde angenommen, dass sie in einer gynäkologischen Hauptabteilung ohne Beleghebammen stattfinden. Daher werden sie dem Diakonissenkrankenhaus Flensburg zugerechnet.

Wir betrachten die Jahre 2010 bis 2016 mit einer jährlichen Geburtenrate von 56. Berücksichtigt werden nur Spontangeburten und primäre Sectiones. Auch hier wird eine Sectiorate von 31,9 Prozent angenommen. Aus den Tabellen 2.4.3. und 2.4.4. lassen sich somit die direkten medizinischen Kosten für eine Geburt in Flensburg und Heide berechnen. 
Tabelle 22: Direkte medizinische Kosten in Euro bei Entbindung auf dem Festland

\begin{tabular}{|l|r|r|r|r|r|r|r|r|r|}
\hline & \multicolumn{2}{|l|}{} & $\mathbf{2 0 1 0}$ & $\mathbf{2 0 1 1}$ & $\mathbf{2 0 1 2}$ & $\mathbf{2 0 1 3}$ & $\mathbf{2 0 1 4}$ & $\mathbf{2 0 1 5}$ & $\mathbf{2 0 1 6}$ \\
\hline $\begin{array}{l}\text { Diako } \\
\text { Flensburg }\end{array}$ & Spontangeburt & 24 & $34.745,52$ & $35.310,72$ & $37.964,64$ & $39.036,00$ & $40.775,04$ & $43.344,00$ & $44.531,04$ \\
\cline { 2 - 9 } & Primäre Sectio & 11 & $25.222,56$ & $25.735,82$ & $27.056,59$ & $27.268,01$ & $29.010,19$ & $29.788,00$ & $30.290,48$ \\
\hline \multirow{2}{*}{ WKK } & Spontangeburt & 15 & $22.128,60$ & $22.353,90$ & $23.702,70$ & $24.044,85$ & $25.056,3$ & $25.971,75$ & $26.560,35$ \\
\cline { 2 - 9 } & Primäre Sectio & 6 & $14.882,22$ & $15.051,48$ & $15.684,78$ & $15.762,54$ & $16.568,94$ & $16.840,56$ & $17.134,62$ \\
\hline & Gesamt & 56 & $96.978,9$ & $98.451,92$ & $104.408,71$ & $106.111,4$ & $111.410,47$ & $115.944,31$ & $118.516,49$ \\
\hline
\end{tabular}

Diese betragen durchschnittlich unter der Annahme der Präferenzen der Teilnehmerinnen bezüglich des Entbindungsortes aus der Umfrage pro Jahr insgesamt 107.403,17 Euro ( \pm SD 7671,3)

\subsubsection{Indirekte Kosten}

In der Umfrage befragten wir die Teilnehmerinnen, ob sie oder ihr Partner einen Verdienstausfall durch die Geburt auf dem Festland hatten. Zwei Teilnehmerinnen gaben an, selbst einen Verdienstausfall gehabt zu haben, da sie selbstständig arbeiteten. Weitere sechs gaben an, dass zumindest ihr Partner aufgrund von Selbstständigkeit einen Verdienstausfall erlitten hat, in dem zum Beispiel der Betrieb schließen musste, damit der Partner die Teilnehmerin auf das Festland begleiten konnte. Insgesamt gaben demnach acht Teilnehmerinnen indirekte Kosten an, das entspricht 27,6 Prozent der Stichprobe. Die folgende Boxplot-Grafik bezieht sich nur auf die Frauen, die indirekte Kosten angegeben haben $(n=8)$.

Tabelle 23: Indirekte Kosten der Teilnehmerinnen in Euro ( $n=8)$

\begin{tabular}{llllllll}
\hline & Minimum & Maximum & 1. Quartil & Median & 3. Quartil & Mittelwert & SD \\
\hline Indirekte & 120 & 3.000 & 615 & 1.200 & $2.187,5$ & $1.416,25$ & $1.001,9$ \\
Kosten $(\mathbf{n}=\mathbf{8})$ & & & & & & & \\
\hline
\end{tabular}


Abbildung 18: Indirekte Kosten der Teilnehmerinnen ( $n=8)$

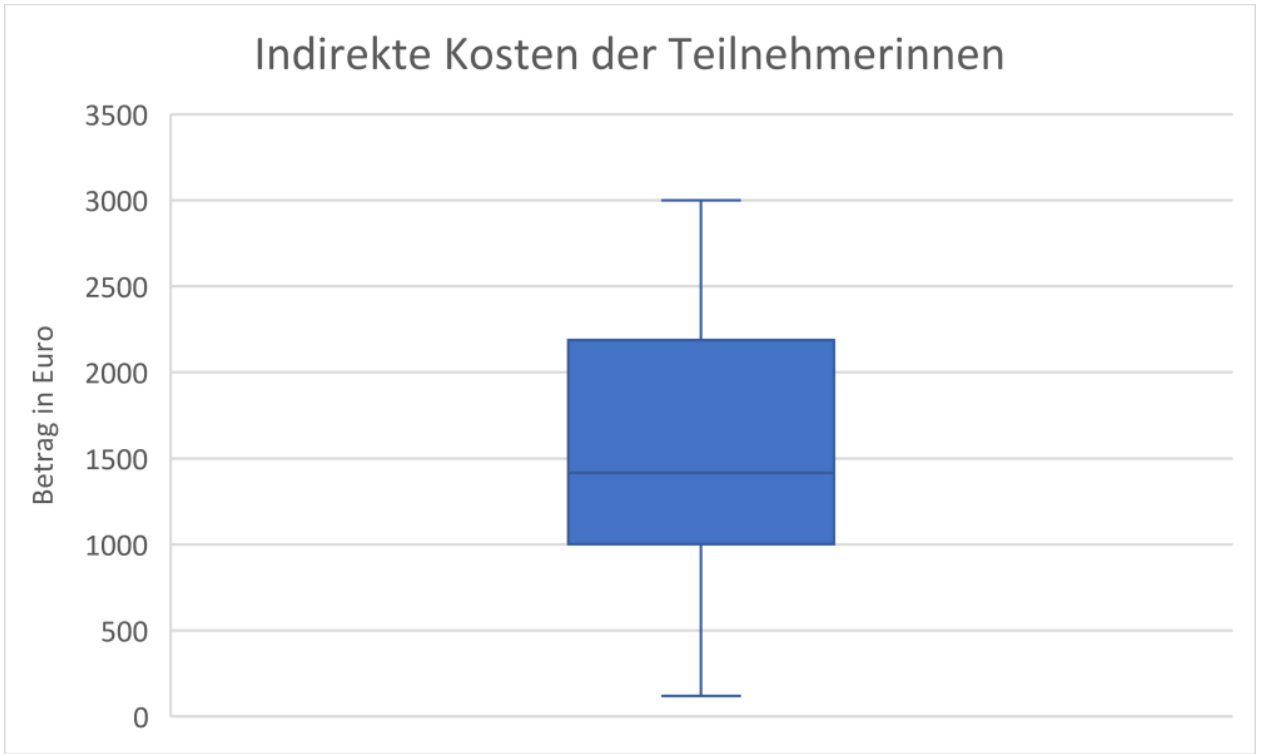




\subsection{Aufstellung der Gesamtkosten einer Geburt vor und nach Schließung der}

\section{stationären Geburtshilfe auf Föhr}

Tabelle 24: Aufstellung der Gesamtkosten einer Geburt vor und nach Schließung der stationären Geburtshilfe auf Föhr

\section{Entbindung in Föhr Entbindung auf dem Festland}

(Referenzjahr 2017)

\begin{tabular}{lrr}
\hline $\begin{array}{l}\text { Direkte nicht medizinische } \\
\text { Kosten der Teilnehmerinnen }\end{array}$ & $* 632 € /$ Familie \\
\hline $\begin{array}{l}\text { Direkte nicht medizinische } \\
\text { Kosten der Leistungsträger }\end{array}$ & $76.335 € / \mathrm{Jahr}$ & $116.230,86 € / \mathrm{Jahr}$ \\
\hline Direkte medizinische Kosten & $101.710,23 € / \mathrm{Jahr}$ & $107.403,17 € / \mathrm{Jahr}$ \\
\hline $\begin{array}{l}\text { Indirekte Kosten der } \\
\text { Teilnehmerinnen }\end{array}$ & $*$ & $1.416,25 € /$ Familie \\
\hline
\end{tabular}

* Daten nicht erhoben

Die Daten zu den direkten nicht medizinischen Kosten und den indirekten Kosten der Teilnehmerinnen vor der Schließung der Geburtshilfe auf Föhr wurden nicht erhoben, da es sich im Rahmen der Zielsetzung dieser Arbeit um vernachlässigbare Kosten handelt. Es kann davon ausgegangen werden, dass kaum Kosten dieser Art entstanden sind, weil die Teilnehmerinnen und deren Familien zeitlich und örtlich sehr flexibel waren und die Geburtsplanung und die Geburt besser in den Alltag integrieren konnten. Es entstanden zum Beispiel keine Fahrt- und Fährkosten oder Kosten zu einer außerordentlichen Freizeitgestaltung. Ein nennenswerter Verdienstausfall ist auch eher unrealistisch, da der Partner die Teilnehmerin nicht auf das Festland begleiten musste und somit seiner Arbeit nachkommen konnte. 


\section{Diskussion}

Im Rahmen der vorliegenden Dissertation wurden die anfallenden direkten und indirekten Kosten der Insulanerinnen auf Föhr, wenn vor Ort keine Geburtshilfe stattfindet, quantifiziert. Zudem wurden die direkten medizinischen und direkten nicht-medizinischen Kosten der Kostenträger erfasst, die im Zusammenhang mit der Schließung der Geburtshilfe auftreten. Die vorliegende Dissertation reiht sich nicht nur in die bestehende Literatur in diesem Kontext ein, sondern ermittelt erstmals auch die direkten nicht-medizinischen Kosten und die indirekten Kosten der betroffenen Familien, wenn keine wohnortnahe Geburtshilfe verfügbar ist.

Dies ist bisher die einzige Studie, die eine Kostensteigerung der direkten medizinischen und nichtmedizinischen Kosten nach Schließung einer geburtshilflichen Abteilung nachweisen kann. Unsere Studie zeigt eine Steigerung der direkten nicht-medizinischen Kosten um 34,3 Prozent. Weiterhin zeigt sie, dass die betroffenen Frauen einer erheblichen finanzieller Belastung ausgesetzt sind, wenn keine wohnortnahe Geburtshilfe möglich ist.

Die Ergebnisse der vorliegenden Dissertation werden zunächst im Hinblick auf die in der Einleitung vorgestellten Studienlage diskutiert. Darauffolgend werden die Ergebnisse in die aktuelle gesundheitspolitische Lage eingeordnet und die Stärken und Schwächen dieser Arbeit werden benannt. Abschließend erfolgt eine Zusammenfassung mit Schlussfolgerungen.

\subsection{Einordnung der Ergebnisse in die aktuelle Literatur}

Im Rahmen der Literaturrecherche fanden sich drei zentrale Forschungsgebiete, die für diese Dissertation von Relevanz sind: der medizinische, der psychosoziale und der gesundheitsökomische Aspekt der Zentralisierung der geburtshilflichen Versorgung.

Die vorliegende Arbeit kann dabei in das zuletzt genannte Forschungsgebiet eingeordnet werden und dort einen Beitrag leisten. Der medizinische Aspekt der Zentralisierung der geburtshilflichen Versorgung wird in dieser Dissertation nicht erforscht, muss aber trotzdem im Rahmen der gesundheitspolitischen Debatte beachtet werden.

Der psychosoziale Aspekt der Zentralisierung der geburtshilflichen Versorgung wurde in der aktuellen Dissertation nur angerissen, da dieses Gebiet im Rahmen einer zweiten Dissertation von einer weiteren Doktorandin intensiv erforscht wird.

Im Rahmen der Literaturrecherche wurde ein Fokus auf die gesundheitsökonomischen Faktoren gelegt. Augurzky et al. konstruierten eine gynäkologische und geburtshilfliche Basisklinik, die gerade noch kostendeckend arbeiten kann [9]. Diese Basisklinik weist eine Patientenstruktur von 485 
gynäkologischen und 1.115 geburtshilflichen Fällen, davon 432 Geburten auf. Gestützt auf solche Arbeiten lässt sich die Forderung der AOK nach einer Mindestfallzahl von 500 Geburten pro Jahr nachvollziehen [64]. Die Geburtshilfe auf Föhr kann und konnte offensichtlich nie diese Mindestfallzahl erreichen. Augurzky et al. betonen weiterhin, dass die soeben beschriebenen Berechnungen nicht für belegärztliche Häuser herangezogen werden können und dass „weiterer Forschungsbedarf in der Regionalisierung der Ergebnisse unter Berücksichtigung der tatsächlich existierenden Klinikstandorte und der regionalen Bevölkerungsdichte (besteht).“ [1]

Aus wirtschaftlicher Sicht ist die Geburtshilfe auf Föhr nicht oder nur durch erhebliche finanzielle Zuschüsse finanzierbar, allerdings ist der Standort aufgrund der regionalen Besonderheiten, speziell der Insellage, als Sonderfall zu betrachten. Durch die Schließung der Geburtshilfe fallen nämlich Kosten an, die vorher nicht von Relevanz waren. Hierbei sind die direkten medizinischen Kosten für die Entbindung vernachlässigbar. Diese unterscheiden sich nach den Berechnungen im Rahmen des DRG Systems nur um 5,6 Prozent pro Jahr und werden daher im Rahmen dieser Arbeit nicht weiter beleuchtet.

Zu beachten sind vorwiegend die direkten nicht-medizinischen Kosten der Leistungsträger, sowie der betroffenen Familien. Unsere Studie zeigt, dass nach der Schließung der geburtshilflichen Abteilung auf Föhr 116.230,86 Euro pro Jahr an direkten nicht-medizinischen Kosten der Leistungsträger entstehen. Diese Kosten werden getragen von den gesetzlichen und privaten Krankenversicherungen und dem Kreis Nordfriesland.

Die Krankenversicherungen übernehmen daher nun Kosten während der geburtshilflichen Versorgung, die vorher nicht bestanden. Hierbei handelt es sich um die Übernahme der Kosten für die Unterkunft in einer Boarding-Einrichtung, einem Pauschalbeitrag zum Hebammennotruf und den Kosten für die verschiedenen Rettungswege im Falle eines notfallmäßigen Transports auf das Festland. So entstehen den Krankenversicherungen insgesamt direkte nicht-medizinische Kosten in Höhe von 31.806,63 Euro jährlich für 56 Geburten.

Unsere Studie zeigt, dass neben den Kosten der Krankenkassen Kosten für die Landkreise, das Land Schleswig-Holstein und die Patientinnen/Familien entstehen:

Der Kreis Nordfriesland beteiligt sich unter anderem an den Kosten der geburtshilflichen Versorgung nach Schließung der Geburtshilfe. Er leistet einen Beitrag zum Hebammennotruf und übernimmt die Kosten der Haftpflichtversicherungen beider Hebammen, die in der Geburtshilfe tätig sind. Ebenso übernimmt er die Pauschale für die Rufbereitschaft der vor Ort tätigen Gynäkologin sowie einen Anteil an der Haftpflichtversicherung der Gynäkologin. Weitere Zuschüsse kommen seit dem 01.01.2018 auch vom Land Schleswig-Holstein. Diese waren zwar für die Berechnungen dieser Dissertation nicht 
relevant, da exemplarisch nur das Jahr 2017 betrachtet wurde. Der Kreis Nordfriesland hat demnach jährliche direkte nicht-medizinische Kosten von 72.634,23 Euro.

Auffällig ist in diesem Kontext, dass es eine Neu- und Umverteilung der Kosten gibt: Der Kreis Nordfriesland, der vorher nur indirekt über das Klinikum Nordfriesland in die Finanzierung der Geburtshilfe involviert war, tritt nun als neuer Akteur auf und übernimmt einen erheblichen Anteil der direkten nicht-medizinischen Kosten, vor allem die Finanzierung und damit Sicherstellung der geburtshilflichen Rufbereitschaften der Hebammen und der Gynäkologin auf Föhr. Das Klinikum Nordfriesland übernahm vor der Schließung die Kosten für die Sicherstellung des Hebammennotrufs, der Rufbereitschaft der Gynäkologin und gewährte einen maximalen Zuschuss von 20.000 Euro pro Jahr für die Haftpflichtversicherung der Belegärztin, die die Geburtshilfe betreute. Diese Kosten werden nach der Schließung umverteilt: Die Kosten für den Hebammennotruf werden nun auf die Krankenversicherungen sowie den Kreis Nordfriesland umverteilt, die Kosten für die Rufbereitschaft und einen Anteil an der Haftpflichtversicherung der Gynäkologin trägt der Kreis Nordfriesland allein. Hier werden nicht nur Kosten um- und neuverteilt, sondern auch Verantwortungsbereiche.

In mehreren Studien zu den wirtschaftlichen und sozialen Folgen der Zentralisierung $[33,34]$ wird angesprochen, dass den betroffenen Frauen und Familien zusätzliche finanzielle Belastungen entstehen. In der vorliegenden Dissertation werden nun anknüpfend an die bestehende Forschung die Kosten, die den Frauen entstehen, wenn es keine wohnortnahe Geburtshilfe mehr gibt, identifiziert und erstmals auch quantifiziert.

In der durchgeführten Umfrage wurden die Frauen um Angaben zu ihren angefallenen Verpflegungskosten, Fahrtkosten, Fähr- und Parkkosten, Unterkunftskosten und sonstigen Kosten gebeten. Die Familien bezifferten ihre Gesamtkosten, die im Rahmen der Geburt und des Aufenthalts auf dem Festland anfielen, im Durchschnitt auf 631,79 Euro. Hierbei nehmen die Transportkosten, also die Fahrtkosten inklusive der Fähr- und Parkkosten mit durchschnittlich 41,3 Prozent der Gesamtkosten den höchsten Anteil ein. Die Verpflegungskosten und die Unterkunftskosten werden etwa gleich mit durchschnittlich 25,3 Prozent der Gesamtkosten angegeben. Hierbei ist allerdings zu berücksichtigen, dass für Unterkunftskosten bei der Familie eine Übernachtungspauschale von nur 24 Euro gemäß §9 des Einkommenssteuergesetzes angenommen wurde.

Grzybowski und Kornelsen [65] machen in ihrer Forschung deutlich, dass vor allem sozial benachteiligte Familien mit limitierten finanziellen Ressourcen unter einer deutlichen finanziellen Belastung stehen. Das durchschnittliche Einkommen im Kreis Nordfriesland in Schleswig-Holstein, in dem auch die Insel Föhr liegt, beträgt laut der Einkommenssteuerstatistik 2013 der Lohn- und Einkommenssteuerpflichtigen des Statistischen Amtes für Hamburg und Schleswig-Holstein 34.459 
Euro, dies entspricht einem monatlichen durchschnittlichen Einkommen von 2.871,58 Euro (Ermittlung nach der Summe der sieben Einkunftsarten der Steuerpflichtigen unter Verminderung des Altersentlastungsbetrages ( $\S 24 a \mathrm{EStG}$ ) und den Entlastungsbetrag für Alleinerziehende (§ 24b EStG)) [66].

Wenn man davon ausgeht, dass nur ein Familienmitglied arbeitet, beträgt der Anteil, den die Familien laut dieser Dissertation dann für eine Geburt auf das Festland einmalig ausgeben rund 22 Prozent des monatlichen Einkommens. Für Familien, die wie auf Föhr häufig in der saisonabhängigen Tourismusbranche arbeiten, kann das eine hohe finanzielle Belastung bedeuten.

Roach und Downes [34] erörterten, dass die finanziellen Probleme der Familien hauptsächlich aus einem Einkommensverlust resultierten. Dieser Einkommensverlust entstünde durch die vorzeitige Reise zur Geburt auf dem Festland. In der vorliegenden Dissertation sollten die Frauen auch Angaben zu ihrem Einkommensverlust beziehungsweise dem Einkommensverlust des Partners machen. 27,6 Prozent der Teilnehmerinnen gaben dabei indirekte Kosten an, die aus Arbeits- und Produktionsausfällen resultierten Dabei war es in der Gesamtheit der Fälle der Partner, der den Einkommensverlust hatte. Nur zwei Teilnehmerinnen gaben auch für sich selbst indirekte Kosten an. Die Partner waren dabei allesamt selbständig, zum Beispiel als Landwirt mit einem Landwirtschaftsbetrieb, Gärtner mit einem Gärtnereibetrieb, Gastronom oder Metallbauer in einem selbstständig geführten Metallbaubetrieb. Die Interviews ergeben, dass die angesprochenen Einkommensverluste hauptsächlich daraus resultierten, dass der Betrieb für mehrere Tage schließen musste, da der werdende Vater seine Frau auf das Festland begleitet hat oder sie zumindest nach der Geburt besucht oder abgeholt hat. Der Mittelwert der indirekten Kosten beträgt 1.416,25 Euro. Dies ist nahezu die Hälfte des durchschnittlichen monatlichen Einkommens in Nordfriesland laut Einkommenssteuerstatistik 2013.

Die Ergebnisse von Roach können daher nicht nur qualitativ, sondern auch quantitativ belegt werden. Allerdings muss auch beachtet werden, dass nur 27,6 Prozent der Stichprobe überhaupt indirekte Kosten angaben. Für die restlichen Teilnehmerinnen der Dissertation war der Einkommensverlust kein relevanter Kostenparameter.

Ebenfalls scheint ein Kostenfaktor der Geburt auf dem Festland die Kategorie „sonstige Kosten“ zu sein. Hierunter fallen Kosten, die zur Freizeitgestaltung anfielen, wie zum Beispiel Besuche im Freizeitpark, im Schwimmbad, in Museen oder im Kino. Die Durchschnittskosten in dieser Kategorie betrugen insgesamt 75,18 Euro. Das Ergebnis ist unter dem Gesichtspunkt zu betrachten, dass die Teilnehmerinnen im Durchschnitt 9,55 Tage prästationär auf dem Festland verweilten und dort auch ihren neuen „Alltag“ gestalten mussten. Die Freizeitgestaltung musste häufig auch für den Partner und 
in manchen Fällen für weitere Kindern finanziert werden. Das sind Kosten, die zuhause nicht oder nicht in diesem Ausmaß entstanden wären. Dieser Kostenfaktor wurde in der bisherigen Literatur bislang kaum berücksichtigt.

Beachtlich sind auch die Ergebnisse zu den Verpflegungskosten von im Durchschnitt 155,89 Euro. Diese sind sowohl bei den Teilnehmerinnen, die das Boarding Konzept nutzten (durchschnittlich 147,50 Euro), als auch bei den Teilnehmerinnen, die bei der Familie wohnten (durchschnittlich 135 Euro) existent. Aus den Interviews geht hervor, dass in manchen Boarding-Häusern ein Frühstück und Mittagessen inkludiert ist, in anderen Einrichtungen jedoch nicht. Zudem war es in manchen Einrichtungen zu dem Zeitpunkt nicht möglich, selbst zu kochen, sodass die Familien gezwungen waren, essen zu gehen.

\subsection{Einordung der Ergebnisse in die aktuelle gesundheitspolitische Debatte}

Spätestens seit Schließung der Geburtshilfe auf Föhr ist eine regionale, aber auch landesweite gesundheitspolitische Debatte um die wohnortnahe Versorgung von Schwangeren entstanden. Die Ergebnisse sollen im Folgenden in diesem Zusammenhang eingeordnet werden.

Bereits in der Einleitung wurde das Gutachten „Maßnahmenkatalog zur Verbesserung der Situation der Geburtshilfe in Schleswig-Holstein" der OptiMedis AG, das vom Ministerium für Soziales, Gesundheit, Wissenschaft und Gleichstellung in Schleswig-Holstein in Auftrag gegeben wurde, erwähnt. In diesem Gutachten werden dem Land Schleswig-Holstein sechs Maßnahmen empfohlen, die die geburtshilfliche Situation verbessern könnten. Diese Maßnahmen umfassen unter anderem ein Partnerschaftsmodell Mutter-Hebamme-Arzt, eine intensivere und individuelle vorgeburtliche Betreuung, die Sicherstellung einer kontinuierlichen Hebammenversorgung, eine Optimierung der geburtshilflichen Notfallversorgung, eine Evaluation und Qualitätssicherung, sowie eine „Vernetzungsagentur Gesundheit“.

Das Ministerium für Soziales, Gesundheit, Wissenschaft und Gleichstellung des Landes SchleswigHolstein nahm Anfang 2017 Stellung zu dem Gutachten und nahm eine Priorisierung der Maßnahmen vor [67]. Hierbei fokussierte sich das Ministerium vor allem auf die Sicherstellung einer kontinuierlichen Hebammenversorgung, die Optimierung der geburtshilflichen Notfallversorgung und die Implementierung des Partnerschaftsmodells. Die Umsetzung der Maßnahmen kann man vor allem im Bereich der Hebammenversorgung erkennen. Rückwirkend zum 01.01.2018 wurde die 24 Stunden Rufdienstpauschale für die Hebammen auf 100 Euro erhöht, außerdem unterstützt das Land Schleswig-Holstein den Kreis Nordfriesland und das Amt Föhr/Amrum in der Deckung des Finanzierungsbedarfs der Hebammenversorgung mit jährlich 10.000 Euro. Ebenso wurde eine 
langfristige Finanzierungslösung für die gynäkologische-geburtshilfliche Rufbereitschaft gefunden, die ebenfalls der Kreis trägt. [53]

Aus diesem konkreten Beispiel wird ersichtlich, dass die verantwortlichen Akteure bemüht sind, die geburtshilfliche Situation finanziell in Schleswig-Holstein zu verbessern.

Allerdings fehlt in all den Bemühungen und Maßnahmenvorschläge die Beachtung der individuellen Situation der betroffenen Mütter und Familien. In den Ergebnissen dieser Dissertation wird deutlich, dass die Familien, abgesehen von psychosozialen Faktoren, durchschnittlich eine nicht zu unterschätzende finanzielle Last tragen müssen. Zu diesen finanziellen Problemen reihen sich außerdem organisatorische Herausforderungen. Das OptiMedis-Gutachten fordert unter anderem eine Evaluation und Qualitätssicherung der aktuellen Datenlage, um etwaige Qualitäts- und Kostenprobleme zu analysieren. Die Ergebnisse der vorliegenden Dissertation können zu dieser Analyse, zumindest auf der Basis der direkten nicht medizinischen Kosten der beteiligten Frauen, beitragen. Eine finanzielle Unterstützung der Familien sollte aufgrund der Ergebnisse auch in einem größeren Rahmen diskutiert werden.

Weiterhin kommt diese Dissertation zu dem Ergebnis, dass die direkten nicht-medizinischen Kosten der Leistungsträger nach der Schließung von 76.335 Euro auf 116.230,86 Euro steigen. Dies entspricht einer Steigerung von 34,3 Prozent und sollte in der aktuellen Debatte berücksichtigt werden. Die Kostensteigerung resultiert vor allem aus der Übernahme der Unterkunftskosten des BoardingAngebotes und der notfallmäßigen Rettungstransporte auf das Festland.

Es sind aber nicht nur die Politik und die Kostenträger, die versuchen, die geburtshilfliche Situation in Schleswig-Holstein zu verbessern; im Zuge der Schließung der Geburtshilfe auf Föhr bildete sich auch eine Initiative von Bürgerinnen und Bürgern, die sich zunächst für die Wiedereröffnung des Kreißsaales durch Öffentlichkeitsarbeit und Proteste einsetzte. 2017 wurde aus der Initiative "Inselgeburt" der eingetragene Verein Inselgeburt. Mit den dann weiter gefassten Zielen, unter anderem der Förderung der Autonomie von Schwangeren auf Föhr und Amrum sowie einer beratenden Funktion für werdende Mütter über Möglichkeiten und Grenzen der außerklinischen Geburt, nimmt der Verein eine nicht unerhebliche Stellung in der Betreuung der Schwangeren auf Föhr und Amrum ein.

Eine Bewertung dieses Konzeptes ist mit der vorliegenden Dissertation nicht möglich, es zeigt aber, dass die Debatte nicht nur die gesundheitspolitische Ebene bewegt, sondern auch die davon betroffene Bevölkerung.

Eine Übertragung der Ergebnisse auf ähnliche Fälle muss jedoch aufgrund der einzigartigen geographischen Lage der Insel Föhr genau geprüft werden. Ansätze dieser Dissertation lassen sich aber 
auch auf ähnliche Fälle auf dem Festland übertragen, da auch auf dem Festland teilweise weite Anfahrtswege zu Entbindungskliniken bestehen [68]. Ein weiterer wichtiger Forschungsansatz auf Basis dieser Dissertation könnte sein, eine Verlaufsstudie nach einer gewissen Zeit zu verfassen. Hier könnten die neuen Konzepte zur geburtshilflichen Versorgung auf ihre Auswirkungen geprüft werden.

\subsection{Stärken und Schwächen der Dissertation}

Der methodische Ansatz der Dissertation ist zweigeteilt in einen qualitativen und quantitativen Teil und bietet den Vorteil, dass Ergebnisse aus dem qualitativen Teil im quantitativen validiert oder verworfen werden konnten. Auch der stufenweise Prozess der Interview- sowie Umfragevorbereitung und -durchführung hat stets Raum für Anpassungen und Überprüfungen im Rahmen der methodischen Datenerfassung geboten.

Für die Kostenerfassung wurde mit einem Minimalkostenprinzip gearbeitet, sodass davon ausgegangen werden kann, dass die tatsächlichen Kosten eher unterschätzt und nicht überschätzt wurden. In dem Kapitel „Material und Methoden“ wurde dazu eine Beispielrechnung zu den minimalen Transportkosten vorgenommen, um das Minimalkostenprinzip zu verdeutlichen.

Eine Stärke dieser Arbeit ist, dass das subjektive und bisher weitgehend nur qualitativ bearbeitete Forschungsproblem wissenschaftlich und datenfundiert auf regionaler Ebene aufgearbeitet wurde. Die zeitliche und räumliche Nähe zum Forschungsumfeld haben dazu beigetragen, eine Dissertation zu erarbeiten, die in den aktuellen gesundheitspolitischen Diskurs eingebettet ist und somit einen wissenschaftlichen Beitrag zu der Debatte leisten kann.

Eine Schwäche dieser Arbeit ist die niedrige Rücklaufquote (26,13 Prozent). Allerdings ist zu berücksichtigen, dass die Grundgesamtheit, also die Anzahl aller auf Föhr lebenden und gemeldeten Frauen, die ein Kind zwischen dem 01. Oktober 2015 und dem 30. September 2017 auf dem Festland geboren haben, ohnehin schon klein ist. Hier kann, wie im Ergebnisteil erörtert, von einer Grundgesamtheit von circa 111 Frauen ausgegangen werden. Somit handelt es sich um eine regionale Analyse, deren Übertragung auf einen größeren Kontext genau geprüft werden sollte.

Eine weitere Schwäche der Arbeit ist, dass nur Durchschnittskosten berücksichtigt und Annahmen zum Ressourcenverbrauch und der monetären Bewertung der Ressourcen getroffen werden mussten, da von den beteiligten Akteuren oft keine Angaben zur monetären Bewertung der Ressourcen gemacht wurden - trotz intensiver Nachfrage. Im Laufe der Recherche zeigte sich in Bezug auf die objektive Datenerhebung oft eine große Intransparenz, die es schwer gemacht hat, an verwertbare und repräsentative Daten zu gelangen. Dies allein kann aber auch als Rechercheergebnis gewertet werden: 
Das Gesundheitswesen im untersuchten Fall ist von Intransparenz geprägt. Eine Offenlegung von Finanz- und Kostenstrukturen ist selbst für wissenschaftliche Zwecke offenbar nicht von allen erwünscht.

Die Kostenerfassung, insbesondere der beteiligten Familien, ist inkomplett, da viele Frauen ein Netzwerk von Familie und Freunden genutzt haben, um die Geburt auf dem Festland zu organisieren. Die Kosten des „Netzwerks“, wie zum Beispiel der Großeltern oder der Geschwister und Freunde, konnten in dieser Dissertation nicht quantifiziert werden. Während die fehlende Quantifizierung in dieser Dissertation als Limitation gewertet werden könnte, zeigt sich hier auch eine Anknüpfungsmöglichkeit für zukünftige Forschung: Die Auseinandersetzung mit dem informellen Netzwerk und dessen Kosten sollte in den Diskurs mit aufgenommen werden.

\section{Zusammenfassung mit Schlussfolgerungen und Empfehlungen}

Ausgangspunkt dieser Forschungsarbeit war die Schließung der geburtshilflichen Abteilung auf der nordfriesischen Insel Föhr zum 1. Oktober 2015. Die Schließung dieser Abteilung steht im Zusammenhang mit einem größeren Themenkomplex um die Zentralisierung und Spezialisierung im Gesundheitswesen in Deutschland. Das Ziel der Arbeit war, die Schließung der Geburtshilfestation auf Föhr in ihrem gesundheitsökonomischen Kontext zu betrachten. Dafür wurde eine Aufarbeitung der Schließung der Geburtshilfestation auf Föhr in mehreren Schritten unternommen.

Es wurden die anfallenden direkten und indirekten Kosten der Insulanerinnen quantifiziert und die direkten medizinischen und direkten nicht-medizinischen Kosten der Kostenträger erfasst, die im Zusammenhang mit der Schließung der Geburtshilfe auftraten.

Hier zeigte sich, dass die durchschnittlichen direkten nicht-medizinischen Kosten der Insulanerinnen 631,79 Euro insgesamt betrugen. Die Fahrtkosten inklusive Fähr- und Parkkosten hatten daran den größten Anteil. 27,6 Prozent der Teilnehmerinnen gaben auch indirekte Kosten an. Der Mittelwert der indirekten Kosten betrug insgesamt 1.416,25 Euro.

Die direkten nicht-medizinischen Kosten der Kostenträger betrugen nach Schließung der Geburtshilfe 116.230,86 Euro unter der Annahme von jährlich 56 Geburten.

Die direkten medizinischen Kosten nach Schließung der Geburtshilfe betrugen 107.403,17 Euro pro Jahr.

Im Vorher-Nachher-Vergleich konnte ein Anstieg der direkten nicht-medizinischen Kosten um 34,3 Prozent nachgewiesen werden. Außerdem konnte gezeigt werden, dass eine Umverteilung der Kosten 
zwischen den Kostenträgern stattgefunden hat und dementsprechend auch Verantwortungen neu verteilt wurden. Ein wichtiger Akteur in der geburtshilflichen Versorgung nach Schließung der geburtshilflichen Station auf Föhr ist der Kreis Nordfriesland mit dem Land Schleswig-Holstein im Hintergrund. Bei den direkten medizinischen Kosten konnte kein nennenswerter Unterschied zwischen Vorher und Nachher festgestellt werden.

Der größte Fokus zur Verbesserung der Versorgungssituation sollte den Familien zukommen, da sie laut den Ergebnissen dieser Dissertation einer nicht unerheblichen finanziellen Belastung ausgesetzt sind. Einige Krankenkassen gewähren bereits Fahrtkostenzuschüsse im Rahmen der Geburt. Außerdem können Schwangere gemäß § 24h SGB V eine Haushaltshilfe beantragen. Die bestehenden Angebote sollten transparenter kommuniziert und ausgebaut werden. Hier könnte man zum Beispiel einen rechtlichen Anspruch auf Fahrtkostenzuschüsse diskutieren.

In diesem Rahmen sollte auch das Boarding Konzept so optimiert werden, dass es für alle Standorte die gleichen Standards bietet, sodass für alle Familien gleiche (finanzielle) Voraussetzungen herrschen, ungeachtet dessen für welchen Standort sie sich entscheiden. Hierdurch könnte die finanzielle Belastung der Familien gesenkt werden.

Leider wurden wesentliche Informationen von den Kostenträgern vor allem zu der monetären Bewertung der Ressourcenverbräuche verweigert. Die Intransparenz in diesem Kontext stellt sich, nach intensiver Auseinandersetzung mit diesem Thema, als ein großes Hindernis in der Weiterentwicklung der Konzepte zur geburtshilflichen Versorgung in Schleswig-Holstein dar. Es wäre für die Sicherheit und Versorgung der Schwangeren und Neugeborenen wünschenswert, dass die beteiligten Akteure zusammenarbeiten und auch ein Datenaustausch stattfindet, um Lücken und Schwächen der Versorgung aufzudecken. Hierzu könnten prospektive Daten erhoben werden.

Aufgrund der Erläuterungen in Abschnitt 4.1.1 könnten aus finanzieller Sicht Maßnahmen unterstützt werden, die die Kosten für die geburtshilflichen Notfallrettungswege minimieren und somit einen großen Anteil der direkten nicht-medizinischen Kosten senken könnten. Das schließt erstens eine individuelle und risikoadaptierte geburtshilfliche Vorsorge der Frauen ein, um rechtzeitig Notfälle zu erkennen und unnötige Rettungsfahrten zu vermeiden. Und zweitens ist auch die Maßnahme zu befürworten, Rettungspersonal zu geburtshilflichen Notfällen zu schulen, um die Sicherheit von Kind und Mutter zu gewährleisten.

Die Kostenerfassung dieser regionalen Analyse zeigt gesundheitsökonomische Perspektiven auf, indem sie deutlich macht, dass einerseits die betroffenen Frauen mit dem umgebenen Netzwerk an 
Familie und Freunden einer erheblichen finanziellen Belastung ausgesetzt sind und andererseits neue und mehr direkte nicht-medizinische Kosten auf den Kostenträgern lasten. Diese beiden wichtigen Ergebnisse sollten in der gesundheitspolitischen Debatte Beachtung finden und als Motivation genommen werden, die angeschobenen Konzepte zu stärken und zu erweitern. 


\section{Literatur}

1 Statistisches Bundesamt. Grunddaten der Krankenhäuser. Fachserie 12; Reihe 6.1.1., 1991-2016

2 Gesundheitsberichterstattung des Bundes (GBE). Entbindungen in Krankenhäusern. Online: http://www.gbe-bund.de/oowa921-

install/servlet/oowa/aw92/dboowasys921.xwdevkit/xwd_init?gbe.isgbetol/xs_start_neu/\&p_aid $=i \& p \_a i d=1040083 \&$ nummer $=552 \& p \_s p r a c h e=D \& p \_i n d s p=-\& p \_a i d=10855736$ letzter Zugriff: 21.03.2018

3 Pötzsch O, Rößger F. Bevölkerung Deutschlands bis 2060. 13. koordinierte Bevölkerungsvorausberechnung; 2015

4 Bundesinstitut für Bevölkerungsforschung. Geburtenentwicklung nach Kreisen, 1990-2015

5 Richtlinie über Maßnahmen zur Qualitätssicherung der Versorgung von Früh- und Reifgeborenen gemäß §136 Absatz 1 Nummer 2 SGB V in Verbindung mit §92 Abs. 1 Satz 2 Nr. 13 SGB V. Bundesanzeiger; 2005

6 S1 Leitlinie 015/078: Mindestanforderungen an prozessuale, strukturelle und organisatorische Voraussetzungen für geburtshilfliche Abteilungen der Grund- und Regelversorgung; 2013

7 Schleußner E. 204. Stellungnahme der DGGG: Zur Anfrage "Expertenanhörung zum Thema Geburtshilfe" des Ministeriums für Soziales, Gesundheit, Wissenschaft und Gleichstellung Schleswig-Holstein; 2014

8 Litsch M. Mehr Mut zur Umsetzung von Mindestmengenregelungen nötig; 28.02.2017

9 Augurzky B, Krolop S, Römer K et al. Allgemeine Gynäkologie. Frauenheilkunde und Geburtshilfe in Deutschland - Kosten und Erlöse. Geburtshilfe und Frauenheilkunde 2009; 69: 560-563

10 Augurzky B, Krolop S, Römer K et al. Frauenheilkunde und Geburtshilfe. Frauenheilkunde und Geburtshilfe in Deutschland - Mindestgröße und Bedarf an Kliniken. Geburtshilfe und Frauenheilkunde 2010; 70: 402-405

11 Gesetzliche Krankenversicherung Spitzenverband. Hebammen (07.09.2017). Online: https://www.gkvspitzenverband.de/krankenversicherung/ambulante_leistungen/hebammen/hebammen.jsp letzter Zugriff: 20.10.2018

12 Bundesministerium für Gesundheit. Antwort der Bundesregierung auf die Kleine Anfrage der Abgeordneten Birgit Wöllert, Cornelia Möhring, Sabine Zimmermann (Zwickau), weiterer Abgeordneter und der Fraktion DIE LINKE.; 02.05.2017

13 Deutsches Ärzteblatt. Haftpflichtprämien von Belegärzten in der Geburtshilfe überschreiten 60.000-Euro-Grenze. Online:

https://www.aerzteblatt.de/nachrichten/83114/Haftpflichtpraemien-von-Belegaerzten-in-derGeburtshilfe-ueberschreiten-60-000-Euro-Grenze letzter Zugriff: 01.11.2018

14 Soergel $P$, Hillemanns $P$, Kaisenberg $C$ von. Haftpflicht: Zwei-Klassen-Haftung in der Geburtshilfe. Deutsches Ärzteblatt 27.03.2015; 112

15 Hibbeler D. Haftpflichtprämie. Geburtshilfe in Gefahr. Deutsches Ärzteblatt 21.03.2014; 111: 490

16 Ministerium für Soziales, Gesundheit, Familie und Gleichstellung. Situation und Perspektiven der Geburtshilfe in Schleswig-Holstein; 16.06.2014

17 Teilabschrift Medizinischer Teil. Gutachten Geburtshilfe Föhr; 2016

18 Ministerium für Soziales, Gesundheit, Wissenschaft und. Versorgung von Schwangeren und Neugeborenen in Schleswig-Holstein. Derzeitige Situation und Zukunftsperspektiven; 08.08.2015

19 Klinikum Nordfriesland. Geburtshilfe auf der Insel Föhr schließt Ende November 2015; 15.09.2015

20 Hansen $K-H$. Anfrage zur beabsichtigten Schließung der Geburtshilfe in Wyk auf Föhr; 01.10.2015

21 Harrsen D. Schließung der Geburtshilfe im Klinikum Wyk auf Föhr; 20.10.2015 
22 Klinikum Nordfriesland. Nach Schließung der Geburtshilfe: Abstimmung eines Versorgungskonzeptes für die Schwangeren der Insel Föhr auf einem guten Weg; 02.10.2015

23 Engel J., Holter T. Ihre Bitte um Stellungnahme zur Drucksache 18/3338, Anhörung vor dem Sozialausschuss des Landtages SH. 18. Aufl.; 17.01.2016

24 Elger K. Wehe! Der Spiegel 03/2017: 108-109

25 Hahn T. Kostendruck, fehlende Hebammen, steigendes Haftungsrisiko: Immer mehr Geburtsstationen auf dem Land müssen schließen. Auf Föhr rebellieren nun die Einwohner. Süddeutsche Zeitung 05.10.2016

26 Hochreither I. Der letzte Insulaner. Der Stern 19.05.2016: 119-121

27 Lasswell SM, Barfield WD, Rochat RW et al. Perinatal regionalization for very low-birth-weight and very preterm infants. A meta-analysis. JAMA 2010; 304: 992-1000

28 Merlo J, Gerdtham U-G, Eckerlund I et al. Hospital level of care and neonatal mortality in lowand high-risk deliveries. Reassessing the question in Sweden by multilevel analysis. Medical care 2005; 43: 1092-1100

29 Tracy SK, Sullivan E, Dahlen H et al. Does size matter? A population-based study of birth in lower volume maternity hospitals for low risk women. BJOG : an international journal of obstetrics and gynaecology 2006; 113: 86-96

30 Heller G, Richardson DK, Schnell $R$ et al. Are we regionalized enough? Early-neonatal deaths in low-risk births by the size of delivery units in Hesse, Germany 1990-1999. International Journal of Epidemiology 2002: 1061-1068

31 Grytten J, Monkerud L, Skau l et al. Regionalization and local hospital closure in Norwegian maternity care--the effect on neonatal and infant mortality. Health services research 2014; 49: 1184-1204

32 Kornelsen J, Grzybowski S. Safety and community. The maternity care needs of rural parturient women. Journal of obstetrics and gynaecology Canada: JOGC 2005; 27: 554-561

33 Kornelsen J, Stoll K, Grzybowski S. Stress and anxiety associated with lack of access to maternity services for rural parturient women. The Australian journal of rural health 2011; 19: 9-14

34 Roach SM, Downes S. Caring for Australia's most remote communities. Obstetric services in the Indian Ocean Territories. Rural and remote health 2007; 7: 699

35 Augurzky B, Kreienberg R, Mennicken R. Zukunft der stationären Versorgung in Gynäkologie und Geburtshilfe. Der Gynäkologe 2015; 48: 495-500

36 Geraedts M, Siegrist J. Gesundheitsökonomie in der Gynäkologie. Gynäkologe 2005; 38: 385-386

37 Kelle $U$. Die Integration qualitativer und quantitativer Methoden in der empirischen Sozialforschung. Theoretische Grundlagen und methodologische Konzepte. 2. Aufl. Wiesbaden: VS Verlag für Sozialwissenschaften / GWV Fachverlage GmbH Wiesbaden; 2008

38 Mayring P. Qualitative Inhaltsanalyse. Grundlagen und Techniken. 12. Aufl. Weinheim: Beltz; 2015

39 Fleßa S, Greiner W. Grundlagen der Gesundheitsökonomie. Eine Einführung in das wirtschaftliche Denken im Gesundheitswesen. 3. Aufl. Berlin, Heidelberg, s.l.: Springer Berlin Heidelberg; 2013

40 Schoffski O. Gesundheitsökonomische Evaluationen. Dordrecht: Springer; 2012

41 Schulenburg JM, Greiner W. Gesundheitsökonomik. 2. Aufl. Tübingen: Mohr Siebeck; 2012

42 Oberender P, Ecker T. Grundelemente der Gesundheitsökonomie. Bayreuth: P.C.O.-Verl.; 2001

43 Zapp W, Terbeck J. Kosten- versus Erlösverteilung im DRG-System. Wiesbaden: Springer Fachmedien Wiesbaden; 2014

44 Graf von der Schulenburg J-M, Greiner W, Jost F et al. Deutsche Empfehlungen zur gesundheitsökonomischen Evaluation - dritte und aktualisierte Fassung des Hannoveraner Konsens. Gesundheitsökonomie \& Qualitätsmanagement 2007; 12: 285-290 
45 Amtsverwaltung Föhr-Amrum. Neufassung der Entgeltordnung für den Städtischen Hafenbetrieb. 001537. Aufl.; 22.09.2009

46 Bundesrepublik Deutschland. Einkommenssteuergesetz $\S 9$ Werbungskosten. EStG

47 Schleswig-Holstein. Rettungsdienstgesetz §8; 2012

48 Kühn, M., Assistent der DGzRS, Bremen. Rettungskosten mit dem Seenotrettungsschiff (06.04.2018); 06.04.2018

49 Jötten F. Hubschrauber ans Bett. Weil es auf dem Land an Ärzten fehlt, kommt die Hilfe mehr und mehr aus der Luft. Ist das sinnvoll? Zeit Online 03.03.2011

50 Martens-Petersen H, Techniker Krankenkasse. Studie zu Geburtserfahrungen und -kosten von Insulanerinnen (02.05.2018); 02.05.2018

51 Kreis Nordfriesland, Fachbereich Jugend, Familie und Bildung. Kostenaufstellung Hebammenversorgung Sylt und Föhr. Husum; 21.03.2018

52 Kreis Nordfriesland. Beratung und Beschlussfassung über die Rufbereitschaftspauschale der Hebammen auf Föhr und Sylt; 23.03.2018

53 Kreis Nordfriesland. Beschluss aus der Niederschrift über die Sitzung des Kreistages des Kreises Nordfriesland. Beratung und Beschlussfassung über die Bezuschussung von gynäkologischen Bereitschaftsdiensten und Haftpflichtversicherungsprämien für die Insel Föhr; 17.11.2017

54 Nilvér H, Begley C, Berg M. Measuring women's childbirth experiences. A systematic review for identification and analysis of validated instruments. BMC pregnancy and childbirth 2017; 17: 203

55 Helfferich C. Die Qualität qualitativer Daten. Manual für die Durchführung qualitativer Interviews. 4. Aufl. Wiesbaden: VS Verlag für Sozialwissenschaften / Springer Fachmedien Wiesbaden GmbH Wiesbaden; 2011

56 Lamnek S. Qualitative Sozialforschung. Lehrbuch; mit Online-Materialien. 5. Aufl. Weinheim: Beltz; 2010

57 Flick U, Hrsg. Handbuch qualitative Sozialforschung. Grundlagen, Konzepte, Methoden und Anwendungen. München: Psychologie-Verl.-Union; 1991

58 Sjetne IS, Iversen $H$, Kjøllesdal JG. A questionnaire to measure women's experiences with pregnancy, birth and postnatal care. Instrument development and assessment following a national survey in Norway. BMC pregnancy and childbirth 2015; 15: 182

59 Baur N, Blasius J, Hrsg. Handbuch Methoden der empirischen Sozialforschung. Wiesbaden: Springer VS; 2014

60 Gusy B, Marcus K. Online Befragungen: Eine Alternative zu paper- pencil Befragungen in der Gesundheitsberichterstattung bei Studierenden [Schriftenreihe des AB Public Health: Prävention und psychosoziale Gesundheitsforschung Nr.01/P12]. Berlin: Freie Universität Berlin; 2012

61 Kölschbach P. Kreißsaal interessiert Forscher. Umfrage der Kieler Uni: Wie ergeht es Föhrerinnen, die auf dem Festland entbinden mussten? Der Insel-Bote 27.01.2018: 9

62 Kreis Nordfriesland. Studie der Universität Kiel zu "Geburtserfahrungen und -kosten von Insulanerinnen nach Schließung der stationären Geburtshilfe auf Föhr"; 07.08.2018

63 Von der Becke, C., Geschäftsführer Klinikum Nordfriesland. Studie der Universität Kiel zu "Geburtserfahrungen und -kosten von Insulanerinnen nach Schließung der stationären Geburtshilfe auf Föhr; 24.08.2018

64 Klauber J, Geraedts M, Friedrich J et al., Hrsg. Krankenhaus-Report 2017. Schwerpunkt: Zukunft gestalten. Stuttgart: Schattauer; 2016

65 Grzybowski S, Kornelsen J. The Costs of separation. The birth experiences of women in isolated and remote communities in British Columbia: The University of British Columbia; 2005

66 Statistisches Amt für Hamburg und Schleswig-Holstein. Lohn-und Einkommensteuerstatistik Schleswig-Holstein 2013. Große regionale Unterschiede; 11.10.2017 
67 Ministerium für Soziales, Gesundheit, Familie und Gleichstellung. Schleswig-Holsteinischer Landtag Umdruck 18/7539; 07.03.2017

68 Mennicken R, Kolodziej I, Augurzky B et al. Concentration of gynaecology and obstetrics in Germany. Is comprehensive access at stake? Health policy (Amsterdam, Netherlands) 2014; 118: 396-406 


\section{Anhang}

\section{Interviewleitfaden}

\begin{tabular}{|c|c|}
\hline & Begrüßung \\
\hline & 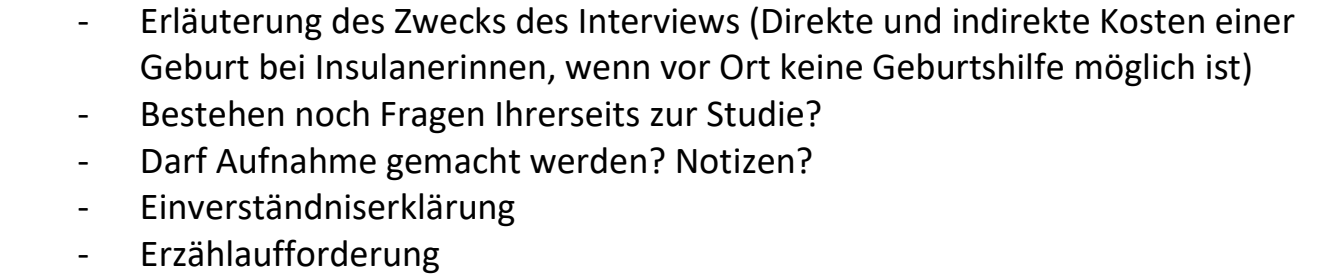 \\
\hline \multirow[t]{2}{*}{1.} & Soziodemographie \\
\hline & $\begin{array}{ll}\text { - } & \text { Alter } \\
\text { - } & \text { Familienstand } \\
\text { - } & \text { Das wievielte Kind wurde geboren? } \\
\text { - } & \text { Berufsausbildung und Berufstätigkeit vor Entbindung } \\
\text { - } & \text { Hilfen im Haushalt und bei der Kinderbetreuung? } \\
\text { - } & \text { Berufstätigkeit des Ehemannes/Partners }\end{array}$ \\
\hline \multirow[t]{2}{*}{2.} & Eckpunkte vor der Geburt \\
\hline & $\begin{array}{ll}\text { - } & \text { Probleme während der Schwangerschaft } \\
\text { - } & \text { Empfehlung der Gynäkologin/Hebamme bezüglich der Geburt } \\
\text { - } & \text { Vorstellungen und Wünsche bezüglich des Ablaufs der Geburt }\end{array}$ \\
\hline \multirow[t]{2}{*}{3.} & Wie es tatsächlich war \\
\hline & $\begin{array}{ll}\text { - } & \text { Zeitpunkt des Aufbruchs zum Festland } \\
\text { - } & \text { Wen war an der Entscheidung beteiligt? } \\
\text { Transport? } & \\
\text { - } & \text { Ggf. Übernahme der mütterlichen Aufgaben durch wen? Dauer, } \\
\text { übernommene Aufgaben? } \\
\text { - Inanspruchnahme des Boarding-Hauses: Wie war es da? Durfte jemand mit? } \\
\text { War jemand mit? Kosten? Organisation von welchem Leistungsträger (ggf. } \\
\text { Rolle der Versicherung)? Länge des Aufenthalts? }\end{array}$ \\
\hline \multirow[t]{2}{*}{4.} & Die größten Probleme rund um die Geburt \\
\hline & $\begin{array}{ll}- & \text { emotional } \\
\text { - } & \text { finanziell } \\
\text { - } & \text { organisatorisch }\end{array}$ \\
\hline \multirow[t]{2}{*}{5.} & Perspektive \\
\hline & $\begin{array}{ll}- & \text { Vorstellung von der nächsten Geburt? } \\
\text { - } & \text { Sicherheit oder/und Nähe zum Wohnort? }\end{array}$ \\
\hline
\end{tabular}




\section{Schließung der Geburtshilfestation auf Föhr}

Geburteritahrungen und togten von inaulanerinnen

nach achlielung der atatondren Geburtahlse auf Fohr

Uebe Fonrer Motter,

vielen Dank Aor Itre Beretachat, an dieger Umfrape telizunehmen.

Vorab noch ein pasr informatonen zum Fragebogen:

- Zum Auselilen des Onllnefrapebogens benotigen ale etwa 20 Mnuten

- Es geht stets um de Erfahrungen rund um dle Geburt Ihres ercton Kindes, weiches in dem Zetraum

nach der Schleßung der Geburtahifegtation aut Forr am 01 . Okbober 2015 bls zum 30 . September 2017 geboren wurde. We tere Kinder, de in desem Zeltraum geboren wurden, bielben von der studle

unberockalchtigt.

Die Kosten, nach denen ale in dem Fragebogen gefragt werden, betreffen nur die Koaten, de nicht von

der Krankenkasse abernommen wurden

Fre Antworten werden selbatveratandich anonymiglert

Diese Umfroos enthat 64 Fison.

\section{Allgemeines}

DIhr Alter (zum Zeitpunkt der Geburt Thres ersten Kindes nach Schliebung der Geburtshilfestation auf Fohr) *

In dieses Feld dirfen nur Zavien eingogeben wenden

Gne geben Bie thre Antwort hier ein.

[]Familienstand (zum Zeitpunkt der Geburt Ihres ersten Kindes nach SchlieBung der Geburtshilfestation auf Föhr) *

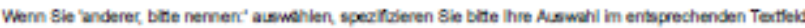

Bne wahien Bie nur eine der folgerden Avowoten aus

0 verheiratet

in einer festen Partnerschat

0 iedla

0 snderes, bitte nennen: 
[]Thr ausgeübter Beruf *

Bate geben 8 ie thre Avtront hier ein

[

Ausgeubter Beruf des Partners

Beantworten Sie diese Frage mut, worn folgende Bedigungen enfult sind: Fo

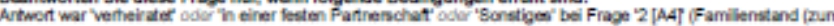

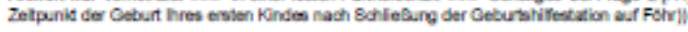

Bue geben 8 ie thre Actwont thier ein

-

[]Das wievielte Kind haben Sie bekommen? *

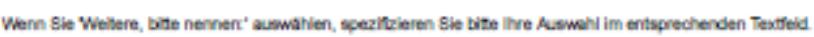

Bate whilien Bie nur eine der folgenden Avworten as

$O$ des Erste

dos Zweits

das Drtte

Weltere, btte nennen:

[]Geburtsmonat und -jahr des ersten Kindes nach Schliebung der Geburtshilfestation (z.B. Mai 2016)

ane geben 8 ie thre Antwont hier ein.

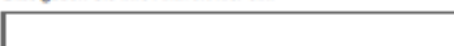

[II weicher Schwangerschaftswoche haben Sie Ihr Kind geboren? (z.B. $37+5=$ 38. SSW) *

Gne geten 8 ie thre Avtwort tier ent

[]Wie war Ihr Versichertenstatus zum Zeitpunkt der Geburt Ihres ersten Kindes nach Schließung der Geburtshilfestation? *

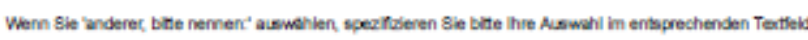

Bre wahien Bie nur eine der fogerden Avoworten aus

gesetzilch versicher

privat veralchert

0 snderes, bitte nennen: 


\section{Schwangerschaft}

[]Bitte geben Sie an, ob es in dieser Schwangerschaft Besonderheiten gegeben hat. *

Bane whien Ble die zatreffende Antwort tarjacien Punkt ass:

Zustand nach Kalserschnitt

Ja

Zustand nach Fruhgeburt ene:

vorthergen Kindes (Geburt vor

der 37 .

Schwangerschatewoche)

Obeket in der

Frohachwangerschat, die einen

krankenhausaufenths

notwendlg machte

Zwillings-bzN.

Mehringsachwangerschat

Blutungen nach dem 3.
Schwangerachatemona

Schwangerychahtad abete:

Schwangerrchatabluthochdruck

VorzeIge Wehentstigket

VorzeIIger Blszenaprun

- bevor eine regeimsige

Wehentatigkel auffrat)

Andere

Nein

Q

0

0

$Q$

Q

0

0

O

[]Bitte nennen Sie die "anderen" Besonderheiten Ihrer Schwangerschaft

Beantworten Sie diese Frage mut, wont folgende Bedingungen erfult sind:

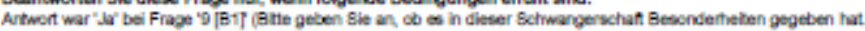
(Ansere),

Gate geben Sie thre Antwort hier ein

[ $\mathrm{Ab}$ wann in der Schwangerschaft haben Sie den Aufenthalt auf dem Festland zur Entbindung geplant? *

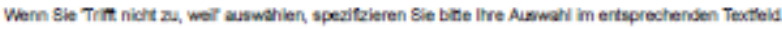

Bate whilen Ble nur eine der folgenden Avewoten aus:

0.-3. Monat

4.-5. Monst

7-9. Monat

Gar nicht

Q Trim nicht $z u$, weil 


\section{Unterkunft}

[]In welcher Stadt haben Sie entbunden? *

Bne geben 8ie thre Antwort tier ein

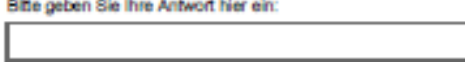

[]Wie einfach/schwierig war das Finden einer Unterkunft auf dem Festland für die Zeit vor der Entbindung? *

ghe wahien Sie die zatreffende Antwort tur josion Punkt as:

\begin{tabular}{|c|c|c|c|}
\hline echwierlo & $\begin{array}{c}\text { schwierlo } \\
Q\end{array}$ & $\begin{array}{c}\text { enfach } \\
Q\end{array}$ & senr einfach \\
\hline
\end{tabular}

[DDie vorherige Frage trifft nicht $\mathbf{z u}$, weil *

Beantwortion Sie diese Frage mu, wonn folgende Bedirgungen erfuit sind

Artwort war 'nicht satrefend' bei Frage '13 [C2] (We einfochsochwierig war das Finden einer Unterkurt auf dem Festland ar de zet vor der Entindung?

Bne geben 8 ie thre Avtwont hier ent.

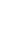

[]Wo waren Sie auf dem Festland untergebracht? *

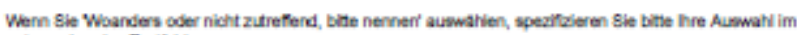
entaprechenden Tettiold

Bate wahien Sie nur dine der folgenden Avtwotan ass

Boarding Haus

Hote

Ferienwornung

Woanders oder nicht zutreffend, butte nennen 


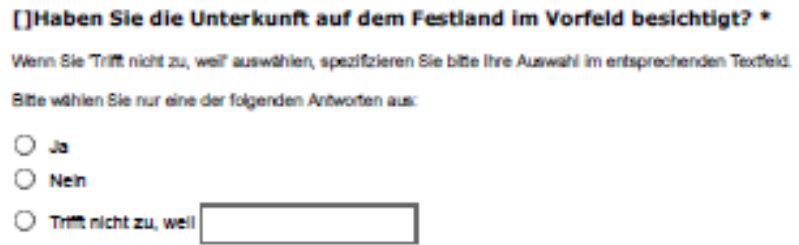

$\square$ Fashre

$\square$ Zug

$\square$ Bur

$\square$ sonatiges:

[]Haben Sie die Entbindungsklinik auf dem Festland im Vorfeld besichtigt? *

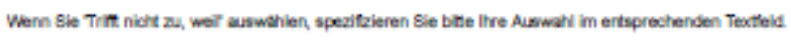
Bne whilen Sie nur dine der folgenden Avowoten as

0 da

Nen

Trim nicht $z u$, well

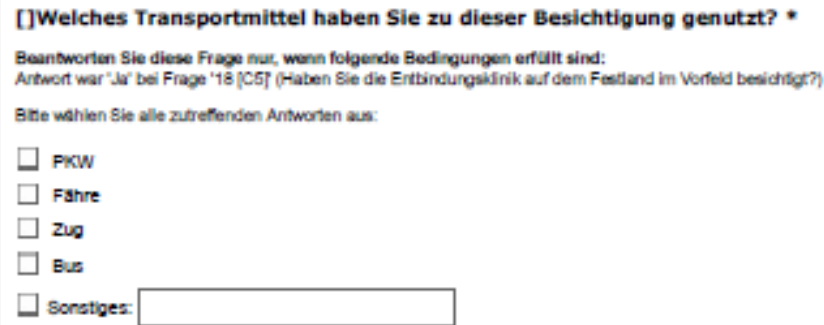


[]Wie viele Besichtigungsfahrten (1 Fahrt= Zuhause- Besichtigungsort- Zuhause) haben Sle vor der Geburt ingesamt unternommen? *

In dieses Feld dirfen nur Zanien eingegeben werden

Bue geben Bie lhe Antwort hier ein:

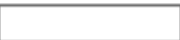

[]Wie beurteilen Sie die Sauberkeit Ihrer Unterkunft?

gne wahien Sie die zutreftende Antwont for josien Punkt as

$\begin{array}{ccccc}\begin{array}{c}\text { sehr } \\ \text { schmutzig }\end{array} & \text { schmutzig } & \text { sauber } & \text { sehr saber } & \text { mitreftend } \\ 0 & 0 & 0 & 0 & 0\end{array}$

[]Die vorherige Frage trifft nicht zu, weil *

Beantworten Sie diese Frage mus, wonn folgende Bedingungen erfult sind:

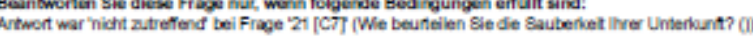

Gne geben Bie line Antwort hier ein.

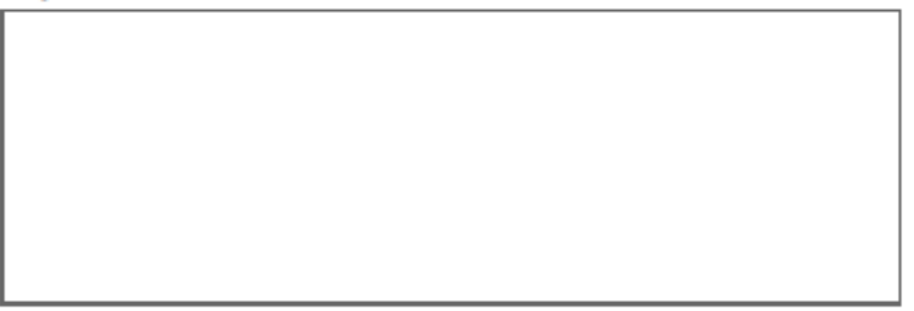

[]Wie beurteilen Sie die GrbBe Threr Unterkunft? *

Bane whilen Bie de zitreftende Antwort fur josten Punkt as:

$\begin{array}{ccccc}\text { zueng } & \text { eng } & \text { anpemessen } & \text { zu grob } & \text { nicht } \\ 0 & 0 & 0 & 0 & 0\end{array}$


[]Die vorherige Frage trifft nicht zu, weil *

Beantworten Sie diese Frage mut, wonn folgende Bedingungen erfult sind:

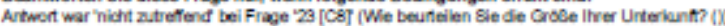

Bane geben Bie lhre Avtwort hier ein

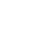

L

[]Welche Möglichkeit gab es, in der Unterkunft zu kochen? * Bae whilen Ble de zatreffende Antwort tar josten Punkt as: geringe Moglichike

(z.B.

Mkrowele, normal
keine Wrobzogla

Massenocher, suagestattete ausgestatete nicht
Kocheilt

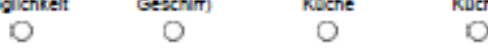

[]Die vorherige Frage trifft nicht $\mathbf{z u}$, weil *

Beantworten Sie diese Frage mus, wonn folgende Bedingungen erfult sind:

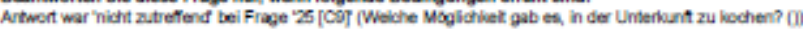

Bne geben Bie hre Antwort hier ein.

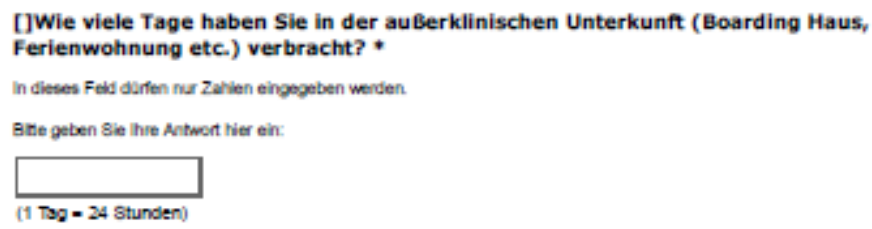

[JWie viele Tage haben Sie in der auBerklinischen Unterkunft (Boarding Haus, Ferienwohnung etc.) verbracht? *

In dieses Feld diffen nur Zavien eingogeben werden.

Bane geben Bie thre Antwort hier ein.

(1 T9g - 24 Stunden) 


\section{Geburt}

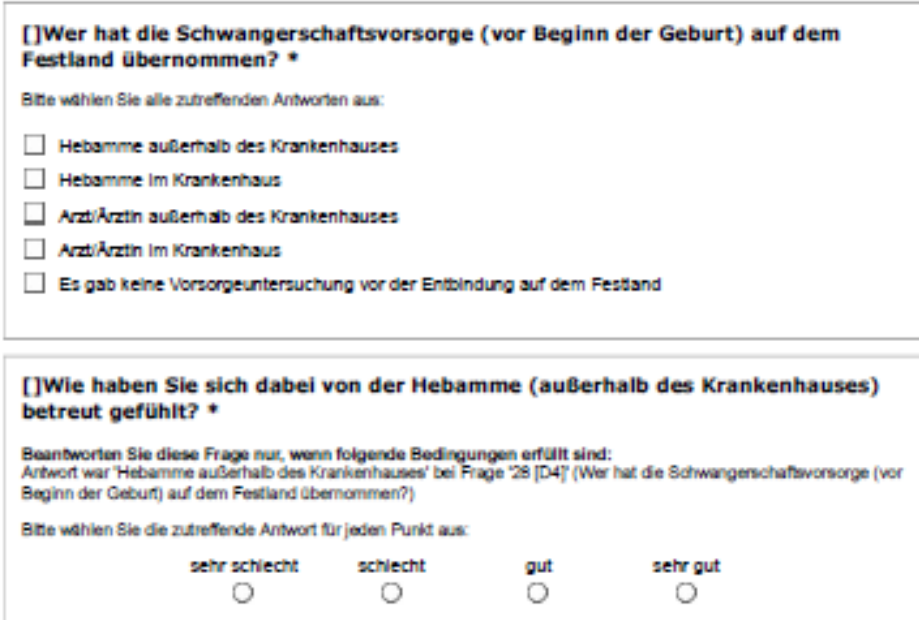

[]Wie haben Sie sich dabei von der Hebamme im Krankenhaus betreut gefühit? * Beantworten Sie diese Frage nu, wonn folgende Bedingungen erfult sind:

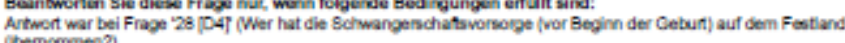
abemommen?

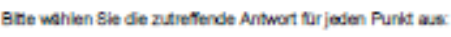

$\begin{array}{cccc}\text { serr schiecht } & \text { schiecht } & \text { gut } & \text { sehr gut } \\ Q & Q & Q & Q\end{array}$

[]Wie haben Sie sich dabei von dem Arzt/ der Ärztin (auberhalb des Krankenhauses) betreut gefühit? *

Beantworten Sie diese Frage nus, wonn folgende Bedingungen erfult sind:

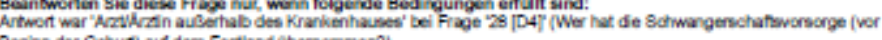
Beginn der Cobunt) ald dem Festland abenommen?

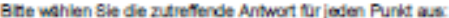

$\begin{array}{cccc}\text { serr schiecht } & \text { schiecht } & \text { gut } & \text { sehr gut } \\ 0 & 0 & 0 & 0\end{array}$


[]Wie haben Sie sich dabei von dem Arzt/ der Ärztin im Krankenhaus betreut gefuhit? *

Beantworten Sie diese Frage nut, wenn folgende Bedirgungen erfult sind:

A cbemommen?

Bne wathien Sie die zutrefende Antwort tur jocien Punkt as:
senr schiect
echischt
gut sehr gut

(

O

0

[]Von wie vielen Hebammen wurden Sie wahrend der Geburt betreut? *

in dieses Feld dirften nur Zavien eingogeben werden.

Bate geben 8 ie thre Antwort hier en:

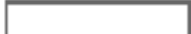

[]Wie war die Hebamme für Sie wăhrend der Geburt ansprechbar? *

Bne wahien Sie die zutreftende Antwort fur joblen Punkt as:

$\begin{array}{ccccc}\text { serr } & & & \text { nicht } \\ \text { schiecht } & \text { schiecht } & \text { gut } & \text { sehr gut } & \text { zutreftend } \\ 0 & 0 & 0 & 0 & 0\end{array}$

[DDie vorherige Frage trifft nicht $\mathrm{zu}$, weil *

Beantworten Sie diese Frage mus, wonn folgende Bedingungen eff Uitt sind:

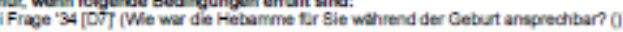

Bne geben 8ie thre Antwont tier ein

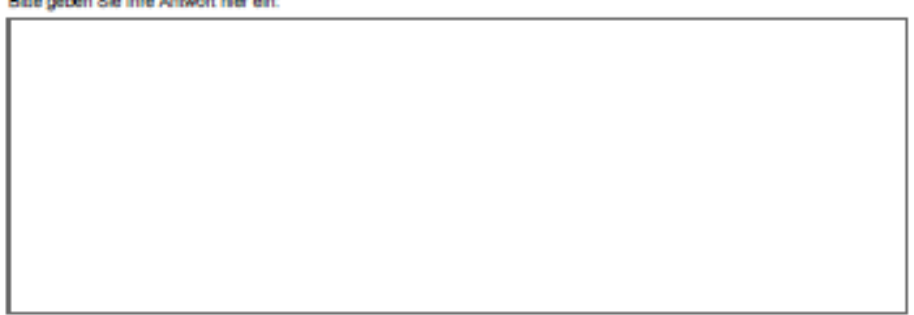

[QVon wievielen Ärztinnen und Ärzten wurden Sie wăhrend der Geburt betreut? * In dieses Feld dirfen nur Zavien eingogeben werden.

Bne geben 8ie hre Antwort hier ein: 
[]Wie war der Arzt/ die Ärztin bzw. waren die Ärzte für Sie wăhrend der Geburt ansprechbar?

Beantworten Sie diese Frage nue, wonn folgende Bed gungen erfult sind

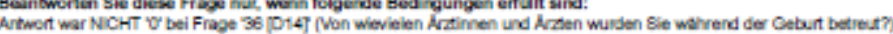

Bne whilen Bie die zitrefende Antwort fur jodien Purbt as:

$\begin{array}{ccccc}\begin{array}{c}\text { serr } \\ \text { schiecht }\end{array} & \text { schiecht } & \text { gut } & \text { sehr gut } & \text { nicht } \\ 10 & 0 & 0 & 0 & 0\end{array}$

[]Die vorherige Frage trifft nicht zu, weil *

Gantworten Sie diese Frage nut, woma folgende Bedingungen erfuit sint

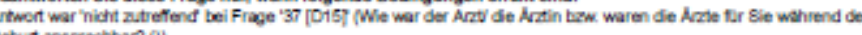

The geben 8 ie thre Actroct wive ein

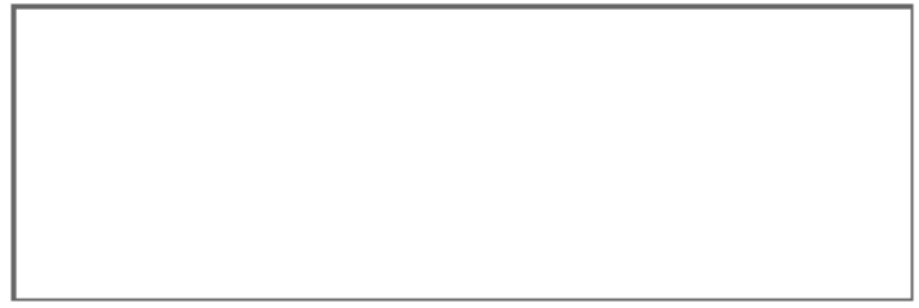

[]Geburtsmodus *

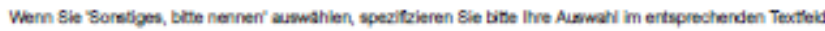

Bate wahien Sie nur aine der folgerden Avowoten ais

Spontan

Kalserschnitt

Sougglocke

sonstiges, bitte nennen

[]Hatten Sie eine Geburtseinleitung? *

Bne watien Sle nur edine der folgenden Avowoten as:

斻

Nen 
[]Aus welchem Grund wurde die Geburtseinleitung vorgenommen? *

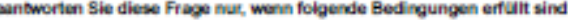

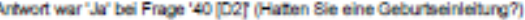

Bate geben sie he Avtwot hier ein:

Thas

[]Brauchte Ihr Kind nach der Entbindung eine weiterfuhrende Betreuung in der Kinderklinik? *

ate wahien Sie nur aine der folgenden Avtwoten aus

0 da

Nen

[]Warum brauchte Ihr Kind eine weiterfuhrende Betreuung in der Kinderklinik? * Beantworten Sie diese Frage mus, wom folgende Bedingungen erfult sind:

(a) (a)diniv?

ane geben 8ie thre Antwort hier en:

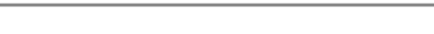

[]Wie haben Sie sich nach der Geburt auf der Wochenstation insgesamt betreut gefuhit? *

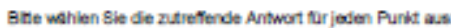

$\begin{array}{lccccc} & \begin{array}{c}\text { secr } \\ \text { schilecht }\end{array} & \text { schilecht } & \text { gut } & \text { sehr gut } & \begin{array}{c}\text { nicht } \\ \text { zutreffend }\end{array} \\ \text { Von den Avaten } & 0 & 0 & 0 & 0 & 0 \\ \text { Von den Hebammen } & 0 & 0 & 0 & 0 & 0 \\ \text { Von dem } & 0 & 0 & 0 & 0 & 0 \\ \text { Pliegeperosnal } & 0 & 0 & & \end{array}$

[]Die vorherige Frage trifft nicht zu (Betreuung durch Ärzte auf der Wochenstation), weil *

Beantworten Sie diese Frage mut, wenn folgende Bedingungen erfult sind:

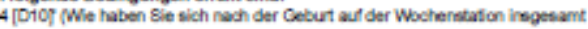
verreit gefinh

Bne geben Be thre Antwort hier ein:

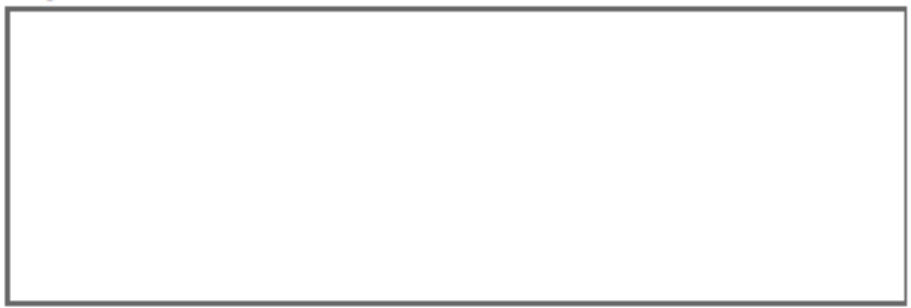

[]Die vorherige Frage trifft nicht zu (Betreuung durch Hebammen auf der Wochenstation), weil

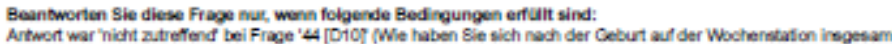
betreut gefunit? (ven den Hebammeny)

Bne geben 8 ie thre Avtront hier ein: 
[]Die vorherige Frage trifft nicht zu (Betreuung durch Pflegepersonal auf der Wochenstation), weil *

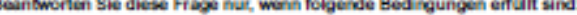

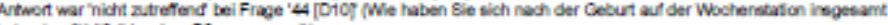
betreut gefunin? (von dem Plogepersonali)i

Bue geben Bie thre Antwort wier ein:

[]Wieviele Tage sind Sie nach der Geburt noch in der Entbindungsklinik gewesen? In dieses Feld diffen nua Zakien eingegeben werder

Bne geben Bie hive Avtwort hier ein.

(1 T9g -24 Stunden)

[]Wieviele Tage waren Sie ingesamt in der Entbindungsklinik? *

In dieses Fedd dirfen nua Zavien eingegeben werder

Bne geben 8ie lhre Avtmont hier ein.

(1 Tog -24 Stunden) 


\section{Transport}

[]Mit weichem Transportmittel sind Sie zum Geburtsort gekommen? (Mehrfachnennung möglich) *

Bne watien Bie alle zutrefenden Antworten aus:

$\square$ Anto

$\square$ Fanre

$\square$ Hubactrauber

$\square$ seenotettungekreuzer

$\square$ Rettungewapen

$\square$ Andere, bltie nernen:

[]Mit welchem Transportmittel sind Sie vom Geburtsort wieder nach Hause gekommen? (Mehrfachnennung mb̈glich) *

Bre wahien Sie alle zidreffenden Antworten aus

$\square$ Auto

$\square$ Fathre

$\square$ Zug

$\square$ Bue

$\square$ Andere, bitte nernen:

[]Bitte geben Sie stichwortartig an, welche Angehörige, Freunde, Bekannte oder andere Personengruppen bel der Organisation rund um die Geburt involviert waren (z.B. Mutter, Vater, Ehemann, Nachbar etc.). Wenn es zum Beispiei mehrere verschiedene Freunde/Bekannte waren, kennzeichnen Sie dies bitte mit Freund/Bekannter 1, Freund/Bekannter 2 etc.)

Bezogleh des Transports auf dys Fegtand und zurock nach For Bez0glch der Begletung vor Ort (z. B. durch Parther, Ettem etc) Bez0gich der KInderoetreuung tor mogliche Gegchwiaterkinder Bezuglich der Versorgung von anderen Familenmitgledem, de Sie sonet versorgen

Bezogleh der Veroorgung von WohnungHsus

Bezuglich der Veroorgung von Hauatieren

Bezogich anderer Tstigketen

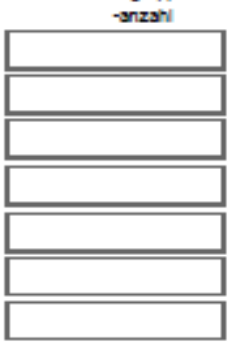

[]Bitte nennen Sie die "anderen Tatigkeiten". *

Beantworten Sie diese Frage mul, wonh folgende Bedingungen enfult sine:

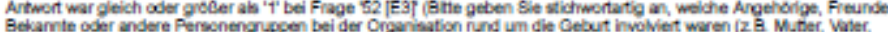

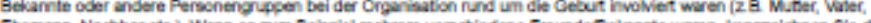

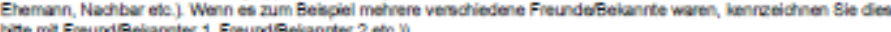

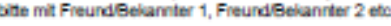

Bne geben 8ie thre Avtront tier ein: 


\section{Kosten}

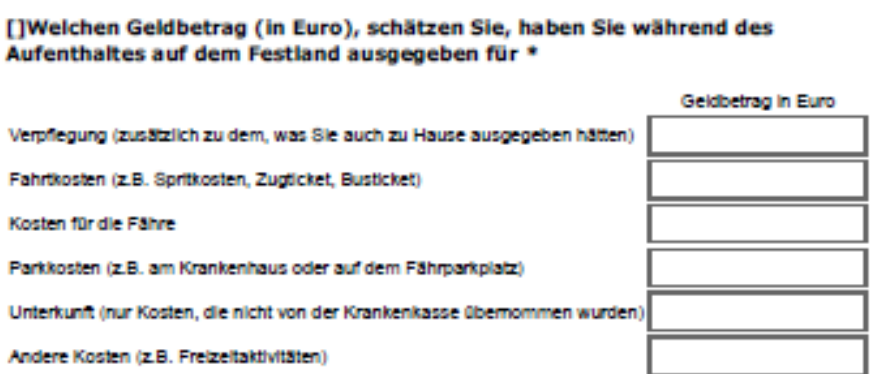

[]Wofür genau waren die "anderen Kosten"? *

Baentworten Sie diese Frage nus, wonn folgende Bedingungen erfult sind:

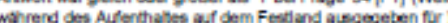

Gate geben Bie lhe Avtwort hier ein

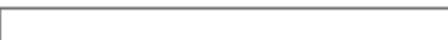

[]Hatten Sie wăhrend des Aufenthalts auf dem Festland zur Geburt einen Verdienstausfall bei z.B. einer Selbständigkeit? *

Bne wahien Sie nur eine der folgerden Aveworten aus

$O$ da

Nen

[]Wie hoch ca. war Ihr Verdienstausfall? *

Baantworten Sie diese Frage mul, worn folgende Bed igungen erfult sind:

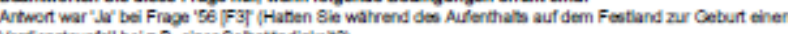
Verdenstasatal bei 28 . einer Belbotindigint?

In clieses Fedd dirften nu Zarien eingogeben wenden

Gne geben 8ie thre Antwort hier ent.

Euro

[]Hatte Ihr Partner wathrend des Aufenthalts auf dem Festland zur Geburt einen Verdienstausfall z. B. bei einer Selbststăndigkeit? *

Bate wahien Sie nur dine der folgenden Avowoten as

0 sa

Nein

\section{[]Wie hoch ca. war der Verdienstausfall Ihres Partners?}

Beantworten Sie diese Frage nue, wern folgende Bedingungen erfult sind

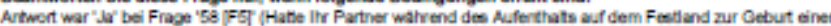

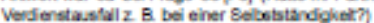

In dieses Fedd dirfen nur Zahien ehopgeben werden.

Bne geben Sie hre Antwort tier ein:

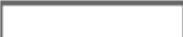

Euro 


\section{Perspektive}

[ Nehmen Sie an, Sie planten noch ein Kind zu bekommen, wo würden Sie Ihr nächstes Kind bekommen wollen? *

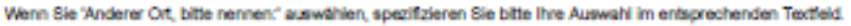

Bate whilen Bie nur dine der folgenden Avowoten aus:

Hauageburt

Geburtehsus aut der inze:

Krankenhaus ohne Knderkinik ( $z$. B. Hueum)

Krankenhaua ma Kindenkinik (z.B. Flenaburg, Helde)

Anderer Ort, bltte nernen:

[]In diesem letzten Abschnitt finden Sie Aussagen zur Geburtshilfe auf Fohr bzw. auf dem Festland. Bitte geben Sie an, wie stark die einzelnen Aussagen auf Sie zutreffen. *

Bne whilen Ble die zutrefende Antwort fur josten Punkt as:

In der schwangerschat

habe ich mich nicht auf das

Kind getreut, well lch mir

Gedanken ober de

verbnderten Umatsinde run

um de Geburt gemach:

Wern Ich Jeat noch ein Kind

haben wolise, worste Ich,

wie leh die Geburt
organialeren aol.

Es lat eine Belostung for

gebortlges "Inselkind" let.

Die Organization nund um

die Geburt war eine

psychische Belastung for de

ganze Famlle.

Ich bin in der KInk auf dem

Festand in meiner Peroon

Die Entremung zu meiner

Famile durch den Ausentho:

ouf dem Featind zur Gebu

Furmich lch als besstrot

Fur mich spielt de

sichentet (z.s.

angeschiosaene

Geburt eine grobe Role.

Meine Famllenolanung lat

autorund de:

geburtahifichen stustion

out der insel abgeachlogren.

Es musa kiarer gerege:

werden, wer fur de

Vorsorge und

schwangerschansbegletung

ouf dem Featand bls zum

Beginn der Geburt

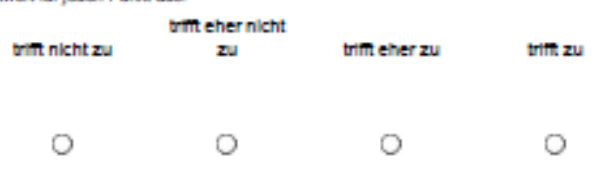

$\begin{array}{llll}0 & 0 & 0 & 0\end{array}$

verantwortich lat. 
[]Haben Sie über das Kind hinaus, über dessen Geburt Sie in dieser Umfrage berichtet haben, im Zeitraum vom 01. Oktober 2015 bis zum 30. September 2017 noch weitere Kinder bekommen?

Gine whilien Ble nur eine der folgenden Avoworten as.

Q va

Nen

[]Wie viele weitere Kinder haben Sie noch bekommen? *

Beanoworten Sie diese Frage mus, wonn folgende Bedingungen enfult sind:

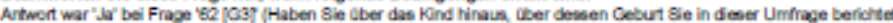
heten, im Zetra.m voen 01. Olsober 2015 bis zum 30 . 8eptember 2017 noch welhere Kinder bekommen?

In dieses Feld dirfen nu Zavien eingogeben werden

Bne geben 8ie thre Antwort tier ein

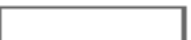

[] Jetzt sind Sie am Ende des Fragebogens angelangt, vielen Dank. Waren Fragen

missverständlich oder aus Ihrer Sicht nicht beantwortbar- dann lassen Sie es uns an dieser Stelle wissen. Auch auf weitere Kommentare freuen wir uns.

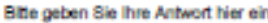


Google Maps Dagebüll nach Diakonissenkrankenhaus Mit dem Auto 56,8 km, 58 Min. Flensburg

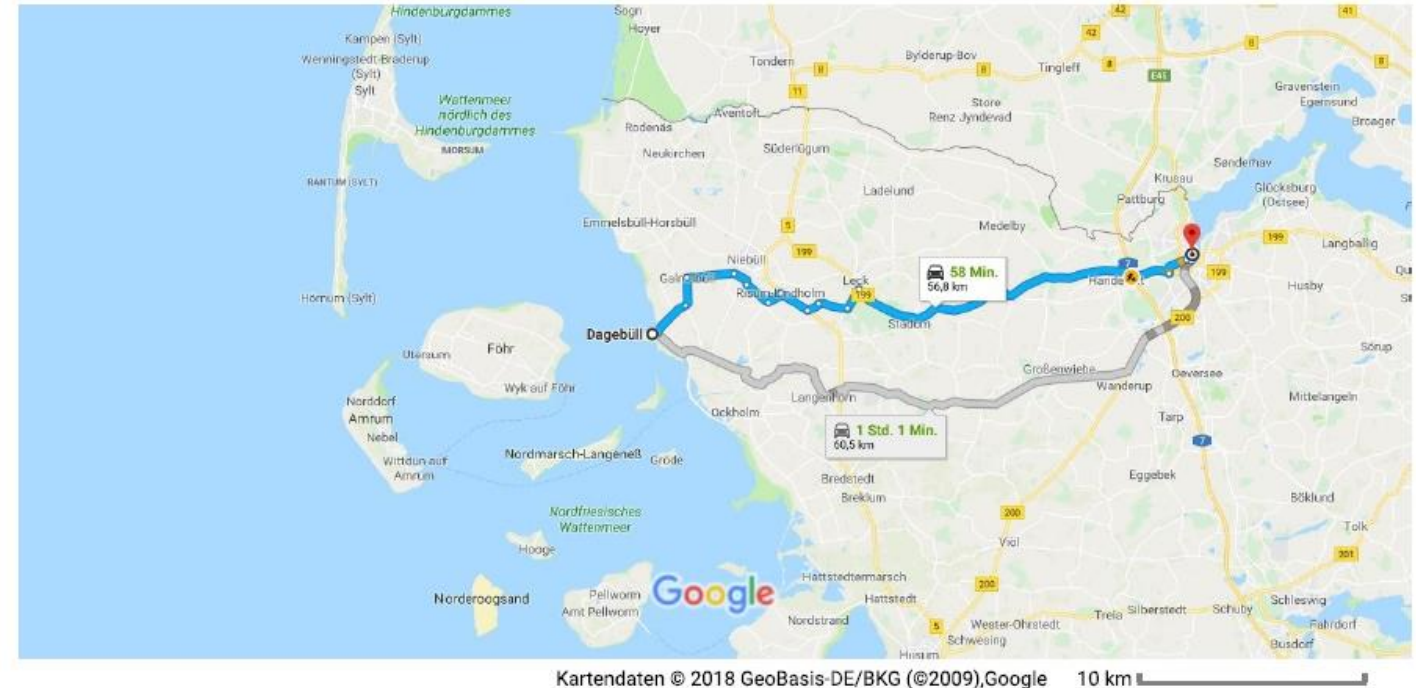

\section{$\begin{array}{ll}\text { Google Maps } & \begin{array}{l}\text { Dagebüll nach WKK Heide } \\ \text { Westküstenklinikum Heide }\end{array}\end{array}$}

Mit dem Auto 84,7 km, 1 Std. $32 \mathrm{Min}$.

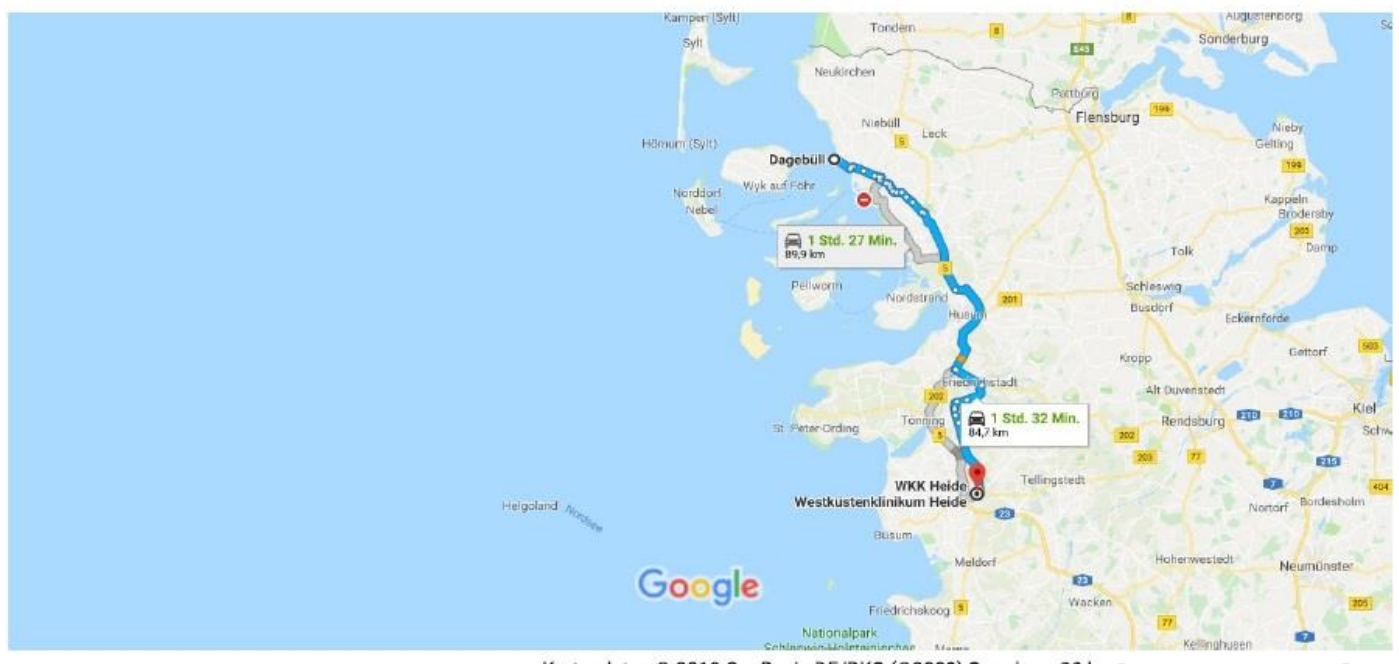

Kartendaten (๑) 2018 GeoBasis-DE/BKG (๑2009),Google

$20 \mathrm{~km}$ 


\begin{tabular}{|c|c|c|c|c|c|}
\hline Auskunft erfragt über & Kontakt & $\begin{array}{l}\text { Art des } \\
\text { Kontaktes }\end{array}$ & $\begin{array}{l}\text { Zeitpunkt } \\
\text { des } \\
\text { Kontakts }\end{array}$ & $\begin{array}{l}\text { Antwort } \\
\text { erhalten }\end{array}$ & $\begin{array}{l}\text { Auskunft } \\
\text { erhalten }\end{array}$ \\
\hline $\begin{array}{l}\text { Rettungssystem } \\
\text { Schleswig-Holstein }\end{array}$ & $\begin{array}{l}\text { Herr L., Kreis } \\
\text { Nordfriesland }\end{array}$ & E-Mail & 14.03 .2018 & $\mathrm{Ja}$ & $\mathrm{Ja}$ \\
\hline DRF Luftrettung & Station Niebüll & $\begin{array}{l}\text { E-Mail und } \\
\text { telefonisch }\end{array}$ & $\begin{array}{l}21.03 .2018 \\
\text { und } 16.04 . \\
2018\end{array}$ & $\mathrm{Ja}$ & Nein \\
\hline DGzRS & M. K. & Telefonat & 06.04 .2018 & $\mathrm{Ja}$ & $\mathrm{Ja}$ \\
\hline Boarding Flensburg & Pressestelle & Telefonat & 19.04 .2018 & $\mathrm{Ja}$ & Nein \\
\hline Boarding Flensburg & Haus Pniel & E-Mail & 12.04 .2018 & Nein & Nein \\
\hline Boarding Heide & $\begin{array}{l}\text { Sekretärin } \\
\text { Chefarzt }\end{array}$ & E-Mail & 16.04 .2018 & Nein & Nein \\
\hline Boarding & AOK Nordwest & E-Mail & 03.04 .2018 & $\mathrm{Ja}$ & Nein \\
\hline Boarding & BKK Nordwest & E-Mail & 03.04 .2018 & Nein & Nein \\
\hline Boarding & IKK Nord & E-Mail & 03.04 .2018 & Nein & Nein \\
\hline Boarding & vdek & Telefonat & 19.04.2018 & $\mathrm{Ja}$ & $\mathrm{Ja}$ \\
\hline Boarding & $\begin{array}{l}\text { TK Schleswig- } \\
\text { Holstein }\end{array}$ & E-Mail & 20.04.2018 & $\mathrm{Ja}$ & $\mathrm{Ja}$ \\
\hline Boarding & $\begin{array}{l}\text { DAK Schleswig- } \\
\text { Holstein }\end{array}$ & $\begin{array}{l}\text { E-Mail und } \\
\text { Telefonat }\end{array}$ & 20.04 .2018 & $\mathrm{Ja}$ & Nein \\
\hline Gutachten & FDP Nordfriesland & E-Mail & 16.04 .2018 & $\mathrm{Ja}$ & $\mathrm{Ja}$ \\
\hline Gutachten & $\begin{array}{l}\text { Kreis } \\
\text { Nordfriesland }\end{array}$ & E-Mail & 20.04 .2018 & $\mathrm{Ja}$ & $\mathrm{Ja}$ \\
\hline Gutachten & $\begin{array}{l}\text { Krankenhaus } \\
\text { Nordfriesland }\end{array}$ & E-Mail & 20.04 .2018 & Nein & Nein \\
\hline Beleggeburt & $\begin{array}{l}\text { Gynäkologin auf } \\
\text { Föhr }\end{array}$ & $\begin{array}{l}\text { E-Mail }(2 x) \\
\text { und } \\
\text { telefonisch } \\
(2 x)\end{array}$ & $\begin{array}{l}\text { Dezember } \\
2017 \text { und } \\
\text { März } 2018\end{array}$ & Nein & Nein \\
\hline Beleggeburt & $\begin{array}{l}\text { Landesvorsitzende } \\
\text { Berufsverband der } \\
\text { Frauenärzte e.V. }\end{array}$ & Telefonat & 02.05 .2018 & $\mathrm{Ja}$ & $\mathrm{Ja}$ \\
\hline Hebammennotruf & $\begin{array}{l}\text { Kreis } \\
\text { Nordfriesland, } \\
\text { Fachbereich } \\
\text { Jugend, Familie } \\
\text { und Bildung }\end{array}$ & $\begin{array}{l}\text { E-Mail und } \\
\text { Telefonat }\end{array}$ & 07.05 .2018 & $\mathrm{Ja}$ & $\mathrm{Ja}$ \\
\hline $\begin{array}{l}\text { Haftpflichtversicherung } \\
\text { Gynäkologin und } \\
\text { Hebammen }\end{array}$ & $\begin{array}{l}\text { Geschäftsführer } \\
\text { Krankenhaus } \\
\text { Nordfriesland }\end{array}$ & $\begin{array}{l}\text { E-Mail und } \\
\text { Telefonat }\end{array}$ & 24.08 .2018 & $\mathrm{Ja}$ & $\mathrm{Ja}$ \\
\hline
\end{tabular}




\author{
MEDIZINISCHE FAKULTÄT \\ DER CHRISTIAN-ALBRECHTS-UNIVERSITÄT ZU KIEL
}

ETHIK-KOMMISSION

Universitäts-Kinderklinik · Schwanenweg 20 · 24105 Kiel

Prof. Dr. med. Hanna Kaduszkiewicz Institut für Allgemeinmedizin

UKSH Campus Kiel

Arnold-Heller-Straße 3, Haus 17

$24105 \mathrm{Kiel}$

\section{AZ.: \\ Studienplan:}

Antrag vom:
D 564/17 (bitte stets angeben)

Geburtserfahrungen von Insulanerinnen nach Schließung der stationären Geburtshilfe auf Föhr und

Direkte und indirekte Kosten einer Geburt bei Insulanerinnen, wenn vor Ort keine Geburtshilfe möglich ist

Anschreiben v. 13.11.17; Basisformular für einen Ethik-Antrag v. 13.11.17; Informationsblatt für Studienteilnehmer V. 1.0; Online Umfragebogen V. 1.0 Studienprotokoll V. 1.0;

Prof. Dr. Hanna Kaduszkiewicz, UKSH Campus Kiel

Doktorandinnen Katja Baudisch, Jill Charlotte Jaursch

13. November 2017 (Eingang: 14. November 2017)

Sehr geehrte Frau Kollegin Kaduszkiewicz,

wir bestätigen den Eingang des obengenannten Antrages zur Beratung gemäß § 15 Berufsordnung (BO) der Ärztekammer Schleswig-Holstein.

Nach Durchsicht der Unterlagen durch die Geschäftsstelle und durch mich als Vorsitzenden der Ethik-Kommission bestehen gegen die Durchführung der Studie keine berufsethischen und berufsrechtlichen Bedenken.

Die im Folgenden aufgeführten Hinweise müssen beachtet werden:

1. Es wird darauf hingewiesen, dass künftige Änderungen der Studie der Ethik-Kommission anzuzeigen sind und gegebenenfalls eine erneute Beratung erforderlich machen.

2. Die ethische und rechtliche Verantwortung für die Durchführung dieser Studie verbleibt beim Studienleiter.

3. Die Ethik-Kommission weist darauf hin, dass für eventuell in Zukunft weitere teilnehmende Zentren eine berufsrechtliche Beratung bei der jeweils für sie zuständigen Ethik-Kommission erforderlich ist.

4. Gemäß Deklaration von Helsinki muss der Ethik-Kommission nach Studienende ein Abschlussbericht vorgelegt werden, der eine Zusammenfassung der Ergebnisse und Schlussfolgerungen der Studie enthält.

Wir wünschen Ihnen für die Durchführung der Studie viel Erfolg.

Mit freundlichen kollegialen Grüßen

Prof. Dr. med. H. M. Mend

Vorsitzender der Ethik-Kommission

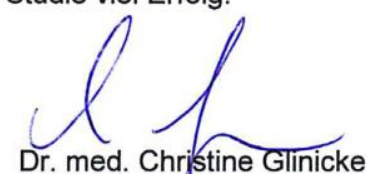

Geschäftsführung der Ethik-Kommission 


\section{1 A $1 \mathbf{U} \quad$ Medizinische Fakultät \\ Christian-Albrechts-Universität zu Kiel

Direktorin:

Prof. Dr. med. Hanna Kaduszkiewicz

Michaelisstr. 5, $24105 \mathrm{Kiel}$

$\begin{array}{ll}\text { Sekretariat } & \text { Birgit Blunck } \\ & \text { office@allgemeinmedizin.uni-kiel.de } \\ & +49431500-30101 \text { (Tel.) } \\ +49 & 431500-30104 \text { (Fax.) }\end{array}$

\section{Forschungsvorhaben „Geburtserfahrungen und -kosten von Insulanerinnen nach Schließung der stationären Geburtshilfe auf Föhr"}

\section{Einverständniserklärung}

Ich wurde über die oben genannte wissenschaftliche Untersuchung ausreichend schriftlich und mündlich informiert und hatte die Möglichkeit, Fragen zu stellen.

Ich bin damit einverstanden, dass die im Rahmen der wissenschaftlichen Untersuchung erhobenen Daten sowie meine sonstigen mit dieser Untersuchung zusammenhängenden personenbezogenen Daten elektronisch und in Papierform aufgezeichnet werden. Das persönliche Interview wird auf einen Datenträger aufgezeichnet, wörtlich transkribiert, anonymisiert und wissenschaftlich ausgewertet. Es wird gewährleistet, dass meine personenbezogenen Daten nicht an Dritte weitergegeben werden. Bei der Veröffentlichung in einer wissenschaftlichen Zeitschrift wird aus den Daten nicht hervorgehen, wer an dieser Untersuchung teilgenommen hat.

Mit meiner Unterschrift erkläre ich, dass ich mit vorstehend geschilderter Vorgehensweise einverstanden bin und ich zustimme, an diesem Projekt teilzunehmen.

Meine Teilnahme an der Studie ist freiwillig. Wenn ich nicht teilnehme, werde ich keine Nachteile haben.

Ich weiß, dass ich diese Zustimmung jederzeit und ohne Angabe von Gründen widerrufen kann, ohne dass für mich Nachteile entstehen. 


\section{Danksagung}

An dieser Stelle möchte ich mich bei einigen Personen bedanken, die mich bei der Erstellung der Arbeit über die Jahre hinweg begleitet und unterstützt haben.

Vielen Dank an meinen Doktorvater, Herrn Prof. Dr. Diel, für die unglaublich unkomplizierte und kompetente Betreuung. Danke, dass Sie zu jederzeit erreichbar waren und mir immer das Gefühl gegeben haben, dass wir gemeinsam dieses Projekt erfolgreich beenden werden.

Danke an Frau Prof. Dr. Kaduszkiewicz für die Initiierung der Arbeit und die Betreuung während der ersten Phase der Datenerhebung auf der Insel Föhr. Danke auch für die Hilfe im weiteren Verlauf der Erstellung der Arbeit.

Ein großes Dankeschön an Herrn Hinrichsen vom Verein Inselgeburt für das freundliche Willkommen auf der Insel Föhr und die Herstellung des Kontaktes zu den Teilnehmerinnen der Studie. Vielen Dank an alle Teilnehmerinnen der Studie, die sich Zeit genommen haben für die Interviews und/ oder das Ausfüllen des Fragebogens.

Liebe Katja, danke für die unkomplizierte und effiziente Zusammenarbeit während der ersten Phase dieser Arbeit.

Lieber Lorenz, danke, dass du an meiner Seite bist und mich selbstverständlich unterstützt.

Liebe Kornelia, lieber Julian, danke für eure großartige Unterstützung und eure hilfreichen Ratschläge zu jeder Zeit. Danke, dass ich euch habe, mit euch diskutieren kann und ihr mein Rückhalt in jeder Lebenslage seid. 


\section{Publikation}

Jaursch C, Kaduszkiewicz H, Diel R. Direkte und indirekte Kosten einer Geburt bei Insulanerinnen, wenn vor Ort keine Geburtshilfe möglich ist: Georg Thieme Verlag KG; 2019 